\title{
Summaries of FY 1996 Engineering Research
}

June 1997

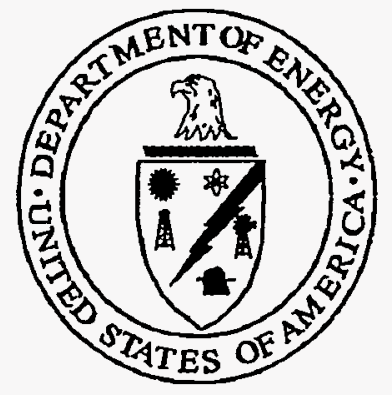

U.S. Department of Energy

Office of Energy Research

DUSTRIBUTION OF THIS DOCUMENT IS UNLIMITED Office of Basic Energy Sciences Division of Engineering and Geosciences Germantown, MD 20874 


\section{DISCLAIMER}

Portions of this document may be illegible in electronic image products. Images are produced from the best available original document. 


\section{Foreword}

This report documents the Basic Energy Sciences (BES) Engineering Research Program for fiscal year 1996; it provides a summary for each of the program projects in addition to a brief program overview. The report is intended to provide staff of Congressional committees, other executive departments, and other DOE offices with substantive program information so as to facilitate governmental overview and coordination of Federal research programs. Of equal importance, its availability facilitates communication of program information to interested research engineers and scientists. Each BES Division administers basic, mission oriented research programs in the area indicated by its title. The BES Engineering Research Program is one such program; it is administered by the Engineering and Geosciences Division of BES. Dr. Robert E. Price is technical manager of the Engineering Research Program; inquiries concerning the program may be addressed to him, in writing, by phone at (301) 903-3565 or by fax at (301) 903-0271 (additional information and updates are accessible on World Wide Web, http://er.doe.gov).

In preparing this report we asked the principal investigators to submit summaries for their projects that were specifically applicable to fiscal year 1996. The summaries received have been edited if necessary, but the press for timely publication made it impractical to have the investigators review and approve the revised summaries prior to publication. For more information about a given project, it is suggested that the investigators be contacted directly 


\section{Introduction}

The individual project summaries follow the program overview. The summaries are ordered alphabetically by name of institution; the table of contents lists all the institutions at which projects were sponsored in fiscal year 1996.

Each project entry begins with an institutional-departmental heading. The names of investigators are listed immediately below the title. The funding level for fiscal year 1996 appears to the right of title; it is followed by the budget activity number (e.g., 01-A). These numbers categorize the projects for budgetary purposes and the categories are described in the budget number index. A separate index of Principal Investigators includes phone number, fax number and e-mail address, where available. The fiscal year in which either the project began or was renewed and the anticipated duration in years are indicated respectively by the first two and last digits of the sequence directly below the budget activity number (e.g., 94-3). The summary description of the project completes the entry 


\section{Program Review BES Engineering Research}

The BES Engineering Research Program is one of the component research programs which collectively constitute the DOE Basic Energy Sciences Program. The DOE Basic Energy Sciences program supports energy related research in the physical and biological sciences, and in engineering. The chief purpose of the DOE Basic Energy Sciences Program is to provide the fundamental scientific base on which-identification-and-development-of-future,-national-energy-options-will-dependThe major product of the program becomes part of the body of data and knowledge upon which the applied energy technologies are founded; the product is knowledge relevant to energy exploration, production, conversion and use.

The BES Engineering Research Program was started in 1979 to help resolve the numerous serious engineering issues arising from efforts to meet U.S. energy needs. The program supports fundamental research on broad, generic topics in energy related engineering topics not as narrowly scoped as those addressed by the shorter term engineering research projects sponsored by the various $D O E$ technology programs. Special emphasis is placed on projects which, if successfully concluded, will benefit more than one energy technology. During the first year several workshops were sponsored for the purpose of identifying energy related engineering research needs and initial priorities. Representatives from industry, academic institutions, national laboratories, and leading members of professional organizations (Engineering Societies Commission of Energy, American Society of Mechanical Engineers, Society of Automotive Engineers, and Joint Automation and Control Committee) participated in the workshops. In addition to the participants in the workshops, staff representatives from the DOE technology programs and other leading U.S. energy engineering experts made significant contributions to the setting of program priorities. There resulted from this process a strong confirmation of the need for a long range, fundamental engineering research program with two major goals. The broad goals that were established by this process for the BES Engineering Research Program are:

1) To extend the body of knowledge underlying current engineering practice so as to create new options for enhancing energy savings and production, for prolonging useful equipment life, and for reducing costs without degradation of industrial production and performance quality; and

2) To broaden the technical and conceptual base for solving future engineering problems in the energy technologies. 


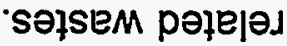

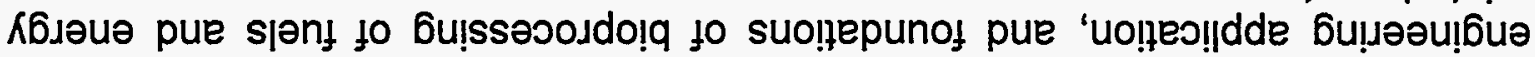

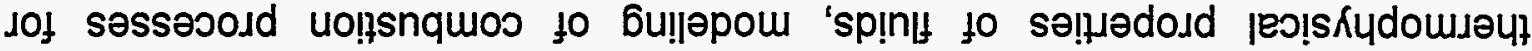

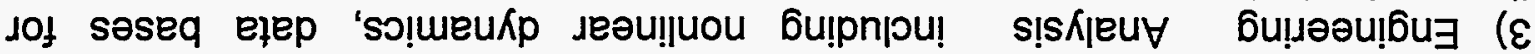

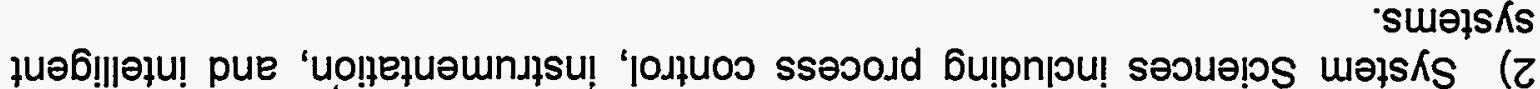

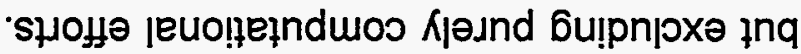

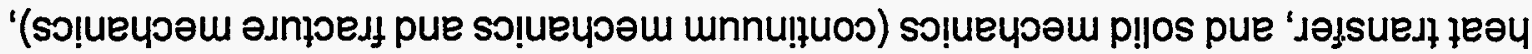

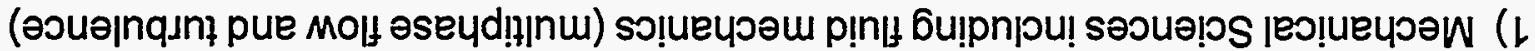

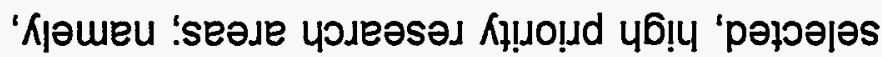

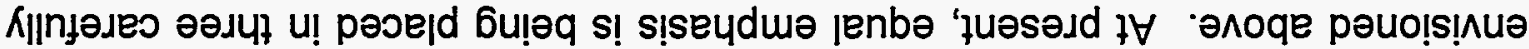

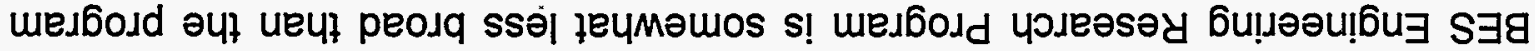

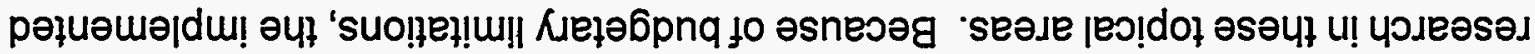

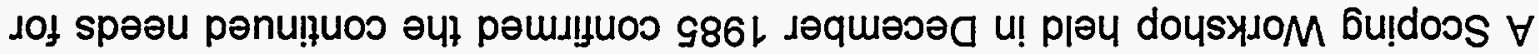

słu!̣edisuos umoux of łoə!̣ns

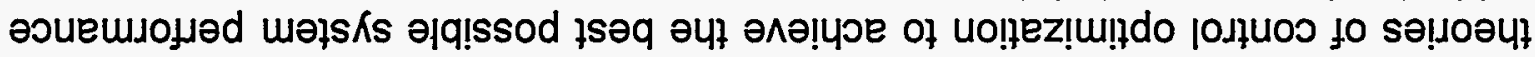

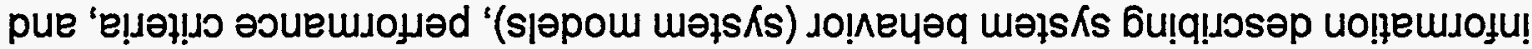

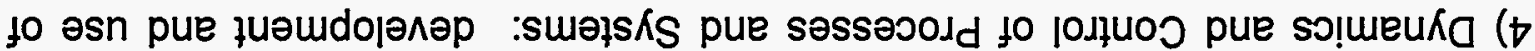

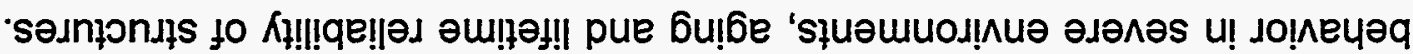

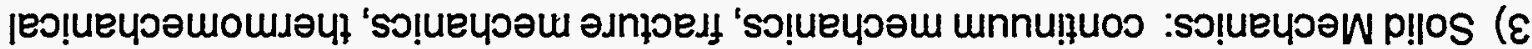

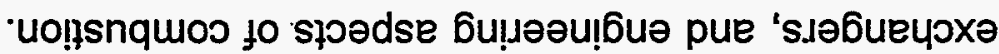

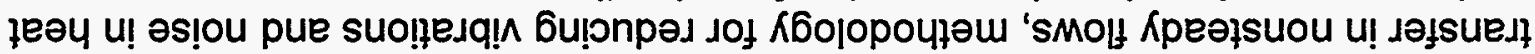



suəịsks

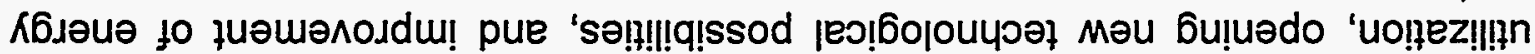

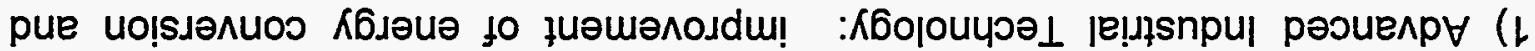

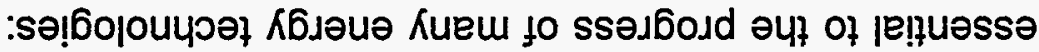

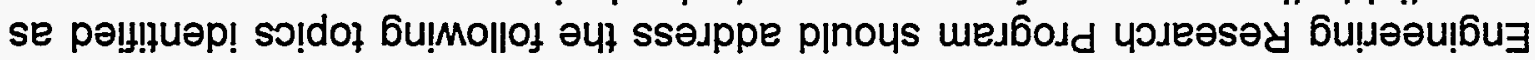

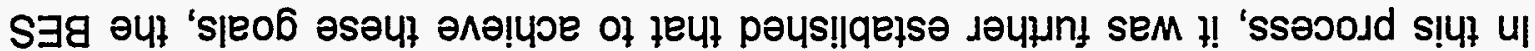


These areas contain the most critical elements of the four topics enumerated above; as such they are of importance to energy technologies both in the short and long term, and therefore of immediate programmatic interest. It should be noted that other areas of basic research important to engineering are monitored elsewhere in BES. For instance, separation sciences and research on thermophysical properties are among the responsibilities of the Chemical Sciences Division, while microscopic aspects of fracture mechanics are in the domain of the Material Sciences Division. As resources permit, other high priority areas are being added to the Engineering Research Program. Thus, as a result of previous growth in the program budget an important development took place in the Engineering Research Program: two major concentrations of research were initiated.

First, a new program was organized at Oak Ridge National Laboratory dealing with intelligent machines in an unstructured environment. Some resources are available for coordinated, more narrowly focussed, related, high quality research at universities and other research centers. All such activities are supported and administered directly by the Engineering Research Program, but some coordination of efforts with the ORNL program may prove useful.

Secondly in FY 1985, a collaborative research effort was started between MIT and Idaho National Engineering Laboratory. At present, the collaboration is in two distinct-areas:-Automated-Welding-and-Fracture Mechanics-Collateral,-high-quality research efforts at other institutions, including Plasma Process Engineering are supported by the Engineering Research Program.

In the expectation of a future modest growth of this Program, three International Workshops on Two Phase Flow Fundamental were held one in September 1985 and the other in March 1987. The meetings were used to identify basic research needs in the field of two phase flow and heat transfer; summary reports of the workshops are available from the Program Office. The proceedings of the two workshops have been published as volumes in the series "Advances in Heat and Mass Transfer" (Hemisphere Publishing Company). A third international workshop held in June 1992 surveyed the status of the field. The proceedings have been published by CRC Publishing Company, also in the series "Advances in Heat and Mass Transfer"

Two additional workshops were held during 1988 . The first dealt with possible research opportunities in the field of novel devices using the new high temperature superconductors. The second addressed research needs for bioprocessing of fuels and energy related wastes. Reports of both workshops have been published. Additional funds had been provided in FY 1992 to initiate research in the above mentioned bioprocessing area. Of interest are relevant studies at the intersection of biology, biochemistry, and chemical engineering. 


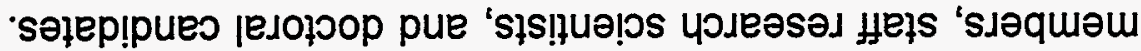

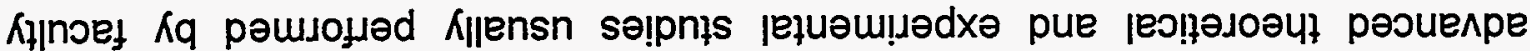

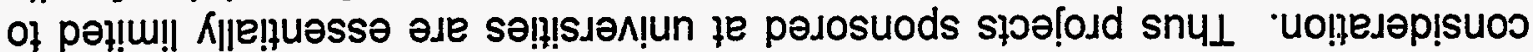

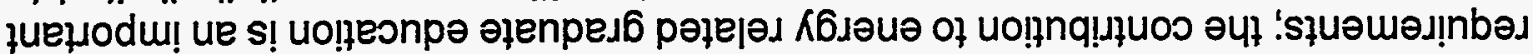

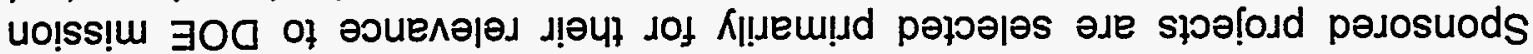

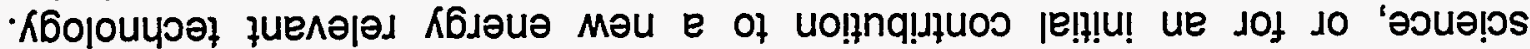

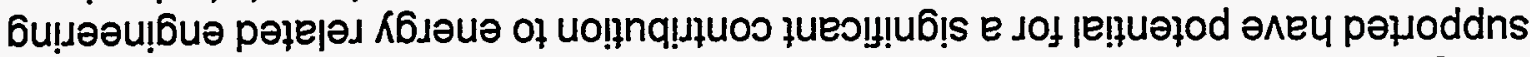

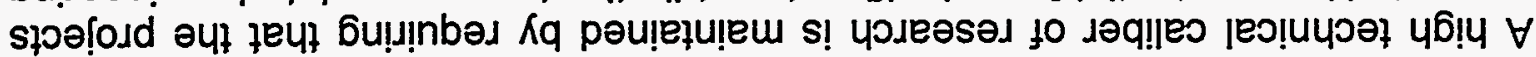

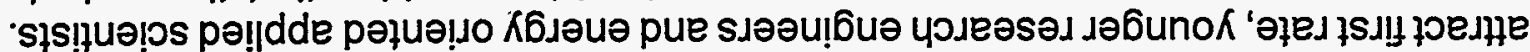

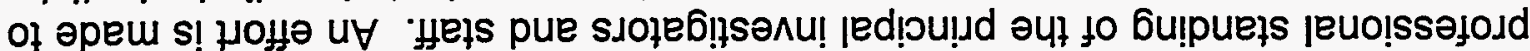

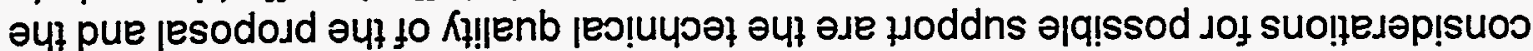

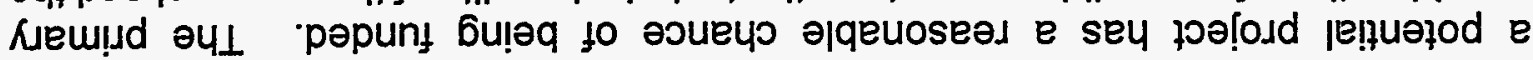

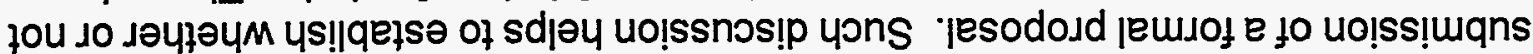



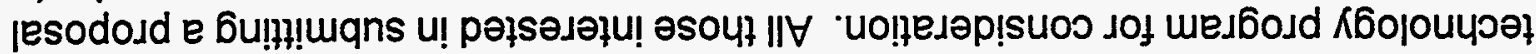

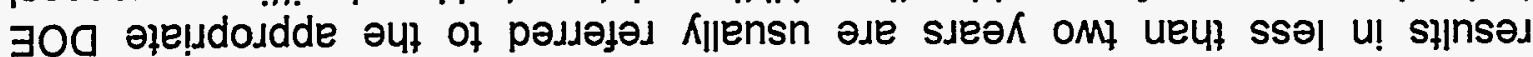

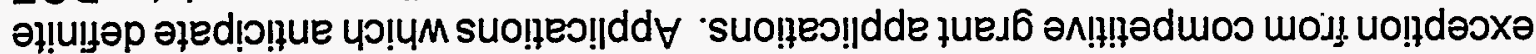

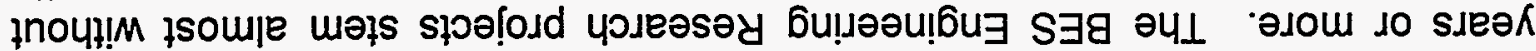
uə] se 6uol se fse| of pəłoədxə słoə⿳亠丷厂

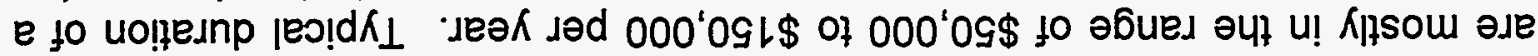

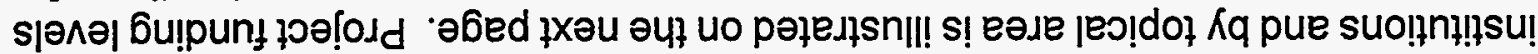

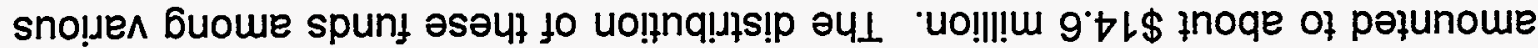

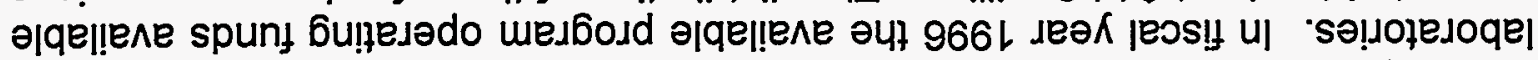

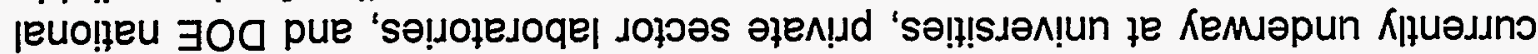

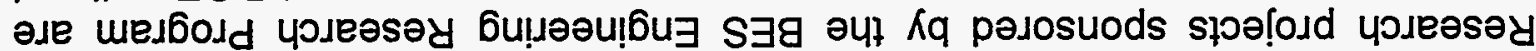

'pəjosuods uәəq әлеч !!

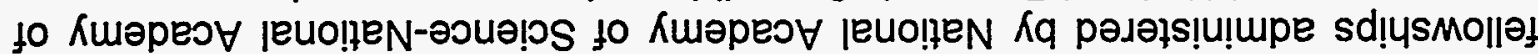

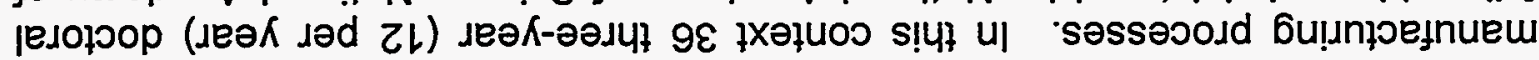

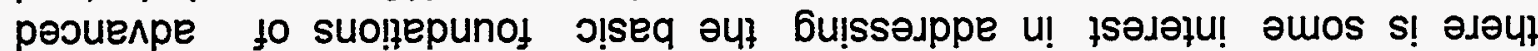

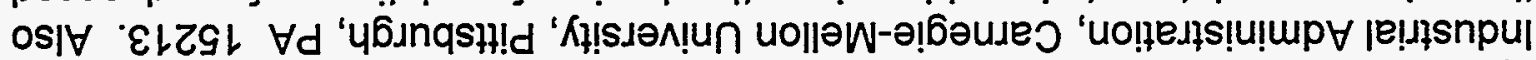

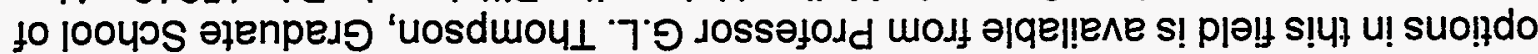

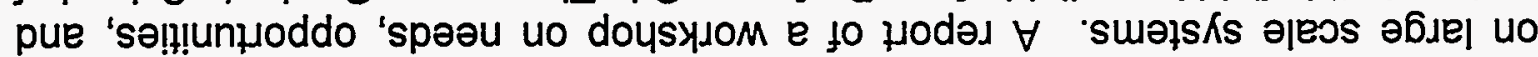

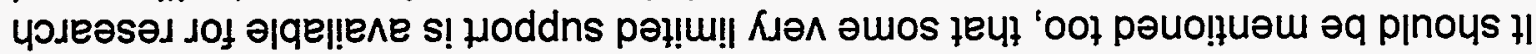

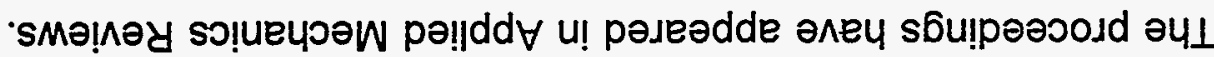

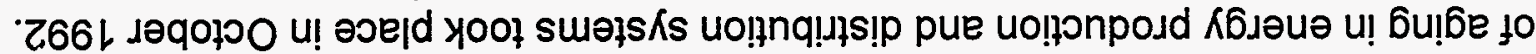

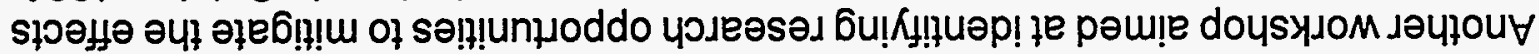




\section{ENGINEERING RESEARCH PROGRAM}

FY '96 BUDGET (\$000's)

BY INSTITUTIONAL TYPE

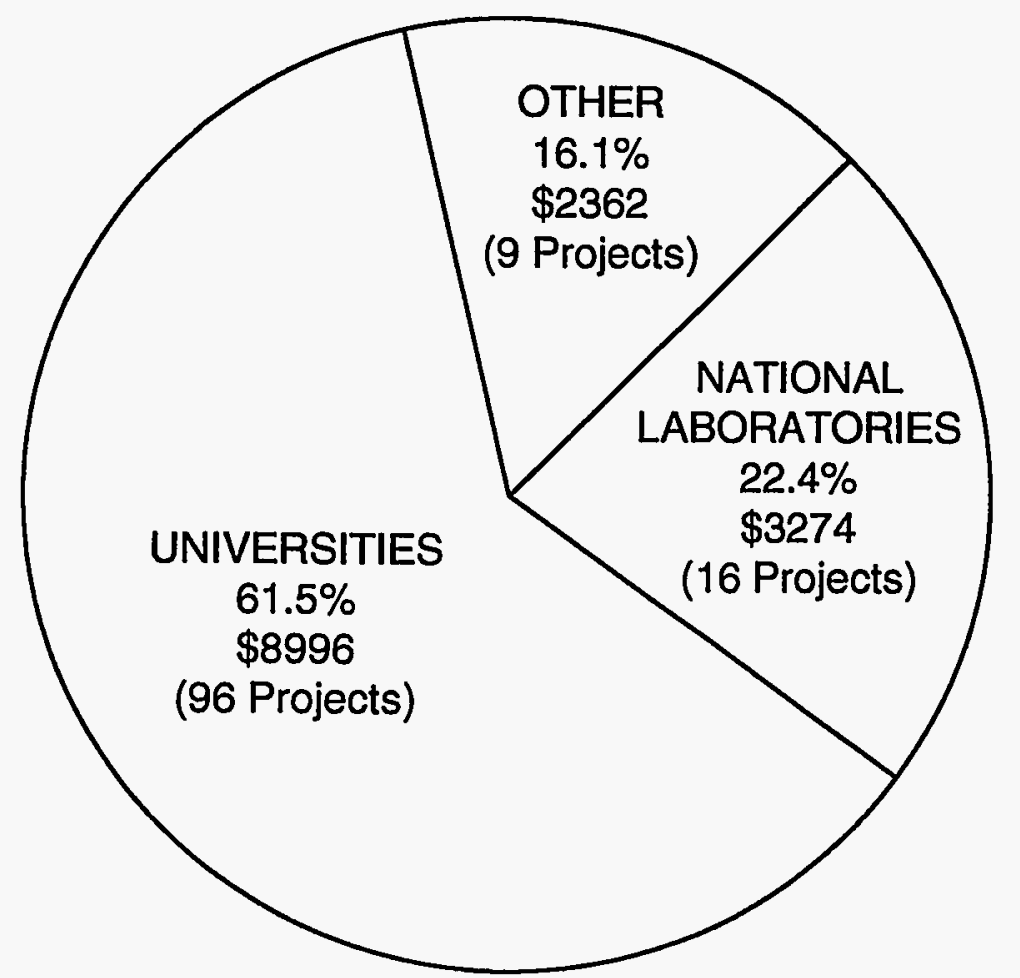

ENGINEERING RESEARCH PROGRAM FY'96 BUDGET BY TECHNICAL AREAS

$$
\text { (000's) }
$$$$
3561
$$$$
5082
$$$$
5988
$$

$\%$

24.3

34.7

41.0
NUMBER OF PROJECTS

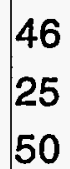




\section{Table of Contents}

University of Alabama . . . . . . . . . . . . . 1

University of Arizona . . . . . . . . . . . . . . . 1

Arizona State University . . . . . . . . . . . . . . 2

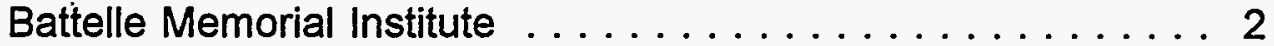

Brown University ..................... 3

University Of California, Berkeley ............ 3

University Of California, Los Angeles . . . . . . . . . 4

University Of California, San Diego . . . . . . . . . . . 6

University Of California, Santa Barbara . . . . . . . . . 8

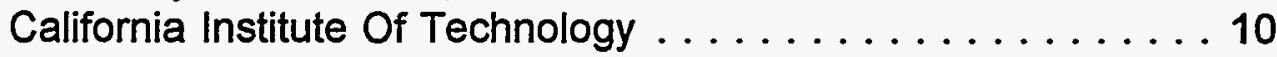

Carnegie Mellon University . . . . . . . . . . . . . 10

University Of Chicago .................... 11

Clarkson University . . . . . . . . . . . . . . 11

Cornell University $\ldots \ldots \ldots \ldots \ldots \ldots \ldots \ldots \ldots \ldots \ldots$

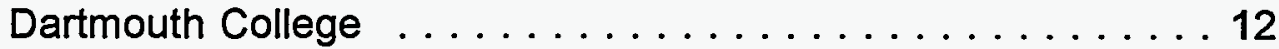

Duke University . . . . . . . . . . . . . . . . 13

Florida State University . . . . . . . . . . . . . 13

Georgia Institute of Technology . . . . . . . . . . 13

Idaho National Engineering Laboratory . . . . . . . . . . 14

University Of Illinois . . . . . . . . . . . . . . . . 17

Johns Hopkins University . . . . . . . . . . . . . 18

Robert H. Kraichnan, Inc. . . . . . . . . . . . . 19

Lawrence Berkeley Laboratory .............................. 19

Lawrence Livermore National Laboratory . . . . . . . . . 19

The Lovelace Institutes . . . . . . . . . . . . . . . 20

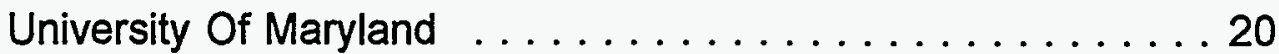

Massachusetts Institute Of Technology . . . . . . . . 22

University Of Minnesota . . . . . . . . . . . . . . . . 25

National Academy of Sciences/National Research Council . . . 26

National Aeronautics and Space Administration . . . . . . . 26

National Center for Manufacturing Sciences . . . . . . . . . . 27

National Institute Of Standards And Technology . . . . . . 27

The City University Of New York, The City College . . . . . 28

State University of New York . . . . . . . . . . . . 30

Northwestern University . . . . . . . . . . . . . . . 30

University Of Notre Dame . . . . . . . . . . . . . . . . 32

Oak Ridge National Laboratory $\ldots \ldots \ldots \ldots \ldots \ldots$ 


\section{Table of Contents-(continued)}

Pennsylvania State University $\ldots \ldots \ldots \ldots \ldots \ldots \ldots . \ldots 33$

Princeton University $\ldots \ldots \ldots \ldots \ldots \ldots \ldots \ldots \ldots \ldots$

Purdue University . . . . . . . . . . . . . . . . . . . . . 34

Rensselaer Polytechnic Institute . . . . . . . . . . . 35

Rice University . . . . . . . . . . . . . . . . 37

University Of Rochester . . . . . . . . . . . . . . . 37

Rockefeller University . . . . . . . . . . . . . . . . . . . 38

Sandia National Laboratories . . . . . . . . . . . . . . 38

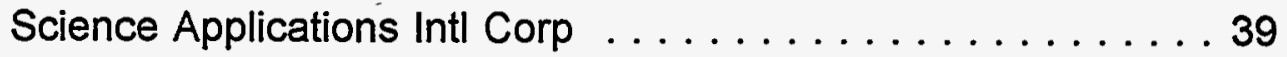

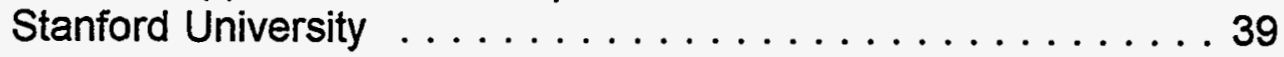

University Of Texas At Austin . . . . . . . . . . . . . . . . 42

University of Washington . . . . . . . . . . . . . . 43

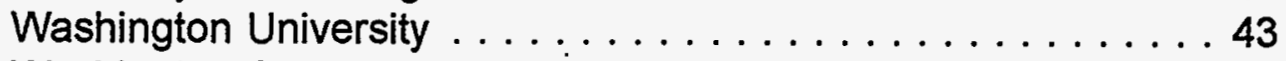

Washington State University $\ldots \ldots \ldots \ldots \ldots \ldots \ldots 44$

University Of Wisconsin . . . . . . . . . . . . . . 44 
University Of Alabama

Dept of Mathematics

Tuscaloosa, AL 35487

$\$ 118,461$

01-C

94-3

Hydrodynamic Instabilities and Coherent

Structures

\section{A. Frenkel}

The objective of this research is to further the fundamental understanding of stability properties of several far-from- equilibrium fluid systems which are relevant to energy engineering sciences. In particular, flows periodic in space and possibly in time are studied as models to gain insights into such turbulence phenomena as large-scale coherent structures, eddy viscosity, and the inverse cascade of energy. Film flows-such as core-annular ones-are important to, e.g., lubricated pipelining of viscous oils. The large-scale evolution equations for the different systems may exhibit common features, such as pattern formation and coherent structures.

Some of the results are as follows: A rigorous iterative method was suggested for the problems of periodic-flow stability. The possibility of negative isotropic large-eddy viscosity was demonstrated, resolving a rather long-outstanding question. A weakly nonlinear Landau-type theory was constructed for an intermediate-scale instability of a periodic flow. The spatial structure of the saturated disturbances of this non-uniform-flow is significantly different from previously known, uniform cases.

For film flows, a perturbative method capable of yielding both the evolution descriptions and the parametric conditions of their validity was suggested. A highly nonlinear evolution equation of a wavy flow down a cylinder was obtained. Its numerical simulations yielded an excellent agreement with experiments. They also revealed, for the first time ever in numerical simulations, irreversible coalescences of soliton-shaped coherent structures; these are now thought to play an important role in the wave dynamics of film flows. Also, a theory of a flow down an inclined plane was constructed. Simulations of the evolution equation showed a remarkable agreement with three-dimensional wave patterns observed in recent experiments.

Some fundamental questions concerning commonly used perturbative approaches were clarified. Certain deficiencies of well-known evolution equations were pointed out.

\section{University of Arizona}

Aerospace \& Mechanical Eng

Tucson, AZ 85721

01-B

Film Cooling in a Pulsating Stream

93-3

\section{Wygnanski, H. Fazel, A. Ortega}

In a joint effort - theoretical, computational and experimental - we are investigating mechanisms to suppress or enhance the heat transfer in a strong laminar/transitional wall jet flowing over an isothermal surface. Theoretically, we have explored the effects of temperature gradients on the mean flow and on the stability characteristics. Direct Numerical Simulations (DNS) were performed by adapting the Navier-Stokes codes to include the effects of heat transfer on wall jets that are actively controlled by periodic forcing. Experiments were carried out in a wind tunnel over an isothermal surface which can be either cooled or heated. The primary finding to date suggests that the Reynolds analogy does not apply to time mean quantities when the flow is unsteady; and that both the mean and fluctuating quantities of velocity. and temperature are coupled rather than independent as has been often assumed for theoretical solution of convective heat transfer problems. Selective forcing at the dominant instability modes showed that excitation of the inner (viscous) mode with $2 \%$ forcing level resulted in a reduction of the maximum skin friction by as much as $65 \%$ and an increase in the maximum wall heat flux of as much as $45 \%$. These investigations will be extended to strong turbulent wall jets. 
Arizona State University

Mechanical \& Aerospace Eng

Tempe, AZ 85287

$92-4$

Contiuum Damage Mechanics - Critical States D. Krajcinovic

Objective: Primary objective of the current research program is to examine a variety of critical states in mechanical response of brittle and quasibrittle solids containing a large number of cracklike micro-defects. More specifically, the focus of the ongoing research is placed on the determination of circumstances (type of loading, confinement level, shape and size of the specimen, thermal and environmental conditions, etc.) leading to the onset of critical states defined as a threshold connectivity at which a solid ceases to support external loads.

Technical Approach: Current applied mechanics / engineering practice in evaluating the mechanical failures of brittle and quasi-brittle solids emphasizes use of effective continuum theories coupled with the deterministic and highly idealized description of the defect geometry (such as doubly periodic arrays of penny-shaped cracks). In contrast, the approach selected in this research program accentuates the stochastic geometry of the microstructural disorder and its effect on the onset of macro-fracture and the type of the failure mode.

One of the important aspects of this research is to explore applicability of the novel methods of statistical physics (percolation theory, models of self-organized criticality, etc.) to micromechanical models. Some of the already obtained results provide connection between the mechanical parameters such as stiffness and damage variable and the percolation theory concepts such as the order parameter, excluded volume, etc.. This provides a set of rational criteria for the selection of the universal dimensional invariants needed to describe the onset of a certain class of failures. Secondly, use of the statistical methods (such as fractal and multifractal formalism) provide a superior and size-independent (intrinsic) description of the fluctuations in the stress field (stress concentrations) in the vicinity of the critical states. This aspect alone should provide a definitive answer related to the dependence of the order-disorder transition on the microstructuraltexture and/or boundary conditions.
In-summary, the selected approach provides the best hope of description of the universal aspects of the stochastic nature of the damage and its evolution in the vicinity of the critical state.

\section{Battelle Memorial Institute}

Engineering Mechanics Dept

Columbus, $\mathrm{OH}$ 43201-2693

$\$ 99,729$

01-A

94-3

An Investigation of History-Dependent

Damage In Time-Dependent Fracture

Mechanics

F. Brust, Jr.

In order to meet the demand imposed by future technology, new plants with increased energy efficiency must operate at relatively high temperatures. Additionally, the existing power generation equipment in the United States continues to age and is being used far beyond its intended life. Some recent failures have clearly demonstrated that the current methods for insuring safety and reliability of high temperature equipment is inadequate. Owing to these concerns, a thorough understanding of high temperature failure initiation and propagation in materials exposed to variable mechanical and thermal loading is very important.

In the past, the evolution of damage has been addressed through a macroscopic theoretical model (developed as part of this effort) which attempt to predict the crack growth and failure response of material components exposed to high temperature conditions. However, micromechanical processes such as diffusion of atomic flux into grain boundaries, elastic accommodation and creep deformation of the material and grain boundary sliding do contribute significantly to the nucleation and growth of voids leading to failure. Understanding gained by consideration of micro-mechanics of cavity growth is crucial for developing damage-based constitutive models as well as methodologies for life prediction of structural components. While the application of this understanding in estimating life of structural materials experiencing high temperature creep has met with some success, it is of limited use for structural components experiencing complex load histories under high temperature conditions. 
A micro-mechanical model accounting for rate-controlling microscopic processes has been developed as part of this effort. To date, both sustained and variable load histories have been investigated in two-dimensional geometries. The results illustrate the importance of accounting for nonlinear changes in geometry, grain-boundary diffusion processes, elastic accommodation of the surrounding material as well as more realistic constitutive laws for creep deformation. Current efforts involve investigating different load histories and three-dimensional effects. In addition, the ultimate goal of this effort is to establish a firm connection between the micro- and macro-mechanical models thereby leading to the development of appropriate methodology for life prediction of structural components exposed to high temperature conditions involving complex load histories.

\section{Brown University}

Dept of Chemical Engineering

Providence, RI 02912

$\$ 99,410$

01-A

95-3

Simulation and Analysis of Dyanmic Failure of Ductile Materials

\section{L.B. Freund}

A central goal in the mechanics of materials is the determination of parameters which characterize macroscopic failure of materials in terms quantifiable characteristics of their microstructure. The motivation is to establish which characteristics account for macroscopic failure, with a view toward improvement of failure resistance through material selection or microstructural design. In the present project, emphasis is on the behavior of ductile structural alloys under high rate loading conditions. Thus, the dominant mechanism of plastic deformation is crystallographic slip and material strength degrades through nucleation, growth and coalescence of micro-voids. Plastic strains in such processes can be large and strain localization is common. The approach is to adapt methodologies for analysis of elastic-viscoplastic systems to problems selected on the basis of their relevance to safety of pressure vessel and piping systems, to materials processing and metal forming technologies, and to structural reliability under dynamic loading. Initial emphasis has been on failure of an explosively loaded ring expanding under plane strain conditions, a configurationwhich has been studied experimentally. Calculations reveal that strain localization sites, or necks, are more pervasive under rapid loading, and the spacing of necks decreases with increase in loading rate. The influence of inertia on bifurcation of deformation states is also being investigated theoretically. The project is being carried out in collaboration with colleagues involved in experimental research on dynamic ductile failure at the California Institute of Technology.

\section{University Of California/B}

Dept of Chemical Engineering $\$ \$ 81,031$

Berkeley, CA 94720

06-A

$94-4$

Enzyme Adsorption and Activity at Liquid-

Liquid interfaces

H. Blanch

Our research examines the dynamics of protein and enzyme adsorption at an oil/water interface. Transient interfacial tension studies show that at short times (seconds), predicted diffusional effects are observed. At extended times (hours), adsorbed proteins form gel-like layers. Adsorption is accompanied by conformational changes and interactions between adjacent molecules, often resulting in the formation of a quasi-solid macroscopic skin at the interface. We use transmission electron microscopy (TEM) to analyze the skin layers collected from the oil/water interface. Microporous polymer-coated TEM grids serve as novel substrates to collect and observe these layers. The effects of skin formation on enzyme activity are currently being probed.

We also use fluorescence spectroscopy to follow protein adsorption and to gain information on protein molecular conformation in the adsorbed state. A novel total internal reflection fluorescence spectrometer (TIRFS) for liquid/liquid interfaces has been developed in our lab. This TIRFS design includes a stagnant water layer separated from a flow channel by a semi-permeable membrane. This apparatus enables the dynamics of protein adsorption to be followed while also giving us a means to test the reversibility of surface reactions in a non-invasive manner. In developing the TIRFS cell the mass transport has been tested using a simple fiuorescent marker (fiuorescein). The transport dynamics can be accurately described using a simple diffusion model. 
University Of California/B

Dept of Mechanical Engineering

Berkeley, CA 94720

$\$ 100,661$

01-B

95-3

Ultrashort Laser Heating and Phase

Change in Liquids

C.-L. Tien

The general objective of this research program is to achieve a better understanding of laser-liquid interactions from the thremal perspective. Two main tasks were proposed for investigation: the fundamental mechanisms of high-intensity, shortpulse laser heating of liquids and pulsed-laserinduced vapor-phase nucleation and growth. This report present the progress of the research for these two tasks.

Mechanisms of High-Intensity Laser Heating of Liquids

When the pulse duration of short-pulse, high power lasers approaches the characteristic molecular time scales of the material with which the laser interacts, traditional models of radiation absorption and energy transport must be reexamined. A complete understanding of heat generation and dissipation during this process is required for precise, efficient laser processing. The research of the past year has resulted in microscopically based models of radiation absorption and thermal transport in liquids. These models have been applied to create novel solutions to important engineering problems.

The model for liquids was developed to include consideration of the short time-scale phenomena of saturable absorption and multiphoton absorption in energy transport calculations. The multiphoton effect was proposed as a novel mechanism for imparting large amounts of energy into normally transparent liquids. Experiments were performed to confirm model predictions. A method for removing small amounts of liquid contaminants from metal or semiconductor substrates was computationally modeled. Finally, using predictions from the models of femtosecond $\left(10^{-15} \mathrm{~s}\right)$ laser interactions with semiconductors, a method of releasing failed microstructures through electronically-induced desorption of water was developed and demonstrated.
Pulsed-Laser-Induced Vapor-Phase Nucleation and Growth

Irradiation of a $\mathrm{KrF}$ excimer laser beam of nanosecond pulse duration on an absorbing solid surface immersed in a transparent liquid induces rapid thermal expansion and explosive vaporization of the liquid accompanied by pressure pulse generation. The pressure contribution due to the thermal expansion of the solid sample and bubble formation was measured by the probe beam deflection method as well as by a broadband piezoelectric transducer. The pressure generation is enhanced by the bubble expansion in the superheated water for laser fluences exceeding the bubble nucleation threshold. The bubble growth dynamics was examined by a novel, non-contact optical interference technique. This work quantified, for the first time, the transient behavior and growth rate of a submicron thick bubble layer in the nanosecond time scale. Experiments are now focused on the study of pulsed laser-induced cavitation and phase-change in absorbing liquids.

\section{University Of California/LA}

Mech, Aero \& Nuclear Eng Dept \$137,304

School of Eng \& Applied Science 01-C

Los Angeles, CA 90024-1597 96-3

\section{Basic Studies of Transport Processes in Porous Media \\ I. Catton}

The objective of this project is to develop an understanding of the goveming physical processes at a level appropriate for development of reliable macroscopic models for use in the analysis and design of engineered energy systems. The basis of the work is a hierarchical heterogeneous medium averaging methodology based on volume averaging theory (VAT) using second order turbulent models with Reynolds stresses and fluxes, and intermedia exchange in every pore space. Boundary and interphase conditions are incorporated at various scales leading to descriptions of momentum, heat and mass transport in porous media. Equations for laminar and turbulent filtration with two-temperature or twoconcentration diffusion in non-isotropic random porous media have been developed along with the statistical and numerical methodology needed to treat the fluctuation terms for various assigned 
random porous morphologies. The equations differ from those found in the literature. Current work considers both single- and two-phase flows and is both theoretical and experimental.

The models are being used to optimize some heat transfer devices by selecting the surface and media characteristics that yield high heat transfer and thermal capacity, and low pressure drop. Diesel engine regenerators and flow through or across bundles of roughened tubes are presently being modeled. An apparatus to measure the spacial distribution of flow momentum in a porous morphology has been designed and exercised. Data for silicon carbide foams, for use as a regenerator, are being obtained.

Results were obtained for transport of liquid (water) at a micro-scale pore diameter level. It was shown that the flow becomes non-Newtonian, effecting filtration at the smallest porous media scales. Analysis and comparison of modeling results for some different types of stochastic distributions of pore diameters show strong dependencies of velocity, mass transport coefficient and other media properties on the medium morphologies.

\section{University Of California/LA}

Mech, Aero \& Nuclear Eng Dept School of Eng \& Applied Science $\$ 0$

Los Angeles, CA 90024

06-C

93-3

Linear Kinetic Theory and Particle Transport in Stochastic Mixtures G. Pomraning

The goal in this research is to develop a comprehensive theory of linear transport/kinetic theory in a stochastic mixture of solids and immiscible fluids. Such a theory should predict the ensemble average and higher moments, such as the variance, of the particle or energy density described by the underlying transport/kinetic equation. The statistics to be studied correspond to $\mathrm{N}$-state discrete random variables for the interaction coefficients and sources, with $\mathrm{N}$ denoting the number of components in the mixture. The mixing statistics to be considered are Markovian as well as more general statistics.

In the absence of time dependence and scattering, the theory is well developed and described by the master (Liouville) equation for Markovian mixing, and by renewal equations for non-Markovian mixing. The intent of further work is to generalize these treatments to include both time dependence and scattering. A further goal of this research is to develop approximate, but simpler, models from the comprehensive theory. In particular, a specific goal is to formulate a renormalized transport/ kinetic theory of the usual nonstochastic form, but with effective interaction coefficients and sources to account for the stochastic nature of the problem. Numerical comparisons of all models will be made against Monte Carlo simulations which involve a straightforward average of solutions for a large number of physical realizations of the statistical mixing. Contact will also be made with experimental simulations of cloud-radiation interactions currently underway at another institution as part of DOE's global climate modeling initiative.

\section{University Of California/LA}

Physics Dept

Los Angeles, CA 90024

$\$ 75,000$

06-C

$96-4$

Nonlinear Waves in Continuous Media: Application to Stochasticity and Energy Concentration

\section{S. Putterman}

Nonlinear wave interactions in far off-equilibrium fluids are being studied with the goal of understanding the interplay between processes that concentrate energy (e.g. sonoluminescence), randomize energy (e.g. wave turbulence) and form localized states (e.g. solitons). The experimental discovery that strong sound waves generate picosecond fiashes of light is now being studied from the theoretical perspective with the goal of understanding how energy can focus by twelve orders of magnitude. Turbulence in nonlinear waves is being studied from both the theoretical and experimental directions. Goals include the development of a Fokker-Planck theory that includes intermittency as well as the observation of collective modes in turbulence that are analogous to second sound. High amplitude waves can also form self-localized states such as the breather and kink solitons, and domain walls which have recently been observed. Current efforts are aimed at extending these findings to systems which are two- and three-dimensional. This work proceeds from the experimental, analytical and simulational avenues of approach. 


\section{University of California/SD}

Scripps Inst of Oceanography

$\$ 185,500$

06-C

94-4

\section{Broadband Signals: Signal Processing in Chaos \\ H. Abarbanel}

The analysis of chaotic signals observed in measurements on physical systems is of importance in energy problems ranging from fluidized bed flows in fossil energy applications to determination of the natural climate variability to the uncovering of simple models for complex behavior in fiuid flows. This research has developed tools for this analysis which allows one to reconstruct the multivariate state space of a system from observations, time lagged, of a single dynamical variable. The time delay, the dimension, and properties of the strange attractor can all be determined from these data. The concept of unfolding the attractor using the method of global false nearest neighbors and then determining locally the number of dynamical degrees of freedom using the local version of this has brought to the study of complex behavior a robustness which allows it to be used in engineering analysis and design. The algorithms developed for this purpose have also been used in a variety of applications requiring the separation of a chaotic signal from another information bearing signal or 'noise'. The tool kit of these algorithms is being ported to a common interface for use in the energy related sciences.

\section{University Of California/SD}

Dept of App Mech \& Eng Sci

La Jolla, CA 92093

$\$ 137,639$

06-B

96-3

Fundamental Studies of Spray Combustion

\section{P. Libby, F. Williams}

This research involves a combined experimental and theoretical effort related to the behavior of fuel droplets in well defined but nonuniform flows. A test rig which permits a wide variety of investigations including nonpremixed, premixed and partially premixed systems in both laminar and turbulent streams represents the main experimental setup. A phase doppler particle analyzer which permits measurement of two velocity components, droplet diameter and number density is the principal instrumentation employed in our studies. It is supplemented by a gas chromatograph for measuring the concentrations of stable species and by thermocouples for temperature measurements. One recent research effort has been concerned with the reduction in NOx production in two stage flames by water droplets and has involved both experimental and numerical studies. A second effort has been deyoted to the oxidation of methanol in fuel rich premixed and nonpremixed flames. This research has exposed an important reaction path for the formation of $\mathrm{CH}$ and subsequently of NOx.

\section{University Of California/SD}

Dept of Chemistry, 0340

$\$ 102,612$

La Jolla, CA 92093

06-C

Noisy Nonlinear Systems

$96-4$

\section{K. Lindenberg}

The broad objective of this project is to investigate the interplay of nonlinear deterministic dynamics with spatial and temporal fluctuations.

Density fluctuations in binary reaction-diffusion processes in low dimensions lead to spatial and temporal anomalies. The effects of different initial conditions on temporal evolution have been analyzed. Away from equilibrium each initial condition (spatial distribution at one instant) places a distinctive imprimatur on the time evolution ofthe system.

The exploration of the manifestations of soft anharmonicity in nonlinear coupled arrays has continued with the investigation of the interplay of two coexisting energy-localizing (relative to a harmonic chain) mechanisms in such systems. One is the energy-driven mechanism that leads to the inhibition of dispersion and the enhancement of spatial coherence (e.g. via the formation of solitons). The other is the entropy-driven mechanism of stochastic localization that leads to spatially incoherent localization due to the preponderance of high amplitude vibrations in soft anharmonic systems relative to harmonic systems. The dynamics of the standard integrate-fire model and a simpler model (that reproduces important features of the integrate-fire model under certain conditions) of neural dynamics, have been analyzed in the presence of a deterministic time-periodic driving force and white background noise. Both models exhibit resonant phenomena in 
the first passage probability distribution and mean first passage time arising from the interplay of characteristic time-scales in the system.

\section{University Of California/SD}

Inst for Nonlinear Science, R-002

La Jolla, CA 92093

$\$ 96,091$

06-C

Structure and Modeling of Turbulence

96-3

\section{E. Novikov}

Well developed turbulent flows are encountered in many energy engineering enterprises, as well as in environmental, aerospace and naval engineering. The number of degrees of freedom for such flows is enormous and direct numerical simulations (DNS) are impossible now and in a foreseeable future.

One of the major goals of this project is to advance the knowledge of the structure of turbulence, aiming it at a subgrid-scale modeling for the large-eddy simulations (LES) and at a transformation from homogeneous to nonhomogeneous turbulent flows.

The developed approach for achievement of this goal includes: conditional averaging of the Navier-Stokes equations (NSE); use of Markov processes with dependent increments (consistent with NSE); breakdown coefficients (for a description of intermittency) and associated infinitely divisible probability distributions.

Among the obtained results are: probability distribution for the three-dimensional (3D) vectors of velocity increments, which has an unusual form and experimentally observed exponential asymptotics; a new scaling for experimentally and numerically observed 3D "vortex strings", 2D coherent vortices and coherent structures of scalar fields; the whole 3D conditionally averaged vorticity field (with fixed vorticity in one point) and its characteristics, obtained by DNS on the CM- 5 parallel computer, using finite differences on (256) ${ }^{3}$ grid; statistical characteristics of microcirculations; an imbedding of the phenomena of intermittency and scale similarity into the theory of infinitely divisible probability distributions; probability distributions and moments of the breakdown coeffcients for the energy dissipation, compared with experimental data for the Taylor-scale Reynolds number 3200 ; a hierarchy of subgrid- scale models, tested by DNS and by LES for isotropic and free-surface turbulence; an analytical transformation from homogeneous to nonhomogeneous turbulent flows, tested by data for free-surface turbulence in a presence of a solid boundary.

\section{University Of California/SD}

Inst for Nonlinear Science

La Jolla, CA 92093

$\$ 150,000$

$06-\mathrm{C}$

$96-3$

Spatiotemporal Wave Patterns: Models and

Analysis

M. Rabinovich, L. Tsimring

Key phenomena of pattern evolution and spatio-temporal disorder in nonequilibrium media and complex systems are investigated experimentally and theoretically. We consider the dynamics of defects against the background of regular two-dimensional patterns (rolls, hexagons, quasi-crystalline structures); the self-organization of an irregular ensemble of defects and the formation of domain walls, as well as the interaction of two-dimensional patterns with large-scale flows. Experiments are carried out with Faraday ripples, Benard-Marangoni convection, and an oscillating soap film. The experiments with soap film for example, demonstrate that for a given steady flexural mode pattern, increasing the amplitude of mode oscillation leads to the excitation of the planar vortices. For large enough amplitude in a square cell, one can see the spatio-temporal chaos.

Spatio-temporal patterns in lattice models of the cortex are also addressed. Of primary interest here are the phenomena associated with the birth of regular, ordered behavior in an ensemble consisting of elements with chaotic individual dynamics (neurons). The distinguishing feature of such "media"' is the coupling between the elements that is not traditional for nonlinear dynamics. Namely, the coupling through chemical synapse (in contrast to ordinary diffusive coupling) is nonlinear and, generally, inertial. 
University Of California/SD

Dept of Physics

La Jolla, CA 92093

$\$ 119,338$

$06-\mathrm{C}$

$96-3$

Experimental Study of 2D Traveling-Wave

Patterns in Binary Fluid Convection

C. Surko

This research involves study of convection in fluid mixtures of ethanol and water in which the fluid motion takes the form of traveling-waves. This is a model system for investigation of nonequilibrium traveling-wave phenomena, and it provides important insights into the behavior of double diffusive systems in which the transport occurs on two different time scales. As a fluid dynamical system, it is closely related to atmospheric and oceanographic flows; and as a model for traveling wave dynamics, it provides insights useful in understanding other traveling-wave systems, such as large-aspect-ratio lasers.

Recent work centers on study of 2-D traveling wave patterns in a large aspect ratio container. When convection is initiated, the pattern is highly disordered, consisting of small domains and complicated superpositions of traveling-waves. As the pattern evolves, the domains increase in size, until the initially turbulent state gives way to a highly ordered multi-domain pattern. Numerical techniques have been developed to calculate the complex order parameter of the patterns and to identify and track phase defects. This is an effective technique for quantifying the level of disorder, and it may be applicable to a wide variety of traveling-wave systems. The dynamics of the domain boundaries are also being investigated using a variety of advanced numerical techniques.

\section{University Of California/SD}

Institute of Nonlinear Science

$\$ 101,900$

La Jolla, CA 92093-0402

03-A

95-3

\section{Modeling of Process Control}

L. Tsimring

Traditionally, for ease of operation and analysis, technological systems (manufacturing systems, communication systems, etc.) have been designed to behave in as linear a manner as possible. Increasingly tight specifications, environmental considerations and economic pressures are pushing the operational windows into regions where assumptions of linearity tend to break down. Despite a flurry of activity in the area of nonlinear dynamical systems (both analysis and control system design) we see very few applications of these new results. One important reason is the lack of suitable nonlinear dynamic models. The objective of this project is the development of tools for building nonlinear models based on input/output data from systems which may exhibit chaotic or nonchaotic dynamics, to implement the algorithms in efficient software packages and to apply them to a wide range of examples, primarily from communications and process control. These examples are not just academic test cases which provide feedback on the direction of the theoreticaldevelopments, but constitute by themselves important technological problems. The varied background of the investigators, the interdisciplinary approach, and the emphasis on tools, soffware and applications hold high promise for a broad impact of the proposed research.

One way to control a system is to synchronize it with a known system or a dynamical model. We develop new tools for analyzing synchronized behavior of nonlinear chaotic systems. These tools can be applied to characterize chaotic synchronization in a wide class of dynamical systems and also to controlling nonlinear systems. We test these tools in experiments with electronic circuits.

We proposed a new data-based algorithm for trajectory planning of a nonlinear system. This algorithm allows one to build an input-output model directly from data and drive the system towards the desired trajectory using an accessible control parameter.

\section{University Of California/SB}

\author{
Dept of Physics \\ Santa Barbara, CA 93106 \\ Bifurcations and Patterns in Nonlinear \\ Dissipative Systems \\ G. Ahlers, D. Cannell
}

$\$ 143,300$

06-C

93-5

This project consists of experimental investigations of heat transport, pattern formation, and bifurcation phenomena in non-linear non-equilibrium fluid-mechanical systems. These issues are studies in Rayleigh-Benard convection, using both 
pure and multicomponent fluids. They are of fundamental scientific interest, but also play an important role in engineering, materials science, ecology, meteorology, geophysics, and astrophysics. For instance, various forms of convection are important in such diverse phenomena as crystal growth from a melt with or without impurities, energy production in solar ponds, flow in the earth's mantle, geo-thermal stratifications, and various oceanographic and atmospheric phenomena. Our work utilizes computer-enhanced shadowgraph imaging of flow patterns and high-resolution heat transport measurements.

We studied convection in a gas $\left(\mathrm{CO}_{2}\right)$ under pressure (about 30 bar) with a Prandtl number $\mathrm{Pr}=1$, in a very large aspect ratio sample (radius/height up to 150). For parameter values where time-independent parallel straight rolls were theoretically predicted to be stable, we found a state of spiral-defect chaos (SDC). These experimental results have stimulated a great deal of theoretical work, using both analytical and numerical methods and various model equations for convection.

We extended our experiments on SDC to small values of Pr by using mixtures of gases. If one component of the mixture has a large and the other a small molecular mass, values of $\mathrm{Pr}$ as small as 0.14 can be reached. The first appearance of SDC shifts closer to the onset of convection as $\mathrm{Pr}$ decreases. This gives information about the origin of SDC, but has not yet been explained quantitatively.

We investigated convection in a nematic liquid crystal in a horizontal magnetic field $(H)$. We found excelent agreement with recent theoretical predictions for the bifurcation line $R-C(H)$, and for the convection-roll orientations as a function of $\mathrm{H}$. We also used this system to study convection when two phases (the nematic and isotropic phases) are present in the cell. Convection in the presence of a first-order phase change is relevant to convection in the earth's mantle; ours are the first quantitative experiments relevant to this important problem.

\section{University Of California/SB}

Dept of Chemical \& Nuc Eng

$\$ 109,500$

Santa Barbara, CA 93106

$01-\mathrm{C}$

95-3

Wave Turbulence Interactions

S. Banerjee

The work is part of an ongoing research effort aimed at understanding transport processes across a gas-liquid interface. The research includes both experimental and computational studies focusing on the effects of waves on turbulence in a liquid stream. Two- and three-dimensional mechanically generated waves, spanning the range of conditions from sinusoidal waves to micro-breaking are studied. Experimental work involves using a 3-D LDA synchronized with ultra-sonic surface position measurements. Flow visualization with micro-bubbles and high speed video are used for direct examination of flow structures. DPIV technique are further applied to the flow visualization video images for in-depth quantitative flow field analysis.

Experimental results indicate that the turbulence intensity away from the wall was substantially increased as a result of the waves, especially close to the interface, which suggests possible enhancement of interface scalar transport. The increase appears to be a consequence of turbulence production in the outer flow region due to wave-induced Reynolds stress. Turbulence intensity in the wall region and turbulence wall structures were not significantly affected. Wave-induced turbulence was also seen in a laminar flow by flow visualization

Direct numerical simulation of gas and liquid flowing across a mobile interface is being conducted. The results will allow more detailed examination of the effects of waves on turbulence production. 


\section{California Institute of Technology}

Graduate Aeronautical Laboratories

Pasadena, CA 91125

$\$ 98,381$

01- A

Dynamic Failure Characterization

of Ductile Steels

\section{A. Rosakis}

The goal of the current research program is to study dynamic crack initiation in ductile steels (304 Stainless Steel and A 533 B Steel) at different loading rates and to establish appropriate dynamic fracture criteria. A variety of infrared and visible optic methods, and high speed photography are used in this study. As part of our work we are developing novel experimental methods to achieve our tasks.

The precracked steels specimens are subjected to dynamic three point bend loading by impacting them in a drop weight tower. This impact results in deformation followed by fracture initiation. During the dynamic deformation and failure process the time history of the transient temperature in the vicinity of the crack tip is recorded experimentally using high speed infrared detectors. Meanwhile, measurements of the deformation field are made simultaneously using the optical technique of Coherent Gradient Sensing (CGS). The dynamic temperature trace is used to determine the time history of the dynamic J-integral, $\mathrm{J}^{\mathrm{d}}(\mathrm{t})$, and to establish the time of fracture initiation and the dynamic fracture initiation toughness, $J^{d}(t)$ initiation. Currently we are validating these results through comparison with the simultaneously performed CGS measurements. Our ultimate goal is to establish the strain rate dependence of the dynamic fracture toughness.

Further confirmation of the experimental observations will be provided by dynamic, three-dimensional computational modeling of our specimen configuration and the loading history. Much of this numerical computation will be done in collaboration with the research group of Prof. $L$. B. Freund at Brown University.

\section{Carnegie Mellon University}

Chemical Eng Dept

Pittsburgh, PA 15213

$\$ 165,000$

03-A

94-4

Systematic Process Synthesis and Design

Methods for Cost Effective Waste

Minimization

\section{Biegler, I. Grossmann, A. Westerberg}

This project is developing a novel integrated approach for process synthesis and design to address recent environmental challenges. The approach provides rigorous trade-offs among raw material and energy costs, capital investment and waste treatment. Issues of waste minimization addressed include:

1. Economic design of reactor networks that minimize conversion of raw materials to waste byproducts.

2. Synthesis of separation sequences to isolate, redirect and recycle byproducts.

3. Integrated synthesis and design of reaction, separation and energy systems with environmental and operability concerns, also dealing with process uncertainty.

Superior reactor designs can have the greatest impact for process improvement, both from environmental and an economic perspective. A quantitative targeting approach for reactor networks is permitting the reduction of waste byproducts at the source. The second task concentrates on the synthesis of flexible separation processes (displaying azeotropic and liquid/liquid behavior) - with efforts to overcome the combinatorial explosion of alternatives, each of which requires a very large and difficult optimization be solved. The third task combines structural optimization and problem decomposition at various modeling levels in order to screen alternatives based on economic, environmental and operabilty trade-offs. This approach, which is being applied to combined cycle utility plants, includes the development of computational strategies for optimizing flexible designs that must cope with changes in demands and waste treatment requirements. 


\section{University Of Chicago}

The Enrico Fermi Institute

Chicago, IL 60637

$\$ 169,744$

$06-\mathrm{C}$

96-3

Fundamentals and Techniques of Nonimaging Optics

R. Winston

Nonimaging optics departs from the methods of traditional optical design to develop techniques for maximizing the collecting power of concentrating elements and systems. Designs which exceed the concentration attainable with focusing techniques by factors of four or more and approach the theoretical limit are possible. Our theoretical work on nonimaging designs has led to our group experimentally demonstrating ultra-high flux from sunlight which exceeds previous results by substantial factors. Our "tailored edge ray" method of nonimaging design has advantages for many applications, including solar pumped lasers. Our work on generalized radiance and instrument functions has contributed to a better understanding of the foundations of radiometry.

\section{Clarkson University}

Dept of Chemical Engineering

Potsdam, NY 13676

$\$ 63,125$

01-C

94-3

Gas and Solids Holdup in Three Phase

Bioreactors

J. McLaughlin

The goal of this research is develop mathematical modeling and tools that can lead to a better understanding of three phase Bioreactors. Fluidized beds that use gel beads containing bacteria are of particular interest. The bead Reynolds numbers are order unity. Thus, models developed for particle Reynolds numbers that are large compared to unity do not correctly predict the hydrodynamics of the Bioreactors.

A goal of the project is to understand the interaction between gas bubbles and gel beads. $A$ fluidized bed was constructed and used to measure the rise velocity of air bubbles. Translucent (bacteria free) gel beads made it possible to study bubble trajectories at solids volume fractions up to $50 \%$. The Thomas effective viscosity model was found to be useful for correlating the bubble rise velocity over the size range of interest. The success of the effective medium model motivates the study of bubble motion in clear liquids.

A computer simulation program is being used to simulate axisymmetric flow around an isolated bubble. It incorporates the effects of surfactants on the bubble motion. The simulations agree with published studies of bubble motion in both pure and impure water. The program has been used to compute wake volumes for bubbles in tap water and solutions of Triton $X-100$ in distilled water. The amount of surfactant needed to produce flow separation was determined for a specific bubble size.

\section{Cornell University}

Mechanical \& Aerospace Eng

$\$ 86,232$

Ithaca, NY 14853

$01-\mathrm{A}$

94-3

Nonlinear Dynamics of Fluid-Structure

Systems

F. Moon

Two principal experiments were carried out under this grant during the second year of this project:

i) Forced vibration of a single flexible tube with internal flow and ii) Cross flow measurements of chaotic vibrations of a flexible tube in a five tube row.

i) In the first set of experiments, begun in the first year, we explored multifractal phenomena, which describe the transition from quasiperiodic to chaotic vibrations. Quasiperiodic vibrations were observed in earlier experiments by Dr. G. Scott Copeland in flow through a long tube with an end mass. These results were reported an a Ph.D. dissertation in Summer 1990. By introducing a periodic-forcing of the tube in an adaptation of this experiment, we were able to more easily study this transition from quasiperiodic to chaotic vibration. This study was motivated by work in the mid 1980's on forced Rayleigh-Bernard flow which showed a linkage between quasiperiodic motion and the circle map.

In the forced tube experiment we were able to show a similar connection to the circle map, and in particular, the multifractal nature of the breakup of the torus in c phase space. A multifractal is a distribution function which is described by a set of points with a continuous set of fractal dimensions. 
Our observations of multifractal behavior were, we believe, the first to be observed in fluid-structure vibrations.

In January 1992, Mr. George Muntean visited Argonne National Laboratory and reported to Dr. S.S. Chen our findings on muiltifractal measurements. He subsequently presented a paper at the DOE Grantees meeting this past spring. A revised paper has been submitted to the Journal of Fluids and Structures, and is currently under review.

ii) The design of the cross-flow, tube row experiment began last summer and was completed this fall. These experiments parallel the work of Cai and Chen (1992). In the Cornell experiments the center tube of a five-tube set suspended on a flexible rod. The tube motion is limited by motion stops, thus introducing a strong nonlinearity in the tube stiffness. Preliminary experiments were carried out in water. The vibration amplitude versus flow velocity shows the instability onset (Hopf bifurcation) and the vibration saturation when the impact constraints limit the chaotic motion. This response was found to depend on whether the flow velocity was increasing or decreasing.

The vibration frequency was found to depend on the flow velocity. There is some evidence that the periodic motion at the onset of fiutter to chaotic motion transition occurs through quasiperiodic vibration.

At the present time we are carrying out the calculations of the fractal nature of the Chaotic motion with the goal of establishing the low dimensional nature of the dynamics. At the same time we will correlate our findings with a similar experiment at Argonne National Laboratory to establish the validity of the fractal dimension technique in determining chaos in such flows. The next stage of this work will involve experiments in cross flow of air past a row of tubes as described in the next section on our proposed research for the third year of this project.

In 1993 a new wind tunnel facility has been constructed and we are investigating nonlinear dynamics of a tube row in cross flow.

\section{Cornell University}

Sibley School of Mech \& Aero Eng $\$ \$ 112,000$

Ithaca, NY $14853 \quad$ 01-C

94-3

Experimental Studies of Reynolds Number

Dependence of Turbulent Mixing and

Transport

Z. Warhaft

Our experimental studies of the structure of turbulence and its mixing properties are motivated by a desire to better understand the fluid mechanics of chemical reactions, combustion and environmental pollution.

In the past year we have generated high Reynolds number grid turbulence by means of an active grid, first described by Makita. Triangular wings are attached to the grid bars which rotate and flap in a random way. Turbulence Reynolds numbers of 40,000 , (corresponding to a Taylor microscale Reynolds number of 800 ) have been realized, and the evolution of turbulence up to this value (which is comparable to Reynolds numbers observed in the atmosphere and large industrial flows) has been studied. In particular we have traced the evolution of the internal intermittency, which plays a major role in the dissipation of high Reynolds number turbulence. We have also studied passive scalar mixing down a mean gradient and we are presently studying heat dispersion from a line source.

\section{Dartmouth College}

Thayer School of Engineering $\$ \$ 125,180$

Hanover, NH 03755

01-C

$95-3$

Two-Phase Potential Flow

\section{G. Wallis}

The objective is to develop a mature science of two-phase potential flow, based on fundamental theory and tested by experiment.

A theory has been developed for the "drift force" on any object, in an inviscid flow with weak vorticity, in terms of the added mass tensor for the object. An earlier error in calibration having been corrected, measurements of the force on a set of streamlined objects in a shear flow agree with the theory. 
Exact solutions have been obtained for flow past a disc at an angle to a flow. They serve to illustrate and confirm the nature of the three-dimensional drift which displaces vortex lines and causes the force. CFD has been used to simulate the experiments. The measured forces are confirmed, but with some dependence on nodalization which needs further work.

The results display the vorticity patterns predicted by theory. Comprehensive CFD investigations are planned to check the predictions for a broad set of conditions, explore the limits of validity, and suggest further key experiments.

\section{Duke University}

Dept of Physics

Durham, NC 27706

Experimental and Analytical Investigations of Flows in Porous Media

\section{R. Behringer, G. Johnson, J. Georgiadis}

Research Objectives: The objectives of this project include the characterization, both theoretical and experimental of fluid flows through porous media. The particular focus is on fluid convective flow in porous media. Convection in porous media occurs, for instance, when a layer of fluid-saturated porous media is heated from below.

The characterization of porous convections includes both pure fluids and mixtures of different fluids. Long term benefits will be an improvement in the theortical and practical applications of flow in porous media in the presence of temperature gradients. Relevant practical situations include petroleum and hydrology problems where heating occurs.

Scientific Approach: The primary approach is experimental, and involves the use of Magnetic Resonance Imaging (MRI) as a powerful new tool to see the flow inside of porous media. The project involves the use of a MRI apparatus and a precision thermal apparatus for carrying out the convection experiments. Additional theoretical work now includes an analysis of the convective model equations with the goal of understanding recent experimental results. These results show a strong coupling between the solid structure of porous media and the flow states.
Current Status of Project: Recent work has been described in Shattuck et al. Phys. Rev. 75, 1934 (1995). These experiments show the strong coupling between pore space structure and flow patterns. An additional longer paper is in progress. This work forms the basis of Mark Shattuck's thesis. Continuing work will focus on experimental MRI studies of binary mixture convection in porous media.

\section{Florida State University}

$\begin{array}{lr}\text { Supercomputer Computations } & \$ 100,000 \\ \text { Research Institute } & 06-C \\ \text { Tallahassee, FL 32306 } & 95-5\end{array}$

Theoretical and Computational Studies of Pattern Formation

J. Viñals

We seek to improve our understanding of the mechanisms underlying the formation of complex spatio-temporal patterns in systems that are driven outside of thermodynamic equilibrium, to characterize the macroscopic properties of such states, and to apply the methodology to situations of interest in Materials Science, materials processing and Engineering. Two avenues of research are being pursued: Patterns and chaos in fluids, and an order parameter formulation of two-phase fluids. In the former, we utilize amplitude and order parameter equations to describe the formation of patterns, their instabilities and the transition to chaotic states in extended systems. In the latter, we introduce and order parameter formulation to describe moving boundary problems in two phase fluids, and consider microstructure evolution in systems not readily tractable with conventional hydrodynamics.

\section{Georgia Institute of Technology Computational Mechanics Center $\$ \$ 85,000$ Atlanta, GA 30332 \\ 94-3}

An Analytical-Numerical Alternating Method for 3-D Inelastic Fracture and Integrity Analysis of Pressure-Vessels and Piping at Elevated Temperatures

\section{S. Atluri}

Current and future power generation plants require efficient operation so that energy savings may be realized. In addition, power generation equipment 
in the US continues to age, creating operational dangers for the working staff as well as greater potential for power outages. Current methods to ensure safe operation of these plant components which operate in the nonlinear material regime are simplistic, and hence, not very reliable. This program is developing advanced analytical tools which can be used to reliably assure safety of future plants as well as aging plants. The finite element alternating method is the state-of-the-art methodology for determining stress intensity factors for two and three dimensional crack growth problems. This method has permitted accurate and simple analyses of linear fracture problems to be made so that sophisticated reliability assessments of operating equipment may be made. This program has extended the finite element alternating method so that it may now be used in the nonlinear regime, i.e., the non-linear finite element alternating method. With this new methodology, sophisticated damage and fracture assessments can be made for components which experience failures in the elastic-plastic and high temperature .creep regime. This is truly a revolutionary advance to the fracture assessment field.

Currently, sophisticated fracture assessments are being made using advanced fracture theories such as the $T^{*}$-integral which were previously unattainable. The methods are being verified by comparison of predictions to experimental results. It is anticipated that these advances will permit the designer to make sophisticated fracture assessments in the future with a minimum of effort.

\section{Idaho National Engineering Lab} $\begin{array}{lr}\text { Energy Res \& Applications Dept } & \$ 265,000 \\ \text { Idaho Falls, ID } 83415 & 06-A\end{array}$ Idaho Falls, ID $83415 \quad 93-3$

\section{Modeling of Thermal Plasma Processes}

\section{Chang}

Optimization of thermal plasma processing techniques requires a better understanding of the space- and time-resolved fluid flow and temperature distributions in the plasma, and of interactions between the plasma and injected or condensed particles. The present research is directed toward the development of a comprehensive theoretical and computational model capable of providing such information. The plasma is represented as a two-temperature multicomponent ideal gas governed by the Navier-Stokes equations. In contrast to much of the earlier work, local thermodynamic equilibrium is not assumed. Multicomponent diffusion is represented by a self-consistent effective binary diffusion approximation, suitably generalized to include ambipolar diffusion of charged species. A generalized formulation for diffusion in external electromagnetic fields has also been developed. Dissociation, ionization, and other chemical reactions are fully coupled to the plasma flow and are treated by a new general implicit chemistry algorithm. Extensions of this algorithm to two-temperature plasmas and heterogeneous reactions (such as particle condensation) are in progress. Discrete particles interacting with the plasma are represented by a stochastic particle model which allows for statistical distributions of particle properties. Applications to date include simulations of plasma spraying in both conventional and modern high-speed torches, as well as studies of chemical, thermal, and excitation nonequilibrium in thermal plasmas. Future applications to plasma spraying of functionally gradient materials, reactive spraying, and plasma synthesis are also planned.

\section{Idaho National Engineering Lab}

$\begin{array}{lr}\text { Applied Physics \& Optics Unit } & \$ 478,000 \\ \text { Idaho Falls, ID 83415 } & 06-A \\ & 93-3\end{array}$

Fundamentals of Thermal Plasma Processing

J. Fincke

This project is the experimental portion of a coordinated experimental-theoretical research project on thermal plasma processing of materials. This work is primarily focused on the development of advanced diagnostic and computational techniques and their application to obtain a better and more detailed understanding of the fundamental physical and chemical processes occurring in nonequilibrium thermal plasmas with entrained particles. The techniques thus developed and the information and insights they provide, can then be directly applied to process design, optimization, and scale-up. The diagnostic and computational techniques already developed under this project now represent the state of the art in this area. 
During the next five years of this project, we propose to further extend and generalize these techniques to permit their application to several additional topics of timely importance in the thermal plasma processing of materials, namely (1) functionally gradient materials (FGMs), (2) reactive plasma spraying, and (3) plasma chemical synthesis of nanophase materials. These topics share some common features and physics which make it efficient and cost-effective to consider them together. They forma a natural progression and will be pursued sequentially in the above order, but with significant overiap.

\section{Idaho National Engineering Lab}

$\begin{array}{lr}\text { Materials Science Division } & \$ 249,000 \\ \text { Idaho Falls, ID } 83415 & 03-A \\ & 93-3\end{array}$

Application of Intelligent Control Systems to Mixed-Culture Bioprocesses

J. Johnson, D. Stoner

Even though the control of biologically-based processes is an extremely complex, multi-task activty, conventional control systems operate at the most rudimentary of levels. That is, standard set point control and alarm systems are used to monitor and maintain process parameters, while process performance and recovery from upset conditions are dependent on the operator's judgment and experience. Artificial intelligence has the potential of incorporating a human's experience and rationale for the supervision of a bioprocess into a computer controlled system, thus, radically changing the manner in which bioprocesses are supervised and controlled. The challenges that confront the development of intelligent systems for bioprocessing control are due to the limitations of the intelligent technologies; the manner in which these technologies may be integrated into a control system; and the limitations inherent in the mathematical models that are used to describe microbial metabolism and activity.

The overall goal of this program is to demonstrate that the productivity of bioprocesses can be improved by integrating intelligent systems with conventional bioprocess control technologies. In addition, the project will demonstrate the application of intelligent control to a complex system that no analytic model can adequately describe. Investigators at the Idaho National
Engineering Laboratory have designed, built and evaluated hierarchical hardware and software systems for the intelligent control of continuous flow, stirred tank reactors. The control system was developed and evaluated using Thiobacillus ferrooxidans, a microorganism for which there is a moderate amount information. This learningbased control system was successfully applied to an uncharacterized culture of iron-oxidizing thermophilic microorganisms.

The hierarchical control systems had, as their lower level platform, an integrated system of set point control, data acquisition, data analysis and equipment diagnostics programs. The control system used on-line sensors and off-line measurements to determine the state of the system. A two-level control system determined what actions were required to meet process goals. These actions were carried out by computer controlled actuators, including pumps, gas-flow valves, a heater, and a stirrer. The upper level of the control system, the supervisory control system, used stochastic learning to determine what the system parameters, e.g., $\mathrm{pH}$, dilution rate, and temperature, should be, based on the state of the system. Lower level fuzzy systems and standard classical methods implemented the controllers that operated the system. A diagnostic system analyzed the sensor data for inconsistencies and provided a log of the system operation.

\section{Idaho National Engineering Lab}

Materials Technology Group $\$ \$ 132,000$

Idaho Falls, ID 83415-2218 01-A

93-3

Elastic-Plastic Fracture Analysis Emphasis

\section{on Surface Flaws}

W. Reuter, J. Epstein, W. Lloyd

The objective is to improve design and analytical techniques for predicting the integrity of flawed structural components. The research is primarily experimental, with analytical evaluations guiding the direction of experimental testing. Tests are being conducted on materials ranging from linear elastic to fully plastic. The latter extends beyond the range of a J-controlled field. Specimens containing surface cracks are used to simulate the fracture process (crack growth initiation, subcritical growth, and catastrophic failure) that may occur in structural components. 
Metallography and microtopography techniques have been developed to measure crack tip opening displacement and crack tip opening angle for comparison with analytical models. Moiré interferometry techniques are used to evaluate and quantify the deformation in the crack region. These studies have resulted in the ability to predict crack growth initiation of specimens containing surface cracks using constraint and fracture toughness data obtained from standard fracture toughness specimens. Results are being transferred to industry in the form of an ASTM Test Standard on Surface Cracked Specimens (Structures) that is presently being developed. Future research will focus on predicting the stable crack growth process in base metal and in weldments.

Due to the complexity of studying the fracture process in weldments, diffusion bonded specimens were used initially to simulate a weldment. This provided an opportunity to study the fracture process in a model weldment (two dissimilar materials, e.g., base metal and weld metal) of either a butt weld or a single " $V$ " groove geometry that contained neither a heat affected zone nor residual stresses. This work has been completed and now the focus is on actual weldments of $A 710$ steel. Two weldments have been fabricated with one having matched weld metal and the second an overmatched weld metal. Characterization of the microstructure and of local tensile properties is presently in progress. Testing of fracture toughness specimens, specimens containing surface cracks, and modified specimen geometries is planned for the future.

\section{Idaho National Engineering Lab Materials Technology Group Idaho Falls, ID 83415 $\$ 517,000$ 03-A \\ Intelligent Control of Thermal Processes 93-3}

\section{H. B. Smartt, J. A. Johnson}

This project addresses intelligent control of thermal processes as applied to gas metal arc welding. Intelligent control is defined as the combined application of process modeling, sensing, artificial intelligence, and control theory to process control. The intent of intelligent control is to produce a good product without relying on post-process inspection and statistical quality control procedures, by integrating knowledge of process engineering practice and process physics into sensing and control algorithms. The gas metal arc welding process is used as a model system; considerable fundamental information on the process has been developed at INEL and MIT during the past ten years. Research is being conducted on analytical modeling of nonlinear aspects of molten metal droplet formation and transfer, and integration of knowledge-based control methods (including artificial neural networks and fuzzy logic based connectionist systems) with iterative learning control methods. Results are being transferred to industrial parthers through a related EE-OTT CRADA on Intelligent Diagnostics, Sensing, and Control of Thin Section Welding.

New work has been started on control methods for distributed thermal processes. The focus of this work is specifically on processes employing one or more point sources of heat and or mass with spatial rastering and temporal modulation of the source(s) to produce a distributed temperature field in a distributed mass. The prototypical process is plasma hearth melting of metals. The initial work is investigating iterative learning control to control the trajectory of a heat source through state space (including both the spatial terajectory of the heat source and the thermal parameter trajectory).

This project is part of a collaborative research program with the Massachusetts Institute of Technology.

\section{Idaho National Engineering Lab}

Nondestructive Mat'ls Charac Grp $\$ \mathbf{\$ 2 0 5 , 0 0 0}$ Idaho Falls, ID 83415-2209 03-B

\section{Nondestructive Evaluation of}

\section{Superconductors}

K. L. Telschow

This project is concerned with the development and application of new nondestructive evaluation (NDE) techniques and devices for the characterization of materials, particularly high-temperature superconducting materials in tape form. Microstructural and, particularly, superconducting properties, need to be measured noninvasively and spatially in order to aid the fabrication process. 
Two approaches that are both noncontacting and potentially applicable to the industrial environment are-being-investigated separately and together. One approach uses noncontacting induced current for determination of critical currents on-a-local scale. This technique can be used alone or in conjunction with-externail applied fields and DC transport currents to determine-spatial-variations-in critical current density. Its operation is based on inducing the critical state and determining full penetration through the tape with a small probe coil. A new integral equation approach has been found and solved iteratively that determines the flux front profile in geometries with azimuthal symmetry accounting for demagnetization effects. The capability of high temperature SQUID sensors for measurements in long length tapes is being investigated for increased sensitivity and full hysteresis behavior determination. The second approach uses lasers to generate and detect ultrasonic wave modes in tape geometries. Specific elastic wave modes are employed both analytically and experimentally to determine layer thickness, elastic constants and grain orientation. The stability of the critical state to elastic strain is being investigated using both approaches simultaneously-in-a-coupled-mode.

\section{University Of Illinois}

\author{
Coordinated Science Laboratory $\$ \$ 135,000$ \\ Urbana, IL 61801 \\ 03-A \\ 94-3 \\ Model Bullding, Control and Optimization of \\ Large Scale Systems

\section{T. Basar}

This program involves fundamental research on the modeling, control, and optimization of large scale systems. It encompasses both linear and nonlinear models, deterministic and stochastic systems with external and internal uncertainty, systems with weak spatial and weak or strong informational links, and dynamic decision models with multiple criteria. The main theme is goal-oriented model simplification for control and decision making. The overall goal is the development of new and effective methodologies for robust control, stabilization and optimization of large scale systems in the presence of static as well as dynamic uncertainty, and the analysis of such systems using the concepts of multimodeling, decomposition and aggregation.
An area of current research is the derivation of optimally disturbance attenuating adaptive controllers for nonlinear systems with parametric and additive - uncertainty, when full state measurements-are-not-available-Our method-of approach-here-combines-robust-identification and robust control in a novel way, leading to asymptotically-certainty-equivalent controllers with optimum transient behavior. Another topic of study is the optimal and robust control of structurally-changing, large-scale uncertain systems with separable slow and fast dynamics, where the structural changes are stochastic and are modeled by a finite-state Markov jump process, and the additive uncertainties in the system dynamics and measurement channels are deterministic and norm-bounded. This complements our recent other study on similar systems where the Markov chain transitions exhibited a time-scale separation.:

\section{University Of Illinois}

Dept of Chemical Engineering

Urbana, IL 61801

$\$ 150,000$

01-C

Gas-Liquid-FIow-in-Pipelines

\section{T. Hanratty}

Many operations in the petroleum industry involve the flow of gas and liquid in pipelines. Examples are offshore oil platforms and the transportation of natural gas in pipelines. The economic and safe design of these systems is handicapped because of difficulties in predicting how the phases distribute. Considerable progress has been made by relating large scale behavior to small scale interactions between the phases.

Slug flow is a pattern that exists in horizontal flows whereby slugs of highly aerated liquid fiow intermittently along a pipeline. Of critical importance is the prediction when this pattern will exist and the prediction of the frequency of slugging. Usual practice is to examine the stability of a stratified flow, where the liquid flows along the bottom of a horizontal pipe and the gas concurrently above it. A different approach, which involves a study of the stability of slugs, was undertaken this past year. This provided an explanation of the initiation of slugging at high gas velocities and at high pressures. Measurements of the time and spatial variation of the liquid height along a pipeline is providing the physical 
understanding of the formation of slugs needed to predict frequency.

An important configuration which appears in condensers and evaporators, as well as in petroleum pipelines, is annular flow. Part of the liquid flows along the wall and part as drops entrained in the gas. A critical problem in horizontal pipes is the prediction of asymmetries due to gravity. Experiments were designed during the past year to examine the distribution of drops and of the liquid film over the pipe cross section. This work will be particularly important to understanding the performance of large diameter pipes for which the transition from stratified to annular flow occurs over a wide range of gas velocities.

\section{University Of Illinois}

Dept of Mechanical and Industrial Engineerin

$\$ 80,535$

Urbana, IL 61801

$01-\mathrm{A}$

96-3

Origins of Asymmetric Stress-Strain

Response in Phase Transformations

H. Sehitoglu

A number of uniaxial and stress state experiments on the NiTi alloys that are known to undergo thermo-elastic phase transformations were conducted. Unlike steels which exhibit virtually no recoverable transformation strains, the transformation strains in this class of materials are partially recovered upon unloading, depending on the applied strain levels. Using a servohydraulic intensifier, a servohydraulic test machine, and a novel pressurized test chamber; pressures of $750 \mathrm{MPa}$ and axial stresses of almost any magnitude are simultaneously generated and applied to the gage section of a solid, cylindrical NiFi=specimens:- The $=$ work - utilizes $=a=$ robust internal load cell that can measure axial forces without the effect of seal friction and demonstrate innovative ways of calibrating this load cell, and methods of axial ${ }^{-}$and circumferential - strain measurement in a pressure environment and verify accuracy of these results. Constitutive models proposed in the literature for thermo-elastic transformation were evaluated in light of these results. The current models predict that the volume fraction of martensite is solely dependent on the effective stress. Our experimental results indicate that there is a dependence of the transformations strain on the hydrostatic stress component with strong asymmetry in tension versus compression. In view of these experimental findings, new transformation models are being developed incorporating the low symmetry of the twinning planes. The stress-induced phase transformation of CuZnAl was also found to be stress state dependent but less so than NiTi.

\section{Johns Hopkins University}

$\begin{array}{lr}\text { Mechanical Engineering Dept } & \$ 117,708 \\ \text { Baltimore, MD 21218 } & 01-C \\ & 96-3\end{array}$

Two-Fluid Averaged Equations for Multi-

Phase Flow

\section{A. Prosperetti}

The ultimate purpose of this study is to develop accurate averaged-equations models of disperse multi-phase flows of engineering significance.

This work builds on results obtained in the course of the previous DOE grant in the course of which a new method of phase averaging was developed. The current project aims at deriving the structure of the closure terms for the equations by relying on a series of numerical simulations. Particular emphasis is placed on the calculation of terms involving spatial derivatives of the averaged quantities, that are essential for the stability and hyperbolicity of the model.

The challange here is to be able to calculate such terms relying on techniques developed for uniform suspensions. A new approach capable of achieving this end has been developed and is being implemented. 
Robert H. Kraichnan, Inc. 369 Montezuma 108

Santa Fe, NM 87501-2626

$\$ 74,803$

$01-C$

96-3

Turbulence Theory and Reduced

Hydrodynamics

R. Kraichnan

Turbulent flow is omnipresent in geophysics and energy-producing devices. Atmospheric turbulent transport plays an essential role in the movement of heat, moisture, and pollutants. Turbulent flows represent an enormous amount of data. Both for practicable computation and for physical understanding, it is necessary to extract the essential information in compact form. This project explores novel approaches to economical description and computation of turbulence. Explicit statistics have been deduced theoretically for the spottiness of small scales of a contaminant carried by turbulence. This is the first such success for a turbulence problem, and has sparked an intense and continuing international follow-up effort. The theoretical work has been used under this Grant to guide computer simulations of unprecedented resolution. Recent attention has been focussed on intermittent velocity fields. A result of this project should be improved, economical computation of contaminant dispersal and mixing.

\section{Lawrence Berkeley Laboratory}

\section{Dept of Physics}

University of California

Berkeley, CA 94720

\section{Studies in Nonlinear Dynamics}

\section{A. Kaufman, R. Littlejohn}

Our research concerns the development of methods of modern nonlinear dynamics with applications to problems in physical and engineering sciences. We have been especially involved with Hamiltonian dynamical systems and the application of differential geometric and topological methods. A main area of application is wave systems, in which we have taken a broad, interdisciplinary perspective. Our interests have included the propagation, spectra, mode conversion, and tunnelling of waves. Major divisions of the program are: (1) A study of the properties of coupled wave systems, including elastic waves in solids, electromagnetic waves in optical media or plasmas, nuclear wave functions in molecular physics, and many others; (2) The development of a new method for decoupling coupled wave systems, including systematic adiabatic perturbation schemes for this purpose; (3) The development of asymptotic quantization methods for coupled wave systems, i.e., the determination of normal mode frequencies and eigenfunctions; (4) Investigation of differential geometric and topological concepts such as Berry's phase, gauge structures, and topological singularities (such as monopole strings) which generically occur in coupled wave systems; (5) A systematic study of mode conversion (otherwise called Landau-Zener transitions), in which we apply bifurcation and catastrophe theory to categorize the basic types of mode conversion which can occur; (6) Investigations into coherence and radiometry in optics; (7) The development of theories of mode conversion applicable when nonlinear effects are important; and (8) A study of the non-Abelian gauge fields which occur in the separation of rotational and internal coordinates in the $n$-body problem (with applications to celestial mechanics, satellite control, and atomic, molecular, and nuclear physics).

\section{Lawrence Livermore National} Laboratory

Laser Programs, L-482

University of California

$\$ 128,000$

Livermore, CA 94551

06-A

94-1

\section{Steady State and Transient Nucleation \\ Kinetics \\ G. Wilemski}

This research addresses several fundamental issues in homogeneous nucleation theory. The appealing simplicity and wide applicability of classical nucleation theory is offset by its inaccurate predictions of nucleation rates. New molecular theories of nucleation appear capable of providing greatly improved predictions, but practical application of these theories is currently restricted to the simple rare gas systems. One aim of this work is to bridge this gap by developing theoretical expressions for cluster evaporation rates based on computer studies of the energetics and dynamics of small clusters of more complex molecules such as water and methanol. To preserve a modicum of simplicity in the theory, this work will strive to obtain results that depend on only a few readily determined molecular parameters. 
Theoretical work will also be performed to test and improve various aspects of binary nucleation theory. Accurate numerical solutions of the birth-death equations will be obtained to simulate binary nucleation kinetics for fluid systems with strong compositional surface enrichment, partial miscibility, and significant vapor phase nonideality. These results will be used to assess the accuracy and applicability of various approximate analytical expressions for the rate of binary nucleation. Attention will be given to the effects of transient behavior on binary nucleation rates and to cases of ridge crossing, i.e., when the major nucleation flux bypasses the saddle point. To improve understanding of the effects of partial miscibility on nucleation, molecular dynamics and Monte Carlo simulations of binary rare gas clusters will be made to investigate how the internal molecular distribution of the two species varies as the different interaction strengths are varied.

\section{The Lovelace Institutes}

Institute for Basic and Applied

Medical Research

$\$ 50,428$

Albuquerque, NM 87108

03-B

96-3

Two-Phase Flow Measurements by NMR E. Fukushima, S. Altobelli, A. Caprihan

The objective of this grant is to apply NMR to study how mixtures of different phases flow. The concentration profile of one or both of the phases undergoing flow as well as velocity profile and other more esoteric quantities such as acceleration and diffusion can be measured without interfering with the flow. The non-invasive measurement of such parameters is especially difficult for concentrated mixtures that are opaque to the standard measurement medium such as light and sound waves but NMR works very well provided we examine proton containing liquids, e.g., water and oil, in nonmetallic containers.

Steady flows of concentrated suspensions in a circular pipe, first with a constant cross-section and then with a step expansion and contraction, have now been studied. Because NMR imaging is a fairly slow method, it is very important to extend the NMR method to faster flows. As a point of reference, velocity images have been made in tens of minutes whereas the achievement of similar results in fractions of seconds will be a specific objective for the next few years.

\section{University Of Maryland}

Dept of Mechanical Engineering

College Park, MD 20742

Characterization of Metal Cutting Dynamics

\section{B. Berger, I. Minis}

The development of energy efficient machine tools which produce parts of high quality and require minimum intervention requires the capacity to identify precursors of unstable, chatter states and integrate such identifiers into control system algorithms. Large amplitude oscillations associated with chatter adversely effect surface finish, dimensional accuracy and tool wear often resulting in tool breakage.

This research effort has addressed the fundamental issues involved in the identification and control of chatter through: (i) creation of a data base of over 200 cutting experiments comprising $16.10^{7}$ separate measurements for ranges of values of depth of cut, turning speed and feed rate; (ii) analysis of time series in the data base with a variety of techniques including false nearest neighbors, mutual information, singular value decomposition, time-frequency calculation and polyspectral methods; identification in the data analysis of three nondimensional measures of the cutting state which are sufficiently robust for use in the on line control of the cutting machine; (iv) incorporation of the measures of the cutting state into the control system of an existing lathe.

A relationship has been established between the cutting state and the structure of the power spectra of the time series and its envelope. This research has resulted in the development of means for the elimination of chatter in orthogonal cutting.

\section{University Of Maryland}

Dept of Electrical Engineering

College Park, MD 20742

$\$ 100,000$

06-C

96-4

\section{Mathematical Models of Hysteresis \\ I. Mayergoyz}

This research is concerned with nonlinear diffusion of electromagnetic fields in hysteretic media. Examples of such media are ferromagnetic conducting media with magnetic hysteresis and 
superconducting media with sharp or gradual resistive transitions.

the signal's state of polarization and the corresponding - terms-in-the - modified-Manakov equation that describes the complete evolution.

The main research objectives of the project can be briefly_summarized_as_follows:_development_of analytical techniques for the calculation of eddy currents in magnetic media with hysteresis for linear polarizations of magnetic field (scalar problems), analysis of eddy currents in isotropic and anisotropic media with hysteresis for vector polarizations of magnetic field (vector problems), further development of mathematical models for hysteresis in superconductors with gradual and sharp resistive transitions, development of nonlinear impedance type boundary conditions for hysteretic media and their finite element implementation. It is hoped that, as a result of this research, new analysis techniques will be developed which will find numerous applications in such areas as nondestructive testing, magnetic recording, design of superconducting magnets, induction heating, electromagnetic shielding, evaluation of power losses, etc.

\section{University Of Maryland}

Electrical Engineering Department $\$ \mathbf{\$ 2 0 0 , 4 9 3}$

Baltimore, MD 21228

03-B

$96-3$

Pulse Propagation in Inhomogeneous

Optical Waveguides

\section{c. Menyuk}

We are presently working on two principal projects. First, we are studying randomly varying birefringence in optical fibers and its impact on both soliton and NRZ communications. We have derived a set of equations (modified Manakov equations) that allow us to simulate the propagation through a fiber with rapidly and randomly varying birefringence on the much longer length scale on which the signals varying due to chromatic dispersion, polarization mode dispersion, and nonlinearity. These equations also yield considerable physical insight into the behavior of these systems. We have benchmarked these codes carefully, and we have demonstrated that they yield the same results as computer codes that use far shorter step sizes and are far less efficient. In addition to Monte Carlo methods, we are now using analytical methods based on the theory of stochastic differential equations to completely characterize the probability distribution functions for the evolution of
The-second-project-is-quasi-phase-matched waveguides. We are using a Green's function approach to determine the rate at which radiation leaks from the quasi-phase-matched guides. In the future we will look at oblique guides and guides with other unusual cross-sections that appear in the experiments to reduce unwanted Bragg reflections.

\section{University Of Maryland}

Dept of Mechanical Engineering College Park, MD 20742

$\$ 140,000$

$01-\mathrm{C}$

$94-3$

Contaminant Dispersal in Bounded

Turbulent Shear Flows

J. Wallace, P. Bernard, L. Ong

The purpose of this project is to better understand the physics of scalar transport and to develop a methodology for the accurate prediction of scalar fields in highly sheared environments, including the development of a new class of models that more accurately mimic the dynamical processes affecting plume development. Of particular interest is the wall-normal diffusion of scalar and its connection to vortical structures in the wall region, and the efficacy of the transport models in both canonical boundary layer flows as well as in complex flow geometries with significant flow separation. Towards these objectives, closely coordinated direct numerical simulations (DNS) and windtunnel experiments of evolving linesource plumes are being conducted.

Simultaneous flow visualization (using a laser lightsheet tomographic system) and velocity measurements-(using a 4-sensor-hot-wire-probe) had been performed in a turbulent boundary layer. A smoke plume seeped into the flow from a wall slot in the windtunnel idealizes the dispersion of a passive scalar pollutant released from a line source in the atmospheric surface layer. These experiments have yielded detailed concentration maps as well as directly measured profiles of the concentration fluxes, and. Preliminary analysis of these results, which will provide a database for model development and verification, indicates good agreement with the DNS as well as the transport models being developed. Quasi three- 
dimensional flow visualizations are being conducted with the light sheets oriented in the spanwise direction in order provide better resolved images for the study of the three-dimensional flow structures.

\section{Massachusetts Institute Of Technology}

The Energy Laboratory

Cambridge, MA 02139

$\$ 124,000$

03-B

$94-3$

Metal Transfer in Gas Metal Arc Welding

T. Eagar, J. Lang

Three projects have been undertaken, all aimed at improved control of the final properties of a weld.

The first project, now completed, was a study to model droplet detachment dynamics. Experimental data was generated using a specially developed GMAW system with laser imaging, high speed video, and electrode vibration mechanics. Simulations based on a lumped parameter model were also conducted and good results with the experiments attained.

The second project is to develop a semitransferred plasma welding system. This system is presently under construction. It will consist of two independent plasmas. A transferred plasma is used for substrate heating, while a second non-transferred plasma is used to provide a spray coating stream. Each will be independently controlled with a separate power supply.

The third project is to model and predict the physics of the weld pool during GMAW. The first phase of the experimental component of this project has been completed. The theoretical part is currently under way. Present efforts are focused on determining the shape of the free surface of the molten metal and its influence in the fluid flow, and the influence of Marangoni flows due to compositional differences between the impinging droplet and the substrate.
Massachusetts Institute Of

Technology

Department of Chemical Engineering $\$ 116,000$

Cambridge, MA 02139 03-A

94-3

Synthesis and Optimization of Integrated

Chemical Processes

L. Evans, P. Barton

The goal of this research program is to develop new systematic methods for the synthesis and optimization of chemical processes. As the chemical industry is one of the largest consumers of energy in the US, it is important to find efficient and creative computer-aided design strategies for developing new manufacturing processes, and retrofitting existing plants.

Research is currently being conducted in two related thrust areas. The first thrust is addressing the need for process design and optimization technologies for batch/semi-continuous processes, which are increasingly important as the US shifts to the production of smaller quantities of high value added products. Research is focusing on design tools for rapid and efficient process development. Our approach is based on the development of simplified models that can rapidly calculate a rigorous lower bound on the operating costs of a new process. We are also exploring how our-simplified models -can-be-coupled - with rigorous dynamic models to derive the first rigorous approach that can cope with both discrete and continuous decisions in the optimization of a batch process design.

The second thrust is exploring algorithmic approaches to the optimization of dynamic systems with path constraints. Such an optimization technology could be applied, for example, to determine optimal operating policies for batch unit operations, or optimal feedstock changeovers in oil refineries. Our approach combines theoretical insights into the properties of differential-algebraic equations with advances in both numerical and symbolic computing to yield a novel, rigorous and efficient approach to this class of problems. 


\section{Massachusetts Institute Of Technology}

The Energy Laboratory

Cambridge, MA 02139

$\$ 153,000$

03-B

94-3

Multivariable Control of The Gas-Metal Arc

Welding_Process

D. Hardt

Continuing from last year we have been pursuing three related topics: development of a unique high bandwidth are - furnace, development of a "variable footprint" welding torch, and exploration of distributed parameter models, sensors and controllers. These topics are all motivated by the need to have greater control over the spatial distributions, owing to the limitations imposed by a lumped parameter modeling approach. The arc furnace work was completed this year, with demonstration of de-coupled temperature and flowrate control. A US patent has been issued for this furnace concept and the attendant control system.

The work on the variable footprint torch is pursuing a Gas-Modulated Plasma Arc approach. Characteristics of the new hardware include decoupled heat and filler metal delivery, variable heat output distribution and modular construction for multi-functionality. A physically-based model is currently under development as an aid in designing an appropriate controller for the said torch. The model will be tested and verified upon completion of the torch, currently under in-house fabrication.

In the area of distributed parameter control, we are considering both the basic modeling form along with a multivariable optimal control philosophy. Techniques are being developed for optimally locating and shaping (in space and time) heating/cooling sources (e.g., cooling passages in a mold). The theory for optimal location of measurements has been studied, and simulations and experiments were conducted to study the findings. As an example application, transient temperature control was implemented on a model used by Bethlehem Steel Corporation for a hot slab mill. The techniques being developed are being used as guidelines for developing new actuators (heaters, torches etc.) and sensors for a variety of industrial processes.

\section{Massachusetts Institute Of} Technology

National Magnet Laboratory

Cambridge, MA 02139

$\$ 89,068$

01-D

95-3

Cryotribology (Low-Temperature Friction and

Wear):-Development-of-Cryotribological-

Theories and Application to Cryogenic

Devices

\section{Y. Iwasa, E. Rabinowicz}

To advance our understanding of cryogenictemperature sliding stability, and thereby to improve the reliability of superconducting magnets, we have been examining, experimentally and theoretically, the fundamental mechanisms of frictionally stability. The attainment of absolutely stable, positive friction-velocity characteristics at cryogenic temperatures appears improbable because of the lack of thermally-activated steadystate shear creep. We are presently investigating: 1) a force-based approach to magnet design that promotes quench-causing conductor microslips to occur early in the magnet's charging cycle where their consequences are relatively benign; and 2) the cryotribological behaviors at $77 \mathrm{~K}$ and $4.2 \mathrm{~K}$ of several metal/metal and other nonpolymeric sliding pairs, particularly of several hard, creep-resistant, chemically inert materials such as: the Group 8 noble metals, high-strength ceramics and recentlydeveloped sputter-deposited diamond films. Of particular interest is the extent to which hardness, ductility, and chemical compatibility influence cryogenic-temperature sliding behavior.

\section{Massachusetts Institute Of Technology}

The Energy Laboratory $\$ \$ 192,000$

Cambridge, MA 02139 01-A

94-3

Modeling and Analysis of Surface Cracks D. Parks, F. McClintock

We are developing a mechanics basis for analyzing the fracture behavior of cracks located on or near the fusion zones of structural weldments. Such welds are often characterized by significant strength mismatch between base plate and weld metal, as well as by local strength gradients associated with metallurgical details of the heat-affected zones. Moreover, the local gradients in microstructure, and the accompanying 
gradients in material resistance to both ductile hole growth and cleavage fracture mechanisms provide additional complexity, compared to the corresponding fracture mechanics models of macroscopically homogeneous crack-tip microstructures and properties.

Under macroscopic mode I loading, strengthmismatched interface crack-tip stress and deformation fields show considerable differences from the corresponding fields in mechanically homogeneous media. In particular, both triaxial stress and plastic strain levels in the softer domain (e.g., an undermatched baseplate) are elevated. Families of mismatched fields have been characterized by finite element and slip-line solutions, and have been shown to apply from small-scale yielding through fully-plastic conditions.

The mismatched fields are being coupled with local models of cleavage and ductile fracture in the inhomogeneous crack-tip region, and the results compared with experiments on both model weldments created by diffusion-bonding and with actual welds in A710 steel.

\section{Massachusetts Institute Of Technology}

Dept of Chemical Engineering $\$ 162,554$ Cambridge, MA 02139

Development of Principles \& Methodologies of Metabolic Engineering

\section{G. Stephanopoulos}

Metabolic Engineering is an emerging field of biotechnology aiming at the directed modification of the metabolic pathways of microorganisms, plants and animals using recombinant DNA technology. The overall objective is to achieve overproduction of fuels, chemicals and materials, or biosynthesis of novel products through the amplification of selected biochemical reactions or the introduction of new biosynthetic pathways in metabolic networks. The experimental techniques of metabolic engineering are derived from applied molecular biology and are well advanced. However, interactions of metabolic pathways and the general principles governing metabolic fluxes in-vivo are poorly understood. The goal of this research is to contribute to the development of the tools and principles that elucidate the control of flux in metabolic networks. As the problem of determining flux control distributions at the individual reaction level is too complex and experimentally intractable, our approach has examined the control of flux exercised by groups of reactions. Concepts from metabolic control analysis have been extended to groups of reactions in order to identify the group exercising the strongest degree of control on the production flux. The approach is then repeated within the group until single reactions are identified that are of particular importance to the flux of product formation. This approach has been applied with success to the production of aromatic and aspartate aminoacids in Corynebacterium and yeasts.

\section{Massachusetts Institute of Technology ${ }^{1}$}

Dept of Chemical Engineering $\$ \$ 80,000$

Cambridge, MA 02139 06-C

Los Alamos National Lab ${ }^{2}$

MEE-9

95-3

Los Alamos, NM 87545

$\$ 80,000$

$06-\mathrm{C}$

Sandia National Laboratories ${ }^{3}$

95-3

Engineering Sciences Center

Albuquerque, NM 87008-0834

$\$ 80,000$

$06-\mathrm{C}$

Macrostatistical Hydrodynamics

H. Brenner', A. Graham ${ }^{2}$, L. Mondy

Experimental, analytical, and numerical studies of falling-ball 'tracer' particle dynamics in concentrated suspensions composed of dispersed, neutrally-buoyant spheres and rods comparable in size to the tracer have been continued and extended. Falling ball viscometry was shown to be a useful technique to measure the apparent viscosity of mixtures of particles of various shapes and sizes. When two types of suspended particles (such as spheres and rods) are of disparate sizes, the measured apparent viscosity of the homogeneous mixture could be described successfully by a model which approximated the mixture as one of the fraction of larger particles suspended in a hypothetical suspending continuum with a viscosity identical to the effective viscosity of a suspension of the fraction of smaller particles alone. The pressure 'drop' across a ball falling through a quiescent suspension, which 
constitutes another useful dynamical suspension parameter, was measured and, in moderately concentrated suspensions, found to agree well with theoretical results for the comparable pressure drop in a homogeneous Newtonian liquid. For a ball falling in a highly concentrated suspension, the pressure drop seems to be a sensitive measure of phase slip at the containing cylinder walls. Also, spinning-ball rheometry in quiescent suspensions has been developed as a useful adjunct to the falling-ball theometric studies. This technique provides a sensitive measure of phase slip at the spinning ball's surface, as well as another experimental benchmark for ongoing theoretical investigations of slip in disperse systems. Finally, in collaboration with The Lovelace Institutes, nuclear magnetic resonance imaging is being used to study flow-induced particle segregation ('hydrodynamic diffusion') in pipe flow. These data in inhomogeneous flows and complementary video imaging of individual tracer particles in homogeneous flows will provide much needed information on the effects of flow on particle interactions and effective rheological properties at the macroscale.

\section{University Of Minnesota}

Dept of Mechanical Engineering Minneapolis, MN 55455

Heat/Mass Transfer Enhancement in Separated and Vortex Flows

\section{R. Goldstein}

The flow and heat/mass transfer around single short-step and uniform diameter circular cylinders are being studied. Flow visualization indicates that a short step diameter circular cylinder has a much more complex vortex system than a uniform diameter circular cylinder due to vortices formed on the steps of the cylinders. Velocity and turbulence measurements reveal that the interaction between the vortices formed on the steps of the cylinder and those formed near the endwall junction change the vortex shedding frequencies (Strouhal Number) in the wake flow. The measured local heat/mass transfer compares well with flow visualization and velocity measurements, and confirms the vortex flow patterns.

Energy (total temperature) separation associated with unsteady pressure fluctuations, induced by the convective movement of vortices has proved to have strong effects on heat transfer. Energy and total pressure separation in and around a free circular jet, including the effects of acoustic excitation, and the energy (total temperature) separation around a circular cylinder in high speed flow are being investigated. For the flow in the vortex ring structure around a jet, energy separation and the total pressure separation amplitude are greatly increased by acoustic excitation. This excitation is expected to have a strong effect on the jet impingement heat/mass which will be investigated. For the circular cylinders, the preliminary results show that the dominant transient recovery temperature frequency on the cylinder surface in the wake region matches the vortex shedding frequency.

\section{University Of Minnesota}

\section{Dept of Aero Eng \& Mechanics \\ Minneapolis, MN 55455 \\ $\$ 115,000$ \\ $01-\mathrm{C}$ \\ 93-5 \\ Lubricated Transport of Viscous Materials \\ D. Joseph}

The project has as its broad aim the understanding and technological development of lubricated pipelines of heavy oils in core annular flow, and the spontaneous lubrication of oil/water and water/oil emulsions and for slurry transport. The scientific approach relies heavily on experiments and direct numerical simulations. Analytically the problem involves the study of two-phase flows and deals with migration and segregation of lubricating fluid at the wall. The role of inertia in centering density matched core-annular flows and in levitating these flows off the wall when the fluids have different densities has been clarified in recent works sponsored under this grant. Experiments on the effects of different linings and surfactants on the fouling of pipe walls with hydrocarbons were carried out. A patent for a "method of preventing fouling of pipe walls for lubricated transport" was obtained for cement linings (U.S. Patent No. 5,385,175). Another patent for a non-fouling pressure tap is under consideration. It has been found that oil-in-water emulsions used as a coal substitute fluid and stable water-in-oil emulsions used in the processing of synthetic crude oils will lubricate when flow speed is above a critical one and other attainable conditions are satisfied. 
University Of Minnesota

Dept of Mechanical Engineering

Minneapolis, MN 55455

$\$ 157,975$

06-C

$96-4$

Thermal Plasma Chemical Vapor Deposition

of Advanced Materials

J. Heberlein

The objectives of this program include the characterization of plasma reactors used for materials processing in particular for the deposition of diamond films and the generation of ultrafine particles.

For characterizing a particular diamond deposition reactor, a realistic model has been developed for liquid precursor injection into the plasma in front of the substrate. This three-dimensional model is based on a fluid dynamic description of the plasma jet and the injection gas streams, an energy transfer model including evaporation of the droplets, dissociation of the vapors, and recombination reactions according to chemical kinetics. A surface kinetics model describes the diamond film growth. Initial results show reasonable agreement with experiments.

The theoretical description of if reactors for ultrafine powder production has been completed, and temperature and velocity profiles for different reactor configurations and operating conditions provide a basis for future optimal reactor design.

In order to meet needs for spatially and temporally resolved measurements of the characteristics of turbulent plasma jets, a diagnostic capability has been established based on laser scattering techniques. Results of these measurements will be compared with findings obtained at INEL.

For determining transport coefficients of gas mixtures at plasma temperatures, the influence of different interaction potentials during binary collisions has been established and recommendations have been made for potentials providing the most reliable data.
National Academy of SciencesNational Academy of Eng National Research Council

Washington, DC $20418 \quad \$ 1,167,500$

$06-\mathrm{C}$

Department of Energy Integrated 96-2

Manufacturing Fellowship Program

T. Rozzell

Thirty-six three-year predoctoral fellowships in integrated manufacturing are in place administered by the National Research Council, under the aegis of the National Academy of Engineering, following national competitions. The objectives of the program are to create a pool of PhD's trained in the integrated approach to manufacturing, to promote academic interest in the field, and to attract talented professionals to this challenging area of engineering.

The fellowship program was conceived as one response to the loss of competitiveness of the United States in manufacturing. Two related aspects of the problem are the traditional separation of the product design function from the manufacturing function and the lack of an appreciation for the process of manufacturing as an integrated system.

It is expected that the improved manufacturing methods which this fellowship aims to bring about will contribute to improved energy efficiency, to better utilization of scarce resources, and to less degradation of the environment.

\section{National Aeronautics and Space Adminstration} 600 Independence Avenue, S.W. $\$ \$ 162,000$ Washington, DC 20546 06-C 94-5

Center for Aerospace Research \& Education for Minority Students at Southern University I. Blankson

Pursuant to an agreement between the Secretary of Energy and the Administrator of NASA, the recommended funds will support the education of 
minority students at Southern University (an $\mathrm{HBCU}$ ) in disciplines related to aeronautics and space sciences. Over the five years of this agreement, NASA will provide $\$ 2,500,000$. The education program will be coordinated with a research program addressing Solid mechanics and Finite element Modeling, Composite materials, Aerodynamics and impact, as well as Thermal sciences (Heat transfer and Fluid Dynamics). In more detail examples of specific projects are Improvement of satellite rendezvous maneuvers, optimization of gear design, analysis of the wear of diamond tools. Furthermore, these funds will be used to strengthen the undergraduate education in aeronautics at Southern University.

\section{National Center for Manufacturing Sciences}

Technology Sourcing

Ann Arbor, MI 48108

\section{Industrial Liaison Pilot Program} J. Sheridan

The National Center for Manufacturing Sciences has been funded by DOE, Basic Energy Sciences, to direct a progream which will place employees of the National Laboratories at Industrial sites to work with their corporate colleagues on issues of importance to manufacturing industry. The goals of the program are to evolve expertise in a broad range of manufacturing issues at the National laboratories, identify fundamental DOE research needs, and build a long-range sustained commitment to collaboration on industrial issues both at DOE and within industry. Results from the Liaison Program will strengthen industry's energy efficiency in its operations by enhancing the qulaity of the total product and developing efficient production and management techniques. This will directly benefit American manufacturing competitiveness and will help DOE Basic Energy Sciences identify fundamental research needs in energy efficient industrial methodologies and technologies.

A pilot program will be conducted to immediately place a limited number of workers and carefully evaluate the effectiveness of a sustained program.

The pilot program is important. There are a number of potential risks and blockers which need to be assessed carefully before a large commitment is made. The Industrial Liaison Program (ILP) will place DOE laboratory workers into the industrial environment to focus the attention of the laboratories on industry issues. The worker will remain a full time employee of the Laboratory but the costs of the off-site per diem will be shared by the host company through a formula-based reimbursement.

\section{National Institute Of Standards \& Technology Thermophysics Division Boulder, CO 80303 \\ $\$ 127,500$ 06-A \\ 94-3}

Gelation of Dense Silica Suspensions: Effect of Shear H.J.M. Hanley

The project examines the gel structure of silica by SANS (small angle neutron scattering) and light scattering measurements. The goal is to extend the current understanding of how gels form on a microscopic scale, and to determine what specific changes are induced in the microscopic structure by the influence of an applied shear. Scattered intensity data were taken on the $30 \mathrm{~m}$ SANS instruments at the NIST Cold Neutron Research Facility. Samples were aqueous gels of Ludox silica particles of nominal diameter $24 \mathrm{~nm}$. The series of experiments were repeated with the silica samples loaded into the NIST Couette shearing cell. The results showed that shear most conclusively influences the structure of the gel. On the assumption that an investigation of decomposition of a system to a solid would lead to an insight into the gelation mechanism, a simulation of a two dimensional Lennard-Jones system was initiated. A striking similarity between the computer results and the SANS data was observed. A scaling relation for the time evolution of the structure factor of a decomposing system was proposed. SANS experiments carried out of $7 \mathrm{~nm}$ silica gels have verified that the scaling relation applies to the gelling system. Light scattering experiments on multiply scattering from the silica systems are in progress. 


\section{National Institute Of Standards}

\& Technology

Thermophysics Division

Gaithersburg, MD 20899

$\$ 425,000$

Boulder, CO 80303

$06-\mathrm{C}$

96-3

Development of Measurement Capabilities for the Thermophysical Properties of EnergyRelated Fluids

\section{R. Kayser, W. Haynes}

The major objectives of this new three-year project are to develop state-of-the-art experimental apparatus for measuring the thermophysical properties of a wide range of fluids and fluid mixtures important to the energy, chemical, and energy-related industries. The specific measurement capabilities to be developed are the following: Small-Volume, Dual-Cell Dew-Bubble Point Apparatus; Heat-of-Vaporization Calorimeter and Effusion Cell for Vapor-Pressure Determinations; Solubility Measurements Using Magnetic Levitation; Thermal Diffusivity from Light Scattering; and Phase-Equilibria Apparatus for Azeotropic Aqueous-Organic-Salt Mixtures. These new apparatus will extend significantly the state of the art for properties measurements and make it possible to study a wide range of complex fluid systems (e.g., highly involatile, very insoluble, highly polar, electrically conducting, reacting) under conditions which have been previously inaccessible.

\section{National Institute Of Standards \& Technology}

Electromagnetic Technology Div $\$ \$ 116,800$

Boulder, CO 80303

06-C

94-3

High- $T_{c}$ Superconductor-Semiconductor Integration and Contact Technology J. Moreland, J. Ekin

The purpose of this project is to study materials problems faced in integrating high-T。 superconductor (HTS) thin-film technology with conventional semiconducting technologies. The emphasis of the research is to investigate HTSsemiconductor contact systems and novel HTSsemiconductor devices. The ultimate goal is to develop HTS thin-film technology to its fullest potential for multi chip module interconnections, future ULSI source and drain connections, and microelectronic microwave filters. These potential applications provide the motivation for a thorough investigation of HTS thin-film -materials development of these hybrid systems.

Determining the compatibility of HTS thin-film deposition and patterning processing with that of standard Si processing is crucial for expanding the applications of these hybrid technologies.

The nanostuctural properties of HTS materials have proven to have a principal influence on the electrical properties of HTS materials and devices. For this reason the use of scanned probe microscopies are being emphasized for evaluating HTS-semiconductor epitaxy as well as electrical conduction in interconnects and contacts to hybrid device structures. The further development of scanned probe microscopies, specifically for electronic device imaging will be invaluable not only for the HTS-semiconductor integration studies but for all developments in microelectronics in the foreseeable future. The current emphasis is on developing scanning potentiometry based on atomic force microscopy with resolution and sensitivity levels better than $50 \mathrm{~nm}$ and $1 \mathrm{mV}$, respectively. Also, investigations regarding adapting scanning potentiometry for high frequency applications up to $100 \mathrm{GHz}$ are under way.

\section{The City University Of New York}

The City College $\$ 277,092$

The Benjamin Levich Institute 06-C for Physico-Chemical Hydrodynamics $\quad 95-4$ New York, NY 10031

\section{The Rheology of Concentrated Suspensions}

\section{A. Acrivos}

This research program aims to investigate the flow of concentrated suspensions of non-colloidal particles from the fundamental point of view. Earlier studies by the principal investigator and his associates have shown that the rheology of such systems is strongly affected by the shear-induced migration of particles form regions of high shear to low and from regions of high particle concentrations to low which, by distorting the particle concentration profile, can lead to an erroneous interpretation of the experimental measurements pertaining to the effective viscosity of such systems. This shear-induced particle diffusion is also responsible for the phenomenon 
of viscous resuspension whereby, in the presence of shear flow, a settled bed of heavy particles can resuspend even under conditions of vanishingly small Reynolds numbers.

A theoretical model of shear-induced particle diffusion, which was developed earlier by the principal investigator was tested experimentally in various unidirectional flows by measuring the extent of viscous resuspension and comparing it to the theoretical predictions. Very good agreement was found between theory and experiments even in the case of flow in a tube where strong secondary flows are generated owing to the presence of a non-axisymmetric particle concentration profile. In addition, a technique was developed, using LDA, for measuring the particle velocities in concentrated suspensions. This technique has been extended to measure instantaneous 3-d particle velocities and velocity fluctuations in concentrated suspensions undergoing a variety of shear flows in order to further test the predictions of the theoretical model. Theory and experiments are also being done for bidisperse suspensions.

\section{The City University Of New York The City College \\ Dept of Chemical Engineering \\ New York, NY 10031 \\ $\$ 134,255$ \\ 03-A \\ 94-3 \\ Partial Control of Complex Processing \\ Systems \\ R. Shinnar, I, Rinard}

There are two research objectives. One is to understand the control of Fluidized Catalytic Crackers (FCC's), an important goal in itself. The second is to use the FCC as an example to learn about the control of complex chemical plants in which the number of variables to be maintained within acceptable limits exceeds the number of manipulatable variables available for control. This is known as partial control.

Work finished and published this year focuses on the choice of the control system structure. Some important conclusions with regard to both the FCC and many other systems are:

(1) One cannot judge the suitability of any control structure solely on the basis of the performance of the measured variables used for the set points, but must look at the behavior of all the variables that enter the control specifications.

(2) A control structure suitable in one domain of operation (e.g., partial combustion for the FCC) may be totally unsuitable in another (total combustion).

(3) In the FCC linear algorithms are satisfactory for tuning control loops. The main impact of nonlinearity is in the choice of the primary structure itself and realizable domain of set points.

Most recently the impact of design, in this case the creation of additional manipulated variables, has been assessed with respect to partial control. Publication is due shortly.

\section{The City University Of New York The City College \\ The Benjamin Levich Institute New York, NY 10031 \\ 94-3}

Studies in Physico-Chemical Hydrodynamics of Extended Systems

\section{G. Sivashinsky}

The objective of this project is a unified theoretical approach to the description of a variety of physicochemical hydrodynamics systems characterized by a significant disparity between the spatial scales involved. By appropriately performed averaging over short scales one may considerably simplify the original problem, sometimes even lowering its effective dimensionality and thereby making the latter quite tractable either analytically or numerically.

With all their relative simplicity the emerging reduced models proved rich enough to capture many nontrivial features of the original systems which hitherto resisted description by any other means. Specifically, the scale-separation approach will be applied to (1) the mathematical modelling of stabilization, blowoff and flashback of premixed gas flames; (2) the theory of thermal explosion in the reactive premixture subject to the effects of natural or forced convection; (3) the nonlinear dynamics of the interfacial instabilities in multilayered fiows dominated by viscosity effects. 


\section{State University Of New York}

Department of Physics

Stony Brook, NY 11794

$\$ 193,553$

06-C

95-3

Sub-Electron Transfer of Electric Charge in Semiconductor Nanostructure

K.K. Likharev

The goal of the project is to find the crossover between the continuous and discrete transfer of electric charge transfer in semiconductor heterostructures and nanostructures. While the discreteness is well understood in the limiting cases (tunneling and diffusive conduction) the crossover between the two limits has never been followed, though its understanding is of the key importance for the future development of nanoscale electronic devices.

As a result of theoretical effort during the first year of the project, the relation between the charge discreteness and the spectral density of current fluctuations has been established. The spectral density has been calculated for two important models of nanostructures, including one model describing screening. A new high-frequency limit for the density has been found.

On the experimental side, initial magnetotransport measurements of strained-layer Si/SiGe quantum wells have been carried out at $77 \mathrm{~K}$ using a back gate to vary the carrier density of the $2 \mathrm{D}$ gas in the Si well. It has been found that the resulting resistance modulation may be quite substantial (in one example, between $10 \mathrm{kOhms}$ and 200 kOhms), creating favorable conditions for the experimental search for the charge discreteness crossover.

\section{Northwestern University}

Engineering \& Applied Science

Evanston, IL 60208

Thin-Film Characterization and Flaw

\section{Detection}

\section{J. Achenbach}

This work is concerned with the determination of the elastic constants of thin films deposited on substrates, with the measurement of residual stresses in such films and with the detection and characterization of defects in thin film substrate configurations.

There are many present and potential applications of configurations consisting of a thin film deposited on a substrate. Thin films that are deposited to improve the hardness and/or the thermal properties of surfaces are of principal interest in this work. Thin film technology does, however, also include high Tc superconductor films, films for magnetic recording, superlattices and films for band-gap engineering and quantum devices. The studies carried out on this project also have relevance to those applications.

Both the film and the substrate are generally anisotropic. A line-focus acoustic microscope, is being used to measure the speed of wave modes in the thin film/substrate system. This microscope has unique advantages for measurements in anisotropic media. Analytical and numerical techniques are employed to extract the desired information on the thin film from the measured data. Recent results include: (1) use of multiple wave modes to determine thin film constants, (2) measurements of superlattice film constants, and (3) investigation of the effect of surface roughness.

\section{Northwestern University}

Dept of Chemical Engineering

Evanston, IL 60208

$\$ 136,076$

01-B

95-3

\section{Theory of Subcooled Boiling}

\section{S. Bankoff, S. Davis}

Subcooled boiling is perhaps the most efficient means of steady high-heat-flux transfer from solid surfaces, such as in rocket motors and heat treatment of metals. The growth and collapse of small bubbles while attached to the heating surface will be studied analytically and numerically. When the critical heat fiux is exceeded, subcooled film boiling results. The stability of the resulting vapor film, involving momentary contacts or evaporating liquid tongues with the hot solid wall, and the criteria for growth of these contacts determine the minimum film boiling temperature. To begin with, two problems have been considered. The first paper deals with a circular, evaporating liquid wedge, which oscillates in radius, on a hot horizontal plate. This simulates the periodic dryout and rewetting by a thin liquid film under a growing-and-collapsing bubble in subcooled nucleate boiling. The second problem has led to a long-wave evolution equation for a thin vapor film on a horizontal heated plate, in 
subcooled film boiling. Linear stability results when an evaporation number, $E$, exceeds a critical value, $E_{c}$. When $E<E_{c}$, a pitchfork bifurcation is found, and a new evolution branch is found. Work is continuing on both of these problems.

\section{Northwestern University}

Dept of Eng Sci \& App Math

Evanston, IL 60208

$\$ 54,000$

01-C

94-3

Microscopic Interfacial Phenomena During

Flow in Porous Media

M. Miksis

The objective of this work is to study the effect of interfacial phenomena on the motion of a fluid in a porous material. In particular, we will be primarily concerned with the micromechanics of the fluid motion and its effect on the macroscopic flow. On the pore scale, we find a moving boundary problem for the fluid/fluid and the solid/fluid interfaces. During this project period, attention will be directed to understanding and modeling the dynamics of these interfaces. In particular, attention will focus on three specific topics: surfactant effects, motion of an interface into an unsaturated porous medium and breaking phenomena.

We have recently investigated how capillarity affects the dynamics of a foam on the microscopic scale using both analytical and numerical methods. Now we will consider how capillary and surfactants affect the stability and mobility of foam flow in a porous media. Also, we will continue a study on how capillary effects on the microscopic scale affect the motion of an immiscible contaminating liquid in an unsaturated porous material. Finally, we will investigate the dynamics of a fluid interface during breaking. Breaking phenomena occur during the dynamics of twophase flows.

\section{Northwestern University}

Dept of Chemical Engineering

Evanston, IL 60208-3120

$\$ 113,220$

06-C

95-3

Fragmentation and Dispersion of Powdered

Solids in Viscous Liquids

\section{J. Ottino}

Mixing and dispersion of viscous liquids and powders is important in processing applications ranging from polymer processing to the pharmaceutical and aluminum industries. The goal is to conduct broad-based basic work in the area of mixing with the goal of producing useful knowledge to wide array of users.

Subtantial progress was on several fronts. Studies have been completed in the area of aggregation in regular and chaotic flows. Mathematical arguments have been used to describe the initial growth of average cluster size and polydispersity (Hansen and Ottino 1996a). Work on fragmentation including erosion - has been completed as well (Hansen and Ottino 1996b). A comprehensive model dealing with liquid-liquid mixing processes involving drop breakup and coalescence is nearing completion as well. These results are being incorporated in a lengthy review (possibly a monograph) to be submitted in the near future (Ottino et al. 1996c).

S. Hansen and J.M. Ottino, Aggregation and Cluster Size Evolution in Non-Homogenous Flows, J. Colloid and Int. Sci., 179, 89-103 (1996a).

S. Hansen and J.M. Ottino, Agglomerate Erosion: A Non-Scaling Solution to the Fragmentation Equation, Phys. Rev. E, 53, 4209-4212 (1996b).

J.M. Ottino, P. de Rousell, S. Hansen, and D.V. Khakhar, Mixing and Dispersion of Viscous Liquids in Powdered Solids, in preparation (1996c)

\section{Northwestern University}

Dept of Eng Sci \& App Math

Evanston, IL 60208

$\$ 69,992$

06-C

95-3

Stability and Dynamics of Spatio-Temporal

\section{Structures}

H. Riecke

The objective of this project is to improve the understanding of systems exhibiting spatio-temporal chaos by investigating paradigmatic cases. The research encompasses fundamental and applied aspects. Fundamental issues to be addressed are the characterization and classification of states which are disordered in space and time. In applications, spatio-temporal chaos arises in systems ranging from fluid flow to lasers and coupled oscillators. For instance, in wide-area lasers and in laser arrays spatio-temporal chaos can reduce the quality of 
the laser beam and can even be detrimental to the device itself. Stabilization and control of the beam requires a detailed understanding of the origin and the nature of the chaotic state.

Using analytical and numerical techniques, a number of paradigmatic systems will be studied: parametrically driven waves of different types with emphasis on the effect of parametric driving on unstable laser beams; the stability and chaotic dynamics of traveling waves as found in experiments on the convection of liquid crystals; the role of heteroclinic cycles in chaos in rotating convection.

Presently, the dynamics of unstable parametrically driven waves is studied with focus on the unexpected behavior of defects in the wave pattern. The surprising phenomenon of spatial localization of the chaotic activity in the one-dimensional case is now well understood. The stability of oblique combinations of traveling waves (traveling rectangles) is studied.

Qualitative agreement with the experimental observations is found. A new mechanism leading to chaotic motion of sinks and sources of waves in one dimension has been identified.

\section{University Of Notre Dame}

Dept of Chemical Engineering

$\$ 79,945$

Notre Dame, IN 46556

Fundamental Study of Long-Short Interfacial Wave Interactions and Application to Flow Regime Development

\section{M.J. McCready}

Knowledge of flow regime is the most important issue in the design and operation of multiphase flow pipelines. "Growth of interfacial waves that are long compared to the depths of the two phases is known to play an important role in regime transition from a stratified gas-liquid flow. Current procedures for predicting regime transitions use linear instability of one-dimensional momentum equations as a transition criterion. However, recent work has shown that the predictions of one-dimensional equations differ significantly from the full differential equations and further, that the development process of long wave disturbances is nonlinear.
In this project experiments and theory are being used to discover the flow parameters that control the growth rate of long wave disturbances. To allow for accurate comparison to theory, the system under study is a light hydrocarbon oil water flow in a wide rectangular channel oriented close to horizontal. The long-wave - short-wave interactions that occur in these systems and can cause accelerated growth of long waves are being examined. Slight variations of the flow angle from horizontal allow a wide variation in the relative growth rates and speeds of long and short waves. Theory employs weakly-nonlinear simplifications of the complete Navier-Stokes equations and boundary conditions.

\section{Oak Ridge National Laboratory}

$\begin{array}{cr}\text { Engineering Physics } & \$ 1,451,000 \\ \text { and Mathematics Division } & 03-\mathrm{C} \\ \text { Oak Ridge, TN } 37831 & 94-3\end{array}$

\section{Center for Engineering Systems Advanced Research (CESAR) \\ J. Barhen, R. Mann, E. Oblow, L. Parker, V. Protopopescu, N. Rao, D. Reister}

The primary mission of the Center for Engineering Systems Advanced Research (CESAR) is to develop, through innovative basic research, a core of excellence in the area of intelligent systems technology. By combining long-range, future-oriented R\&D efforts with near-term, sustained technology transfer to U.S. industry, and with undergraduate, graduate, and post-graduate student training, CESAR strives to enhance U.S. scientific and technological capabilities in strategic areas vital to energy independence, national security, and global competitiveness.

Currently, CESAR's efforts emphasize two basic research areas: computational nonlinear science and cooperating autonomous systems. Intelligent autonomous systems are characterized as systems that integrate perception, reasoning, and action to perform tasks under circumstances that are either insufficiently known in advance or dynamically change during task execution. Perception, reasoning, and action, as well as the integration of these modules into prototype working systems, present outstanding challenges for inter-disciplinary basic research. The Center continues to investigate a wide variety of these issues in the areas of cooperating agents, mobility, 
manipulation, intelligent multi-sensory systems, empirical estimation, and machine learning. Specifically, CESAR's focus is on the intelligent control of mobile robots in the presence of uncertainties and constraints. The objective is to develop original, generally applicable methodologies. Several areas of cooperative systems research must be addressed, including task decomposition, task allocation, achieving coherence amidst distribution of control, resolution of subgoal conflicts, reasoning about activities of other agents, inter-robot communication, and multisensor fusion.

In the area of computational nonlinear science, CESAR pursues the development of fundamental methods, including neural networks, wavelets, stochastic approximation, global optimization, and parallel processing algorithms for solving complex scientific and engineering problems in distributed systems. These systems are most often modeled by partial differential equations and involve remote sensing components. Several powerful methodologies are currently developed, based on a synergistic infusion of original mathematical, algorithmic, and engineering ideas. In particular, CESAR focuses on: (i) computationally efficient neural network learning and classification algorithms; (ii) powerful global optimization algorithms; and (iii) optimal control techniques for solving identification problems.

Technology transfer of CESAR innovations is rapidly growing, particularly to the automotive and petroleum exploration industries.

\section{Pennsylvania State University}

$\begin{array}{lr}\text { Mechanical Engineering Dept } & \$ 96,893 \\ \text { University Park, PA } 16802 & 01-B\end{array}$

\section{Research on Combustion-Driven HVOF Thermal Sprays \\ G. Settles}

The High-Velocity Oxy-Fuel (HVOF) thermal spray process combines the fields of materials, combustion, and gas dynamics. It relies on combustion to melt and propel solid particles at high speeds onto a surface to be coated. The goal of this research is to understand and improve the HVOF deposition of corrosion-resistant coatings, which are important in many energy-related industries. This involves both experimentation and modeling.
HVOF spraygun nozzle design and operating parameters have been found with which to vary the kinetic and thermal energies of the spray particles independently. Through metallographic analysis, the resulting coating properties are now being studied. The ability to do this is apparently unique, with results which are expected to be of direct use to HVOF users. For example, it should be possible to tailor coatings to produce desirable properties such as low porosity, high density, and high corrosion resistance. An early result is that stainless steel particles already molten before impact tend to produce less desirable coatings than solid particles which fuse upon impact due to their kinetic energy.

Results of the research are presented annually at the National Thermal Spray Conference. One Ph.D. has been educated and a second graduate student is currently working on this project.

\section{Princeton University}

Dept of Mech \& Aero Eng

Princeton, NJ 08544

Mechanisms and Enhancements of Flame Stabilization

C. Law

The program aims to gain fundamental understanding of the structure and stabilization mechanisms of premixed and nonpremixed flames through theoretical and experimental investigations. The following major projects were completed during the reporting period.

The scalar and dynamic structure of premixed and nonpremixed counterflow methane/air, propane/air and hydrogen/air flames has been experimentally investigated by using non-intrusive laser-based techniques and computationally simulated with detailed chemistry and transport descriptions. Results show that the scalar structure including the thickness of a freely-propagating or freely-standing premixed flame is insensitive to strain rate variations such that the flame cannot be extinguished by strain alone. On the other hand, the thickness of a nonpremixed flame scales inversely with the square root of the strain rate, implying that it can be extinguished by straining. Experimental studies on the dynamic structure demonstrate the need to allow for the effect of thermophoresis on the motion of the seeding 
particles when using laser Doppler velocimetry in measuring the flow velocity.

The dynamics and geometry of flame surfaces in nonuniform flows have been analytically and computations studied, including the phenomena of stretch, local extinction, cusp formation and broadening, and burning rate augmentation through flame wrinkling.

\section{Princeton University}

Dept of Civil Engineering

$\$ 116,959$

Princeton, NJ 08544

06-A

93-4

Transport Properties of Disordered Porous

Media From The Microstructure

\section{S. Torquato}

This research program is concerned with the quantitative relationship between transport properties of a disordered heterogeneous medium that arise in various energy-related problems (e.g., thermal or electrical conductivity, trapping rate, and the fluid permeability) and its microstructure. in particular, we shall focus our attention on studying the effect of: porosity, spatial distribution of the phase elements, interfacial surface statistics, anisotropy, and size distribution of the phase elements, on the effective properties of models of both unconsolidated media (e.g., soils and packed beds of discrete particles) and consolidated media (e.g., sandstones and sintered materials).

Both theoretical, computer-simulation, and experimental techniques have been employed to quantitatively characterize the microstructure and compute the transport properties of disordered media. Statistical-mechanical theory has been used to obtain n-point distribution functions and to study percolation phenomena in continuum random-media models. For example, the poresize distribution, lineal path function, and the chord-length distribution function have been investigated and computed. This has led to accurate predictions of transport properties of realistic models of isotropic as well as anisotropic heterogeneous media. Cross property relations have been derived. Rigorous relations which link the fluid permeability to length scales obtainable from Nuclear Magnetic Resonance experiments and the effective electrical conductivity have been derived. Moreover, the effective conductivity has been related to the effective elastic moduli. Recently, 3-D images of a sandstone have been obtained using $x$-ray tomographic techniques and statistical corrleation funcations have been extracted from them.

\section{Purdue University}

School of Mechanical Engineering \$0

West Lafayette, IN $47907 \quad 01-C$

93-3

Effect of Forced and Natural Convection

on Solidification of Binary Mixtures

F. Incropera

This study deals with the influence of combined convection mechanisms on the solidification of binary systems. A major accomplishment of research performed to date has been the development and numerical solution of a continuum model, which uses a single set of equations to predict transport phenomena in the liquid, "mushy" (two-phase), and solid regions of the mixture. Calculations have been performed for aqueous salt solutions and/or lead/tin alloys involving forced convection, thermo/solutal natural convection, and/or thermo/diffusocapillary convection. The calculations have revealed a wide variety of rich and robust flow conditions, including important physical features of the solidification process which have been observed experimentally but have heretofore eluded prediction. These features include double-diffusive layering in the melt, development of an irregular liquidus front, remelting of solid, development of flow channels in the mushy region, and the establishment of characteristic macrosegregation patterns (regions of significantly different composition) in the final solid. Theoretical and experimental studies have also revealed means by which macrosegregation may be actively suppressed, as, for example, through the application of a magnetic field or intermittent rotation of the mold. 


\section{Purdue University}

School of Nuclear Engineering

West Lafayette, IN 47907

Interfacial Area and Interfacial Transfer in

Two-Phase Flow

\section{Ishii}

The objective of the research program is to develop instrumentation methods, experimental data base and models for describing the interfacial structure and behaviors of two-phase flows. In terms of the flow structure, the transverse distributions of the local void fraction, interfacial area concentration, fluid particle size and their axial development from the entrance to the exit will be the primary focal point of the experimental research. For the purpose of understanding the dynamic behaviors, the interfacial velocity, fluid particle coalescence and disintegration are studied. The axial changes in the distribution of void fraction and interfacial area give the information on the particle coalescence and disintegration. These are used to quantify the flow regime transitions. The multi-sensor probes are used together with hot-film probes for these measurements. The focus of the modeling effort is to develop an interfacial area transport equation which incorporates the mechanistic models for coalescence and disintegration of fluid particles. This transport equation describes dynamical change of the interfacial structure and replaces the conventional model based on flow regime transition criteria.

\section{Purdue University}

School of Mechanical Engineering $\$ 124,813$

West Lafayette, IN 47907

01-B

$96-4$

\section{Critical Heat Flux in Micro-Channel Flow \\ I. Mudawar}

This project targets the development of a theoretical model for critical heat flux (CHF) from long heated walls in vertical flow. Flow boiling pattern is examined with the aid of photomicrographic and high-speed video imaging techniques in order to determine the CHF trigger mechanism. Close-up ștudies of the wall region have revealed features common to most bulk flow conditions. At fluxes below CHF, the vapor coalesces into a wavy layer which permits wetting only in wetting fronts, portions of the liquid-vapor interface which contract the wall as a result of the interfacial waviness. These waves are generated from the heater's upstream region with wavelengths following predictions based upon the Kelvin-Helmholtz instability criterion. Critical heat flux occurs when the pressure force exerted upon the interface due to interfacial curvature, which preserves interfacial contact with the wall prior to $\mathrm{CHF}$, is overcome by the momentum of vapor at the site of the first wetting front, causing the interface to lift away from the wall. Recent studies have shown this interfacial lift-off criterion facilitates accurate theoretical modeling of CHF in both straight and curved channels. Present studies are focused on extending this model to highly subcooled conditions.

\section{Rensselaer Polytechnic Institute Dept of Mechanical Engineering, $\quad \$ 149,828$ Aeronautical Eng \& Mechanics 01-A Troy, NY 12180-3590 96-3}

Inelastic Constitutive Equation: Deformation Induced Anistropy and the Behavior at High Homologous Temperature E. Krempl

Using experimental results obtained with computer-controlled, servohydraulic testing machines, continuum mechanics and materials science as backgrounds, constitutive equations (mathematical models of material deformation behavior that are used in stress and life-time analyses) are being developed with emphasis on two aspects: Deformation induced anisotropy for large deformation on the one hand and high homologous temperature on the other. Both areas extend the modeling capability of the previously developed "unified," state variable viscoplasticity theory based on overstress (VBO).

A mathematical framework and a formulation for the representation of deformation induced anisotropy has been developed and this theory is now being applied to rolling of metals. In this case an isotropic metal can be deformed into metal with elastic and inelastic orthotropy. Simulation of this process is underway.

The small strain version of VBO has been extended to high homologous temperature and applied to Alloy $600 \mathrm{H}$ at temperatures above 0.7 . 
The model can simulate the experimentally observed creep and tensile behavior. It is also shown that the transition from the solid to the fluid state can be accomplished easily with VBO. Applications to solder materials for which ambient temperature is a high homologous temperature and an effort to reduce the number of needed constants in the model are underway.

\section{Rensselaer Polytechnic Institute Center for Multiphase Research $\$ \$ 127,000$ Troy, NY 12180-3590$$
01-\mathrm{C}
$$ \\ 94-3 \\ Development of Multidimensional Two-Fluid Modeling Capability \\ R. Lahey, Jr., D. Drew}

The focus of the work is on the forces involved in flows that feature the gas phase in configurations other than spherical bubbles, and how to quantify them. The work is being conducted by Mr. Tagir Nigmatulin, with some assistance from Mr. Francisco Moraga.

We are implementing our models for multiple fields (specifically, continuous liquid, dispersed vapor, continuous vapor and dispersed liquid) into a steady, fully developed numerical code in order to examine whether it will predict flow features that resemble flow regime changes.

The geometrical analysis for the prediction of the interfacial area density has resulted in an approximation for the area in several situations. The generalization from area evolution by Kelvin-Helmholtz instability to nonlinear waves has resulted in the derivation of a model that is capable of describing waves on annular flows and Taylor bubbles in slug flow, the latter being described as travelling waves.

We are also studying the analysis of phenomena related to the nonlinear wave model as it applies to multiphase flow. We are analyzing disturbance waves to see if a nonlinear breaking waves are responsible for entrainment of droplets in film flow and bridging by liquid and consequently the transition from film flow to slug flow. We have shown that travelling waves agree well with the shape of Taylor bubbles.

We have derived the deformation lift on a spherical bubble, a prolate spheroidal bubble, and an oblate spheroidal bubble. Bubble deformation at high Reynolds number is modelled using an instantaneous deformation tensor and the resulting lift force is calculated as a function of the tensor components.

The experiment (not supported by DoE, but related to the confirmation of the results) to measure the lift force on bubbles, droplets and rigid particles moving in a turbulent shear flow has been revised, and is being tested.

\section{Rensselaer Polytechnic Institute \\ Dept of Chemical Engineering $\$ \$ 92,000$ \\ Troy, NY 12180-3590 \\ $01-\mathrm{C}$ \\ $93-4$}

Development and Use of Image Scanning

Ellipsometer to Study the Dynamics of

Heated Thin Liquid Films

P. Wayner, Jr., J. Plawsky

The physicochemical phenomena associated with fluid flow, change-of-phase heat transfer, and drying in ultra-thin (thickness less than $10^{-5} \mathrm{~m}$ ) liquid films are being studied. In these thin liquid film systems, the interfacial, intermolecular, force field, which controls fluid flow and heat transfer, is a function of the film thickness profile. If the physical properties of the evaporating liquid film are known, the film thickness profile, measured experimentally, can be used to calculate the pressure field and the local evaporative heattranșfer rate. To measure the film thickness profile, a novel ellipsometric technique (Image Scanning Ellipsometry, ISE) has been developed. ISE combines classical null ellipsometry with microscopic image processing, to determine simultaneously the thickness of the liquid film at all points on the substrate. Image processing helps identify the precise location of the surface of the liquid film which corresponds to the measured thickness. Thus a 2-D profile of the liquid film can be constructed. Currently, a second generation ISE employing enhanced resolution image acquisition and processing equipment is nearing completion. The augmented processing power of the second generation instrument is expected to help plot more precise thickness profiles. Presently, the study of the formation of thin aerogel films is being emphasized. Results obtained using the initial design were presented in Applied Optics, 33, pp 1223-1229, 1994, and, Physics Fluids, 6, pp 1963-1971, 1994. 


\section{Rice University}

Dept of Civil Engineering

Houston, TX 77251

$\$ 88,000$

$01-A$

95-3

A Novel Nonlinear System Identification Approach with Applicability to Aging of Energy Production and Distribution Systems P. Spanos

Nonlinear forces acting on energy systems such as large offshore structures, are studied from a perspective of system identification. The nonlinearities may be induced by ocean waves, and they may become significant in several situations. They are not necessarily assumed to be in the form of Morison's equation. Various wave forces are examined. The force function is decomposed either into a set of base functions, or it is expanded in terms of the wave and structural kinematics. The resulting nonlinear system is decomposed in a number of parallel non-memory, nonlinear systems, each followed by a finite memory subsystem. A Gram-Schmidt orthonormalization procedure is applied to decouple these subsystems; a frequency domain technique involving auto-spectra and cross-spectra is employed to identify the linear transfer function. In conjunction with this problem, an efficient algorithm for representing random processes by auto-regressive-moving-average digital algorithms is pursued for implementation into system identification problems. The analytical results are calibrated by Monte Carlo simulation studies.

\section{University Of Rochester}

Dept of Physics and Astronomy

Rochester, NY 14627

$\$ 71,930$

$06-\mathrm{C}$

95-3

Flux Flow, Pinning, and Resistive Behavior

in Superconducting Networks

S. Teitel

The fluctuation of vortices and vortex lines has been shown to be a major source of electrical resistance for superconducting networks when placed in magnetic fields. Systems of particular interest include the new high temperature type II superconductors, and periodic arrays of Josephson junctions. Numerical simulations are being carried out to identify and characterize the nature of the various vortex structures present in such systems, as a function of temperature and applied magnetic field, and to understand the nature of the phase transitions between them.
Particular attention has recently been given to studying the equilibrium fluctuation of vortex lines in models of bulk high temperature superconductors.

Simulations have shown that there can be two distinct phase transitions describing the superconducting ordering parallel versus perpendicular to the applied magnetic field. The loss of order in the perpendicular direction has been associated with a melting of the ground state vortex line lattice. The loss of order in the parallel direction has been associated with the onset of a vortex line tangle percolating throughout the entire system. New simulations, relaxing earlier approximations, are being carried out to clarify this issue. The effect of applied currents and random vortex pinning sites will be added in future work. The dynamic behavior of vortices in two dimensional Josephson arrays has also recently been investigated using a detailed finite size analysis to verify proposed scaling equations.

This research will greatly enhance the fundamental understanding of behavior in strongly fluctuating superconducting materials. The results will have impact in understanding the magnetic properties of the new high temperature superconductors, and in the design of Josephson junction arrays for use as microwave detectors and generators.

\section{University Of Rochester}

Dept of Physics and Astronomy

Rochester, NY 14627

$\$ 95,187$

$06-C$

$96-3$

Direct and Inverse Problems in Statistical

Wavefields

E. Wolf

The main objective of this research is to elucidate how coherence properties of sources affect the spatial and the spectral distribution of the energy density and the flux vector throughout the radiated field. The analysis utilizes the techniques of statistical wave theory. Among the main results obtained during the past year was the derivation of some new conservation laws for fluctuating free fields, clarification of distinction between changes in spectra due to source correlation and due to diffraction and dispersion and the introduction of a class of model sources each of which generates a field whose normalized spectrum is the same at every point outside the domain occupied by the 
source. Spectral filtering of fields with strong quantum correlations was also studied and the changes in the correlation characteristics of a field produced by a down converter passing through a strongly dispersive element, such as the FabryPerot cavity was calculated. A generalization of the Talbot effect for propagation in a graded index medium was_also studied.

\section{The Rockefeller University}

Department of Physics

$\$ 93,450$

1230 York Avenue

06-C

New York, NY 10021

$95-4$

\section{Some Basic Research Problems Related to Energy \\ E. Cohen}

1. A very simple interpolation formula for the Newtonian viscosity as well as the visco-elastic behavior of monodisperse hard sphere colloidal suspensions has been derived theoretically for low as well as high concentrations. The formula has been adapted to micelles and is being tested for polymers. An extension to shear-rate dependent effects is in progress.

2. The investigation of Lorentz lattice gas cellular automata is continued. In this gas a point particle moves on the bonds of a lattice fully or partially occupied by deterministic scatterers of two types, which scatter the particle either to its left or to its right and change their scattering character after collision with the particle. Complex diffusion-propagation patterns of motion of the particle then emerge. This complex behavior of a very simple system could well be the prototype for a variety of phenomena found in nature. This is further investigated.

3. Dynamical weights using expanding Lyapunov exponents, rather than the usual probabilistic (Gibbsian) weights, can be employed to obtain the probability distribution of a many particle system in a nonequilibrium stationary state (e.g. a sheared fluid), which can be far from equilibrium. Successful applications of this new approach to obtain the properties of such a system have been made and are further pursued.

\section{Sandia National Laboratories}

Combustion Research Facility $\$ 90,000$ Thermofluids Division

06-B

Livermore, CA 94550

$96-3$

Dynamically Active Scalars in Turbulent Combustion, Heat Transfer, and Geophysical Flows

\section{A. Kerstein}

The principal focus of this research program is modeling of turbulent flows in which mixing modifies flow energetics, as in buoyant stratified turbulence and turbulent combustion. Turbulent motions are represented by mappings applied to a one-dimensional domain on which a velocity profile, and profiles of participating scalar quantities, are defined. The mappings are determined by turbulence energetics based on velocity differences and any dynamically active scalars. The mappings modify the velocity and scalar profiles, yielding a self-contained evolution process.

Molecular transport (viscosity and species diffusion) are implemented concurrently with the mapping process in this fully resolved formulation. The mechanistic distinction between advective and diffusive processes is thus maintained. This distinction is not maintained in models that lack full spatial resolution.

Initial applications to homogeneous turbulence, free shear flows and boundary layers demonstrate that the model reproduces key features of the turbulent cascade and the scaling laws and spatial structure (mean and fluctuation properties) of inhomogeneous flows. In future work, the model will be used to gain mechanistic understanding of turbulent mixing processes involving passive and active scalars, with emphasis on engineering and geophysical mixing processes that have not yet been modeled successfully. 


\section{Science Applications Intl Corp}

Blotechnology Research and

$\$ 299,872$

Applications Group

03-A

McLean, VA 22102

96-3

Function-Based Biosensors for Use in Hazardous Waste Remediation

\section{J. J. Hickman}

Research Objectives and Scientific Approach The goal of this work is to develop a new type of sensor capable of detecting toxins in hazardous waste effluents after remediation. This novel biosensor will monitor the changes in electrical signal between two neurons adhered to a uniquely patterned artificial surface in a defined growth media. The changes in electrical signal will correspond to the changes in neuron-neuron communication upon the introduction of a toxin. This type of sensor would be able to sense known as well as unknown toxins in hazardous waste effluents after remediation.

\section{Current Status of Project}

Great progress has been made in several key areas via the commitment of SAIC internal research and development funds to jump-start and augment this program during the first year. Initial screening established extreme cases of selfassembled monolayer (SAM) modified surfaces which would either promote or impede neuronal growth on the substrates. These SAM's were then used to construct lithographically defined surface for neuronal patterning. A key area of progress was to establish a laser system that gave reproducible patterns. The collaborative effort with NIH, NINDS has allowed the culture and electrophysiological characterization of rat hippocampal neurons on these high resolution patterned surfaces in defined media.

Preliminary cell culture results have indicated we have extended the longevity and fidelity on the patterns. We have applied new surface analysis tools in these investigations that have enabled a clearer understanding of the relationship between surface and cell response. In the second year of the project the primary goal will be extension of the in vitro culture system on the patterns to $>1$ month. Preliminary response of the rudimentary circuits to toxins will also be investigated. Finally, the initial solid-state system will be designed.

\section{Stanford University}

Dept of Mechanical Engineering

Stanford, CA $94305-3030$

Structure and Modelling of the Three-

Dimensional Boundary Layers on a Rotating

Disk

\section{J. Eaton}

The objectives of this work are to develop improved understanding of the effect of mean flow three dimensionality on convective heat transfer through detailed experiments on turbulent transport mechanisms. Experiments have been conducted in two geometries, a large disk rotating in still air and a highly skewed wind-tunnel boundary layer. New miniaturized probes have been developed to measure the turbulent heat flux in the disk boundary layer. The probe uses a cross-wire anemometer with 2.5 micron diameter wires and a separate resistance thermometer. A technique has been developed for frequency compensation of resistance thermometers that allows their use to higher frequencies than previously possible. Temperature profiles have been recorded and heat flux measurements are underway. An extremely high resolution laser-Doppler anemometer system was developed which provides a 35 micron diameter by 60 micron long measurement volume in a laboratory scale wind tunnel. Reynolds stress measurements deep into the sublayer of a skewed boundary layer were acquired using this system. The results showed close alignment of the shear stress and strain rate vectors near the wall. This contradicts previous assumptions that were made in the absence of any reliable data. The remainder of the grant period will be used to complete the heat flux measurements and interpret them in terms of available models.

\section{Stanford University}

Dept of Mechanical Engineering $\$ \$ 137,700$

Stanford, CA $94305-3030$

01-A

95-3

Stress and Stability Analysis of Surface Morphology of Elastic and Piezoelectric Materials

H. Gao, D. Barnett

The objective of this research has been to study morphological stabilities and instabilities in elastic and piezoelectric solid. In morphologies are 
included surface shapes, cracks, and defect patterns. In this past year the conditions for stability or instability of surfaces and interfaces in piezoelectric materials (including arbitrary elastic and piezoelectric anisotropy) have been developed [1]. This work has shown that piezoelectric coupling may tend to either stabilize or destabilize an initial flat boundary or interface. A destabilized surface evolves toward the formation of crack-like flaw. This study suggests that piezoelectric coupling could be utilized to control diffusive initiation of surface defects. A portion of future work will be directed toward corroborating theory with experiments and identifying whether more sophisticated theoretical models for defect generation need to be explored. Another direction which this research has taken is the study of fracture in piezoelectric solids. A strip saturation model and the concept of multiscale energy release rates have been introduced $[2,3]$ to explain some existing experimental observations of the behavior of cracks in piezoelectric ceramics. Extensions of this work are underway.

Patterns of equilibrium 2-dimensional arrangements of large numbers of dislocations have been computed by using numerical methods to minimize the potential energy of the dislocation distributions. Efficiency of computation has been greatly enhanced by studying doubly periodic arrangements of dislocation cells for which some analytic reduction is possible. It has been found that many possible equilibrium patterns exist under zero applied stress, i.e., nearby equilibrium arrangements are always available. A study of the stabilty of these arrays under application of applied stresses is now underway.

[1] N. Y. Chien, H. Gao, G. Herrmann, and D. M. Barnett, "Diffusive Surface Instabilities Induced by Electromechanical Loading", Proceedings of the Royal Society, London, A452, pp. 527-541 (1995).

[2] H. Gao, T.-Y. Zhang, and P. Tong, "Local and Global Energy Release Rates for an Electrically Yielded Crack in Piezoelectric Ceramics", Journal of the Mechanics and Physics of Solids (in review)

[3] H. Gao and D. M. Barnett, "An Invariance Property of Local Energy Release Rates in a Strip Saturation Model of Piezoelectric Fracture", International Journal of Fracture (in review)
Stanford University

Edward L. Ginzton Laboratory

$\$ 231,000$

Stanford, CA 94305-3030

03-B

96-3

Optical Techniques for Characterization of

High Temperature Superconductors

G. Kino

Photothermal techniques are used to measure the normal carrier density below the transition temperature $T_{c}$ in high-temperature superconductors, to study the nature of the phase transition, and to measure the homogeneity and quality of these materials. A modulated focused laser beam incident on the sample varies its temperature periodically, and a second probe beam a few microns away measures the differential reflectivity associated with the thermal wave propagating along the sample. Changes in critical temperature in regions less than $100 \mu \mathrm{m}$ apart have been measured, and the difference in quality of different samples can clearly be seen. Measurement of thermal diffusivity in single-YBCO crystals yields good estimates of the variation of normal electron density with temperature. Observations of small changes in the phase variation yield the transition temperature of the material. Polarized light observations of singlecrystal YBCO near the transition point yield curves as a function of temperature with shapes that are very different, depending on the polarization of the probe beam relative to the $A$ and $B$ directions. Twinned samples do not show this anisotropy. The shape and sign of these curves also appears to provide a very sensitive measurement of the state of doping of the material. By measuring the modulated signals at the second harmonic of the input signal, the temperature modulation of the sample by the laser beam can be determined. During the last year the system has been rebuilt to give more accurate results, to work at lower temperatures so that we can make measurements of normal superconductors and compare with theory, and to make more rapid measurements of the quality of thin film superconductors. 


\section{Stanford University}

Mechanical Engineering Dept

$\$ 235,135$

03-B

96-4

\section{Nonequilibrium Plasma Chemistry C. Kruger, T. Owano}

This research is concerned with optical diagnostics for plasma chemistry and plasma processing, with an emphasis on methods that allow for departures from local thermodynamic equilibrium (such as finite chemical reaction rates, nonequilibrium electron densities and temperatures, and radiation loss effects). Studies in an induction plasma facility show significant nonequilibrium within a downstream reactor, and suggest the possibility of erroneous results when using conventional diagnostics that assume local thermodynamic equilibrium.

Advanced laser-based methods are being developed for measurement of plasma parameters including species concentration and temperature. The primary techniques under investigation are Degenerate Four-Wave Mixing (DFWM) and Cavity Ring-Down Spectroscopy (CRDS). These techniques are being applied to atmospheric pressure and near-atmospheric pressure plasma environments in order to assess the importance of nonequilibrium effects under conditions of interest to plasma chemistry.

Experiments using DFWM to probe $\mathrm{CH}$ and $\mathrm{C}_{2}$ within the reacting plasma have demonstrated the ability to provide sensitive (ppm level) detection with submillimeter spatial resolution in the measurement of vibrational temperatures, rotational temperatures, and species concentrations. The use of CRDS to detect $\mathrm{CH}_{3}$ radicals with high sensitivity and high spatial resolution has also been recently demonstrated.

\section{Stanford University}

Dept of Chemistry

Stanford, CA 94305

$\$ 282,000$

06-C

95-4

Topics in a Thermodynamic and Stochastic

Theory of Nonlinear Processes Far from

Equilibrium

\section{J. Ross}

Research focuses on the thermodynamic and stochastic theory of hydrodynamic processes, such as combinations of chemical reactions, diffusion, thermal conduction, and viscous flow. Such theories have been formulated for each of the individual processes, both linear and nonlinear. Progress has been made on the thermodynamic and stochastic theory of coupled transport processes as exemplified by light-scattering in a fluid in a temperature gradient. The theory has also been extended to study fluctuations near limit cycles in chemical reaction systems and to show the role of Liapunov functions in the issues of stability and relative stability in reaction diffusion systems with multiple stationary states. An extensive analysis has been published on the categorization of some oscillatory enzymatic reactions. In an analysis of glygolytic metabolites by capillary zone electrophoresis the concentrations of eleven different species have been measured simultaneously in preparation for study on the kinetics of this system.

\section{Stanford University}

Dept of Chemistry

Stanford, CA 94305

$\$ 99,017$

03-A

96-3

\section{Laser-Based Diagnostics for Plasma Chemistry \\ R. Zare}

This research continues to pioneer cavity ringdown spectroscopy (CRDS), a new laser absorption technique that provides highly sensitive measurements of absolute concentrations of species in plasmas and other luminous media. Design of a mid-infrared CRDS system that measures $\mathrm{CH}_{3}$ radicals in a plasma torch used for diamond deposition with an optical parametric oscillator (OPO) light source, is underway.

Development of portable and inexpensive CRDS systems that can detect species in different plasmas is in progress. A prototype that uses a commercial laser diode (LD-CRDS) at $830 \mathrm{~nm}$ has successfully measured the spectrum of water vapor in ambient air with a resolution of $0.001 \mathrm{~nm}$ and a sensitivity of $3 \times 10^{-5} \mathrm{~cm}^{-1}$. Based on measurements using the OPO system, LD-CRDS can potentially achieve a sensitivity of $10^{-8} \mathrm{~cm}^{-1}$ while functioning under harsh operating conditions (strong background emission, high temperatures), as illustrated by OPO-based measurements of water vapor in a propane torch flame. 
This research also furthers the understanding of Fabry-Perot theory, which describes the behavior of light inside a ring-down cavity. Extensive modeling tools based on complementary differential equation and a matrix approaches have been implemented. These allow the simulation of cavity output waveforms for optimizing CRDS system parameters.

\section{University Of Texas At Austin}

Ctr for Studies in Statistical Mech

$\$ 99,999$

and Complex Systems

06-C

Austin, TX 78712

94-3

The Behavior of Matter Under Non-

Equilibrium Conditions: Fundamental

Aspects and Applications

I. Prigogine, T. Petrosky

A formulation of the dynamics of chaotic systems and of unstable quantum systems has been developed which includes, in a rigorous manner, the arrow of time on the fundamental level. This formulation unifies thermodynamics and dynamics. This has been done by extending the basic equations of motions (the Liouville-von Neumann equations) in a wider class of function spaces than the usual Hilbert space. This extension applies, in particular, to chaotic maps and to a certain class of unstable Hamiltonian systems, where it yields new solutions on the statistical level that are not implementable in terms of trajectories or wavefunctions. Situations where the generalized formulation applies can easily be compared to numerical simulations. All of these theoretical predictions have been verified in this way.

Some of these predictions are very unexpected, such as the extension of quantum scattering theory for finite times (in contrast with the usual S-matrix approach valid for asymptotic times). This approach led to the discovery of new secular effects which are possible only outside the Hilbert space. Indeed, it was found that a new peak of the transition rate of three-body scattering in momentum space for large wave packets. The peak exists only during the time wave packets are overlapping, and disappears after collision. This discovery offers the possibility of laboratory experiments to observe events occurring outside the usual Hilbert space formulation of quantum mechanics.
In addition, several applications of the this approach are in progress. These include matterfield coupled systems (quantum, optics), nonlinear fields, causal behavior of the soft-core Lorentz gas, and transport processes in moderately dense gas with hard-core interactions and quantum mesoscopic systems

\section{University Of Texas At Austin}

Dept of Physics

$\$ 185,000$

Austin, TX 78712

06-C

93-5

Complex Spatiotemporal Patterns in

Nonequilibrium Systems

\section{H. Swinney}

The formation of time-dependent spatial patterns is being studied in chemical and physical systems driven far from equilibrium. The goal is to understand what features of behavior are common in diverse pattern-forming systems. What kinds of patterns arise spontaneously as the stress is increased on an initially homogeneous system? What kinds of transitions are permitted between different patterns? What is the role of symmetries in the boundary conditions and the equations of motion? These issues and their relation to applications are being addressed in laboratory experiments, numerical simulations, and analyses on reaction-diffusion systems, vertically vibrated granular media, and convecting fluids. A study of rotating spirals in a chemical reaction-diffusion system has revealed a parity-breaking transition from stationary to oscillating chemical spots. Experiments on oscillating granular media have led to the discovery of a new type of excitation: a stable, localized, stationary structure dubbed "oscillon", which is a peak on one cycle and a crater in the next cycle. Experiments and simulations on turbulent convection in a quasi-two-dimensional system, a thin vertical sheet of fluid, show that the behavior is dominated by the dynamics of plumes (birth, growth, merger, and destruction). 


\section{University of Washington}

Dept of Mechanical Engineering

Seattle, WA 98195

$\$ 76,721$

01-A

94-3

3-D Experimental Fracture Analysis

at High Temperatures

A. Kobayashi

The objective of this three year project is to assess experimentally, the validity of $T^{*}$ integral and its applicability to quasi-static and dynamic ductile fracture. Early in the second year, a protocol for extracting the $T^{*}$ integral values from the surface displacement fields obtained by moire interferometry was established. The procedure consists of numerically evaluating the integral along a partial contour, a small distance, $\varepsilon$, in front of the crack tip, In order to assure a state of plane stress, $\varepsilon$ is equated to one plate thickness and the resultant $T^{*}$ is designated $T^{*}{ }_{{ }^{*}}$. The procedure was verified through numerical experiments conducted at the Georgia Institute of Technology (GIT) under a parallel DOE grant.

The established procedure was used to determine $T^{\star}{ }_{s}$ 's of A606 HSLA steel, single-edge notched (SEN) specimens with small stable crack growth, $\approx 2 \mathrm{~mm}$, and 2024-T3 aluminum, compact (CT) of large crack growth, $\approx 8 \mathrm{~mm}$. Parallel numerical analysis of these two sets of experiments were conducted at GIT where the experimentally and numerically determined $T^{\star}{ }_{s}$ were found to be in excellent agreement. $T^{*}{ }_{8}$ of the A606 HSLA SEN specimen continued to increase with stable crack growth, possibly due to the lack of constraint in the SEN specimen. $T^{*}$ of the 2024-T3 CT specimen reached a steady state value of $\approx 140 \mathrm{MPa}-\mathrm{mm}$. The CT specimen results suggest that $T^{*}{ }_{s}$ could be a viable fracture parameter which controls stable crack growth. The crack tip opening angles (CTOA) for the two materials immediately reached steady state values with crack growth. However, results from a FAA funded study showed that CTOA is insensitive to the inherent decrease in ductility due to increased thickness and therefore may not be a proper fracture parameter.
Washington University

Department of Systems Science

and Mathematics

$\$ 91,772$

03-A

Saint Louis, MO 63130

96-3

Visionics: An Integrated Approach to Analysis and Design of Intelligent Machines B. Ghosh

In this project we propose to control intelligent machines that has the ability to operate and perform satisfactorily in an unstructured environment based on visual information. Such a machine has the ability to navigate in the environment, learn about the geometric structure and shape of objects in a partially known environment and to track changes in the position of objects over time. As a specific application, we propose to study the visually guided robotics problem where the system has the ability to autonomously modify the existing planned path in order to avoid an obstacle that has been detected visually.

The role of vision and analysis of dynamical systems based on intelligent machines that can see the environment, has been studied for the last six years. The associated dynamical system has been introduced and studied under the name 'perspective systems' (dynamical systems with perspective observation function). Parameter identification and estimation problems associated with a perspective system has been understood as a course of our investigation. The perspective theory has also been applied to a specific experimental set up which involves visual guidance of a robot manipulator in a manufacturing workcell.

To sum up, building on earlier progress in the field of 'perspective estimation,' this project introduces and studies intelligent machines that are based on 'visually guided estimation and control system design.' The long term goal is to study perspective control systems, that has the ability to perform visually guided autonomous tasks requiring sensor fusion. Overall this project introduces new challenges in control technology with applications to control systems design in remote, unstructured and possibly hazardous environment. 


\section{Washington State University}

Dept of Mech \& Matls Engineering

$\$ 74,304$

Puliman, WA 99164-2920

01-B

94-3

\section{Coupled Particle Dispersion by Three- Dimensional Vortex Structures \\ T. Troutt}

The primary objective of this research program is to obtain understanding concerning the role of three-dimensional vortex structures in the dispersion of particles and droplets in free shear flows. This research program builds on previous studies which focused on the nature of particle dispersion in large scale quasi two-dimensional vortex structures which are a dominant component of free shear flows. Although three dimensional vortex structures can be quite complex in nature, time scaling quantities such as Stokes number can still be expected to be important for understanding the particle dispersion process.

This research program is employing timedependent experimental and numerical techniques to provide information concerning the particulate dispersion process produced by three-dimensional vortex structures. The free shear flows investigated include slightly perturbed plane mixing layers and wakes. Recent three-dimensional twophase flow simulation results indicate that streamwise vortex structures can significantly alter the particle dispersion process in plane mixing layers. The influence of these streamwise structures appears to be most pronounced for particles with intermediate value Stokes numbers.

Intensive experimental and numerical studies of these new observations are presently being pursued. Eventually insights from these threedimensional paricle dispersion results may lead to improvements in the design of practical energy conversion systems.

\section{University Of Wisconsin}

Mechanical Engineering Dept

Milwaukee, WI 53201

Interfacial Area and Interfacial Transfer in Two-Phase Flow Systems

\section{G. Kojasoy}

The objectives of the proposed research program are to develop instrumentation methods, an experimental data base, and an analysis leading to predictive models for describing the interfacial structure and behaviors of horizontal two-phase flows. In terms of the flow structure, the transverse distributions of the local void fraction, interfacial area concentration, fluid particle size and their axial development from the entrance to the exit will be the primary focal point of the research. For the purpose of understanding the dynamic behaviors, the interfacial velocity, wave characteristics, fluid particle coalescence and disintegration will be studied. The axial changes in the distribution of void fraction and interfacial area give the information on the particle coalescence and disintegration. These will be characterized by the collision frequency and interfacial energy and turbulence in the liquid.

A special emphasis will be placed on the further improvement of the multi-sensor resistivity probe method which has been successfully developed and cross-calibrated against other global techniques. The multi-sensor probes will be used together with hot-film probes for the liquid turbulence measurements. These new measurements will give sufficient information to evaluate the local relative velocity and momentum interaction between phases. Final focus of the modeling effort is to develop interfacial area transport equation which incorporates the mechanistic models for coalescence and disintegration of fluid particles. This transport equation describes dynamical change of the interfacial structure and replaces the conventional model based on flow regime transition criteria.

The proposed research program will provide: a) a new scientific instrumentation method for studying detailed interfacial characteristics of two-phase flow, b) benchmark data for the local interfacial area concentration, void fraction distribution, interfacial wave structure, relative velocity and wave propagation velocity for horizontal two-phase flow systems, c) mechanistic models for fluid particle coalescence and disintegration, and d) interfacial area transport equation. 
University Of Wisconsin

Dept of Chemical Engineering

Madison, WI 53706

$\$ 118,960$

03-A

95-3

New Process Modeling, Design and Gontrol Strategies for Energy Efficiency, High Product Quality, and Improved Productivity in the Process Industries W. Ray

The process industries are having great difficulty competing in the world market because of high energy costs, high labor rates, and old technology for many processes. This project is concerned with the development of process design and control strategies for improving energy efficiency, product quality, and productivity in the process industry. In particular, (1) the resilient design and control of chemical reactors, and (2) the operation of complex processing systems, will be investigated. Major emphasis in part (1) will be on two important classes of chemical reactors: polymerization processes and packed bed reactors. In part (2), the main focus will be on developing process identification and control procedures which allow the design of advanced control systems based on limited process information and which will work reliably when process parameters change in an unknown manner. Specific topics to be studied include new process identification procedures, nonlinear controller designs, adaptive control methods, and techniques for distributed parameter systems. Both fundamental and immediately applicable results are expected. The theoretical developments are being tested experimentally on pilot scale equipment in the laboratory. These experiments not only allow improvements in theoretical work, but also represent real life demonstrations of the effectiveness of the methods and of the feasibility of implementing them in an industrial environment. The new techniques developed in this project will be incorporated into computer-aided design packages and disseminated to industry. Therefore, it is expected that the work will have an impact on industrial practice. 


\section{Budget Number Index}

\section{Mechanical Sciences:}

01-A Solid Mechanics: macroscopic aspects of elastic and plastic deformations, and crack propagation

01-B Heat Transfer

01-C Fluid Mechanics

01-D Tribology

\section{Control Systems and Instrumentation:}

03-A Control systems, large scale systems

03-B Instrumentation for hostile environment, and NDE

03-C Intelligent systems

Engineering Data and Analysis:

06-A Thermophysical properties and processes

06-B Combustion

06-C Non-linear dynamics and engineering analysis 
Continuum Damage Mechanics - Critical States ............. 2 An Investigation of History Dependent Damage in Time Dependent

Fracture Mechanics . . . . . . . . . . . . . . . . . . 2

Simulation and Analysis of Dynamic Failure of Ductile Materials . . . . . . . 3

Dynamic Failure Characterization of Ductile Steels $\ldots \ldots \ldots \ldots \ldots \ldots \ldots$

Nonlinear Dynamics of Fluid-Structure Systems . . . . . . . . . . . 11

An Analytical-Numerical Alternating Method for 3-D Inelastic Fracture and

Integrity Analysis of Pressure-Vessels and Piping at Elevated Temperatures . 13

Elastic-Plastic Fracture Analysis Emphasis on Surface Flaws . . . . . . . . 15

Origins of Asymmetric Stress-Strain Response in Phase Transformations . . . 18

Modeling and Analysis of Surface Cracks . . . . . . . . . . . . . 23

Inelastic Constitutive Equation: Deformation Induced Anistropy and the

Behavior at High Homologous Temperature . . . . . . . . . . . . . 35

A Novel Nonlinear System Identification Approach with Applicability to

Aging of Energy Production and Distribution Systems . . . . . . . . . 37

Structure and Modelling of the Three-Dimensional Boundary Layers on a

Rotating Disk . . . . . . . . . . . . . . . . . . . . . . . . . . 39

Stress and Stability Analysis of Surface Morphology of Elastic

and Piezoelectric Materials . . . . . . . . . . . . . . . . . . 39

3-D Experimental Fracture Analysis at High Temperatures . . . . . . . . 43

01-B

Film Cooling in a Pulsating Stream . . . . . . . . . . . . . . . 1 Ultrashort laser Heating and Phase Change in Liquids . . . . . . . . . . 4 Heat/Mass Transfer Enhancement in Separated and Vortex Flows . . . . . . . 25 Theory of Subcooled Boiling . . . . . . . . . . . . . . . . 30 Research on Combustion-Driven HVOF Thermal Sprays . . . . . . . . . 33 Critical Heat Flux in Micro-Channel Flow . . . . . . . . . . . . . . . 35 Coupled Particle Dispersion by Three-Dimensional Vortex Structures . . . . . 44

\section{1-C}

Hydrodynamic Instabilities and Coherent Structures . . . . . . . . . 1 Basic Studies of Transport Processes in Porous Media . . . . . . . . . . . 4 Wave Turbulence Interactions . . . . . . . . . . . . . . . 9 Gas and Solids Holdup in Three Phase Bioreactors ............. 11 


\section{1-C (cont'd)}

Experimental Studies of Reynolds Number Dependence of Turbulent Mixing

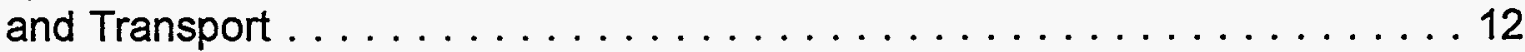

Two-Phase Potential Flow . . . . . . . . . . . . . . . . . . 12

Experimental and Analytical Investigations of Flows in Porous Media . . . . 13 Gas-Liquid Flow in Pipelines . . . . . . . . . . . . . . . . . 17

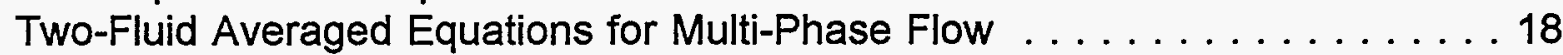

Turbulence Theory and Reduced Hydrodynamics . . . . . . . . . . . . 19

Contaminant Dispersal in Bounded Turbulent Shear Flows . . . . . . . . . . 21

Lubricated Transport of Viscous Materials . . . . . . . . . . . . . . 25

Microscopic Interfacial Phenomena During Flow in Porous Media . . . . . . . 31

Fundamental Study of Long-Short Interfacial Wave Interactions and Application to Flow Regime Development . . . . . . . . . . . . . . . . . . 32 Effect of Forced and Natural Convection on Solidification of Binary Mixtures . . 34 Interfacial Area and Interfacial Transfer in Two-Phase Flow . . . . . . . . . . 35 Development of Multidimensional Two-Fluid Modeling Capability . . . . . . 36 Development and Use of Image Scanning Ellipsometer to Study the Dynamics of Heated Thin Liquid Films . . . . . . . . . . . . . . . . . 36 Interfacial Area and Interfacial Transfer in Two-Phase Flow Systems . . . . . 44

\section{1-D}

Characterization of Metal Cutting Dynamics . . . . . . . . . . . . . . 20 Cryotribology (Low Temperature Friction and Wear): Development of Cryotribological Theories and Application to Cryogenic Devices . . . . . . 23

\section{3-A}

Modeling of Process Control . . . . . . . . . . . . . . . 8 Systematic Process Synthesis and Design Methods for Cost Effective Waste

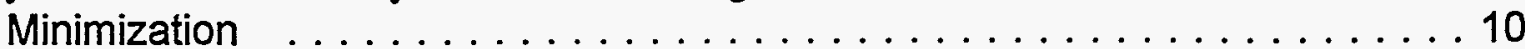
Application of Intelligent Control Systems to Mixed-Culture Bioprocesses . . . 15 Intelligent Control of Thermal Processes . . . . . . . . . . . . . . . . . 16 Model Building, Control and Optimization of Large Scale Systems . . . . . . 17 Synthesis and Optimization of Integrated Chemical Processes . . . . . . . 22 Partial Control of Complex Processing Systems . . . . . . . . . . . . . 29 Function-Based Biosensors for Use in Hazardous Waste Remediation . . . . . 39 Laser-Based Diagnostics for Plasma Chemistry . . . . . . . . . . . . . . . . . . 41 Visionics: An Integrated Approach to Analysis and Design of Intelligent Machines

New Process Modeling, Design and Control Strategies for Energy Efficiency, High Product Quality, and Improved Productivity in the Process Industries . . 45 
03-B

Nondestructive Evaluation of Superconductors $\ldots \ldots \ldots \ldots \ldots \ldots$

Two-Phase Flow Measurements by NMR . . . . . . . . . . . . 20

Pulse Propagation in Inhomogeneous Optical Waveguides . . . . . . . 21

Metal Transfer in Gas Metal Arc Welding . . . . . . . . . . . . . . . 22

Multivariable Control Of The Gas-Metal Arc Welding Process . . . . . . . . . 23

Thin-Film Characterization and Flaw Detection . . . . . . . . . . . . . 30

Optical Techniques for Characterization of High Temperature Superconductors 40

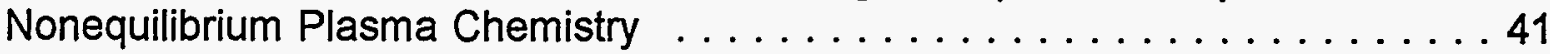

03-C

Center for Engineering Systems Advanced Research (CESAR) $\ldots \ldots \ldots \ldots 32$

06-A

Enzyme Adsorption and Activity at Liquid-Liquid Interfaces . . . . . . . . . . 3

Modeling of Thermal Plasma Processes . . . . . . . . . . . . . . 14

Fundamentals of Thermal Plasma Processing . . . . . . . . . . . . 14

Steady State and Transient Nucleation Kinetics . . . . . . . . . . . . . . . . 19

Development of Principles and Methodologies of Metabolic Engineering . . . . 24

Gelation of Dense Silica Suspensions: Effect of Shear . . . . . . . . . . . 27

Transport Properties of Disordered Porous Media From The Microstructure . . 34

06-B

Fundamental Studies of Spray Combustion . . . . . . . . . . . . 6

Mechanisms and Enhancements of Flame Stabilization ............ 33

Dynamically Active Scalars in Turbulent Combustion, Heat Transfer, and Geophysical Flows ....................... 38

06-C

Linear Kinetic Theory and Particle Transport in Stochastic Mixtures . . . . . . 5 Nonlinear Waves in Continuous Media: Application to Stochasticity and Energy Concentration .......................... 5 Broadband Signals: Signal Processing in Chaos . . . . . . . . . . 6 Noisy Nonlinear Systems . . . . . . . . . . . . . . . . . . 6 


\section{6-C (cont'd)}

Structure and Modeling of Turbulence $\ldots \ldots \ldots \ldots \ldots \ldots \ldots$

Spatiotemporal Wave Patterns: Models and Analysis . . . . . . . . . . 7

Experimental Study of 2D Traveling-Wave Patterns in Binary Fluid Convection 8 Bifurcations and Patterns in Nonlinear Dissipative Systems . . . . . . . . 8

Fundamentals and Techniques of Nonimaging Optics . . . . . . . . . 11

Theoretical and Computational Studies of Pattern Formation $\ldots \ldots \ldots \ldots 13$

Studies in Nonlinear Dynamics . . . . . . . . . . . . . . . . . . 19

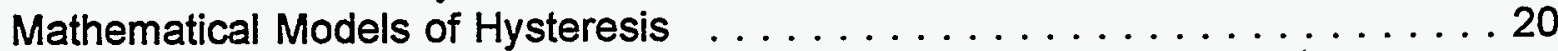

Macrostatistical Hydrodynamics . . . . . . . . . . . . . . . . 24

Thermal Plasma Chemical Vapor Deposition of Advanced Materials . . . . . . 26

Department of Energy Integrated Manufacturing Fellowship Program . . . . . 26

Center for Aerospace Research \& Education for Minority Students at

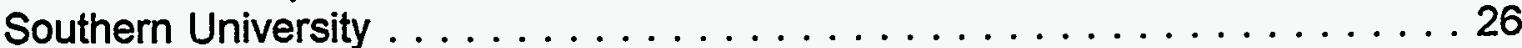

Industrial Liaison Pilot Program . . . . . . . . . . . . . . . . . 27

Development of Measurement Capabilities for the Thermophysical

Properties of Energy-Related Fluids . . . . . . . . . . . . . 28

High-Tc Superconductor-Semiconductor Integration and Contact Technology . 28

The Rheology of Concentrated Suspensions . . . . . . . . . . . . 28

Studies in Physico-Chemical Hydrodynamics of Extended Systems . . . . . . . 29

Sub-Electron Transfer of Electric Charge in Semiconductor Nanostructure . . . 30

Fragmentation and Dispersion of Powdered Solids in Viscous Liquids . . . . . . 31

Stability and Dynamics of Spatio-Temporal Structures . . . . . . . . . . . . 31

Flux Flow, Pinning, and Resistive Behavior in Superconducting Networks . . . 37

Direct and Inverse Problems in Statistical Wavefields . . . . . . . . . . 37

Some Basic Research Problems Related to Energy . . . . . . . . . . . . . . . 38

Topics in a Thermodynamic and Stochastic Theory of Nonlinear Processes

Far from Equilibrium . . . . . . . . . . . . . . . . . . 41

The Behavior of Matter Under Nonequilibrium Conditions: Fundamental

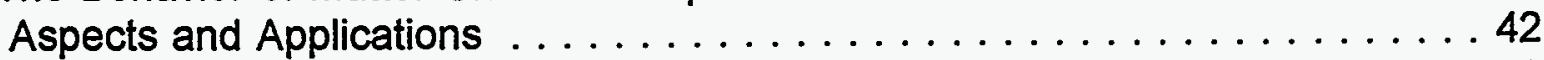

Complex Spatiotemporal Patterns in Nonequilibrium Systems . . . . . . . . . 42 


\section{Principal Investigators}

Investigator

H. Abarbanel

J. Achenbach

A. Acrivos

G. Ahlers

S. Altobellii

S. Atluri

S. Banerjee

S. Bankoff

J. Barhen

D. Barnett

P. Barton

T. Basar

R. Behringer

B. Berger

P. Bernard

L. Biegler

H. Blanch

I. Blankson

H. Brenner

F. Brust

D. Cannell

A. Caprihan

I. Catton

C. H. Chang

E.G.D. Cohen

S. Davis

D. Drew

T. Eagar

J. Eaton

J. Ekin

J. Epstein

L. Evans

H. Fasel

J. Fincke

A. Frenkel

L. Freund

E. Fukushima

H. Gao

J. Georgiadis

B. Ghosh

R. Goldstein

A. Graham

I. Grossmann

H.J.M. Hanley

T. Hanratty

D. Hardt
Phone: (619) 534-5590; Fax: (619) 534-7664; e-mail: hdia@hamilton.ucsd.edu . . . . . . . . 6 Phone: (847) 491-5527; Fax: (847) 491-5227; e-mail: achenbach@nwu.edu . . . . . . . . . 30 Phone: (212) 650-8159; Fax: (212) 650-6835; e-mail: acrivos@scisunsci.ccny.cuny.edu .... 28 Phone: (805) 893-3795; Fax: (805) 893-4170; e-mail: guenter@stc.ucsb.edu . . . . . . . . 8 Phone: (505) 262-7155; Fax: (505) 262-7043; e-mail: saltobelli@lucy.tli.org . . . . . . . . . 20 Phone: (404) 894-2758; Fax: (404) 894-2299; e-mail: atluri@cad.gatech.edu . . . . . . . . . 13 Phone: (805) 893-3456; Fax: (805) 893-4731; e-mail: banerjee@anemone.ucsb.edu ....... 9 Phone: (847) 491-5267; Fax: (847) 491-3728; e-mail: gbankoff@casbah.acns.nwu.edu . . . . . 30 Phone: (423) 574-7131; Fax: (423) 574-0405; e-mail: barhen@ornl.gov . . . . . . . . . . 32 Phone: (415) 723-4143; Fax: (415) 725-4034; e-mail: barnett@leland.stanford.edu ........ 39 Phone: (617) 253-6526; Fax: (617) 258-5042; e-mail: pib@mit.edu ............... 22 Phone: (217) 333-3607; Fax: (217) 244-1653; e-mail: tbasar@isaacs.csl.uiuc.edu . . . . . . 17 Phone: (919) 660-2550; Fax: (919) 660-2525; e-mail: bob@physics.phy.duke.edu . . . . . . 13 Phone: (301) 405-5283; Fax: (301) 314-9477; e-mail: berger@eng.umd.edu . . . . . . . . . . 20 Phone: (301) 405-5272; Fax: (301) 314-9477; e-mail: bernard@eng.umd.edu . . . . . . . . . . 21 Phone: (412) 268-2232; Fax: (412) 268-7139; e-mail: biegler@cmu.edu . . . . . . . . . . . 10 Phone: (510) 642-1387; Fax: (510) 643-1228; e-mail: blanch@socrates.berkeley.edu . . . . . 3 Phone: (216) 433-5823; Fax: (216) 433-3000; e-mail: isaiah.m.blankson@lerc.nasa.gov . . . 26 Phone: (617) 253-6687; Fax: (617) 258-8224; e-mail: hbrenner@mit.edu .............. 24 Phone: (614) 424-5034; Fax: (614) 424-3457; e-mail: brust@em.battelle.org . . . . . . . . 2 Phone: (805) 893-3150; Fax: (805) 893-4170; e-mail: david@alice.ucsb.edu . . . . . . . . . 8 Phone: (505) 262-7155; Fax: (505) 262-7043; e-mail: acaprihan@lucy.tli.org . . . . . . . . 20 Phone: (310) 825-5320; Fax: (310) 206-4830; e-mail: catton@.ucla.edu . . . . . . . . . . . 4 Phone: (208) 526-2886; Fax: (208) 526-0528; e-mail: chc@inel.gov . . . . . . . . . . . 14 -Phone: (212) 327-8855: Fax: (212) 327-8507; e-mail: egdc@rockvax.rockefeller.edu ..... 38 Phone: (847) 491-5397; Fax: (847) 491-2178; e-mail: sdavis@casbah.acns.nwu.edu ..... 30 Phone: (518) 276-6903; Fax: (518) 276-4824; e-mail: drewd@rpi.edu . . . . . . . . . . . 36 Phone: (617) 253-0948; Fax: (617) 252-1773; e-mail: tweagar@mit.edu . . . . . . . . . . 22

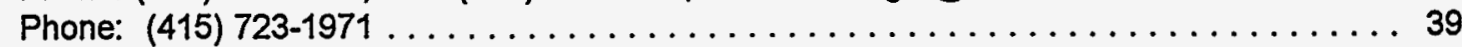
Phone: (303) 497-5448; Fax: (303) 497-5316; e-mail: ekin@boulder.nist.gov .......... 28 Phone: (208) 526-6874; Fax: (208) 526-0690; e-mail: esj@inel.gov . . . . . . . . . . . . . . . 15 Phone: (617) 577-0100; Fax: (617) 577-0722; e-mail: larry.evans@aspentech.com . . . . . . 22 Phone: (520) 621-2771; Fax: (520) 621-8191; e-mail: faselh@ccit.arizona.edu .......... 1 Phone: (208) 526-2031; Fax: (208) 526-2814; e-mail: jf1@inel.gov. . . . . . . . . . . . . 14 Phone: (205) 348-5434; Fax: (205) 348-7067; e-mail: afrenkel@gp.as.ua.edu . . . . . . . . 1 Phone: (401) 863-1476; Fax: (401) 863-1157; e-mail: freund@isaac.engin.brown.edu ...... 3 Phone: (505) 262-7155; Fax: (505) 262-7043; e-mail: eilchi@lucy.tli.org .............. 20 Phone: (415) 725-2560; Fax: (415) 723-1778; e-mail: gao@am-sun2.stanford.edu . . . . . . 39 Phone: (217) 244-7578; Fax: (217) 333-1942; e-mail: georgia@staff.uiuc.edu . . . . . . . 13 Phone: (314) 935-6039; Fax: (314) 935-6121; e-mail: ghosh@zach.wustl.edu . . . . . . . . 43 Phone: (612) 625-5552; Fax: (612) 625-3434; e-mail: rjgumn@mailbox.mail.umn.edu . . . . . 25 Phone: (505) 665-5259: Fax: (505) 665-9521; e-mail: graham@lanl.gov . . . . . . . . . . . 24 Phone: (412) 268-2228; Fax: (412) 268-7139; e-mail: grossmann@cmu.edu . . . . . . . . 10 Phone: (303) 497-3320; Fax: (303) 497-5044; e-mail: hanley@central.bldrdoc.gov . . . . . . 27 Phone: (217) 333-1318; Fax: (217) 333-5052; e-mail: thanratt@uiuc.edu . . . . . . . . . . 17 Phone: (617) 253-2252; Fax: (617) 252-1399; e-mail: hardt@mit.edu .............. 23 


\section{Principal Investigators (cont'd - pg. 2)}

W. Haynes

J. Heberlein

J. Hickman

F. Incropera

M. Ishii

Y. IWasa

G. Johnson

J. Johnson

D. Joseph

A. Kaufman

R. Kayser

A. Kerstein

G. Kino

A. Kobayashi

G. Kojasoy

R. Kraichnan

D. Krajcinovic

E. Krempl

C. Kruger

R. Lahey

J. Lang

C. Law

P. Libby

K. Likharev

K. Lindenberg

R. Littlejohn

W. Lloyd

F. McClintock

M. McCready

J. McLaughlin

R. Mann

J. Mayergoyz

C. Menyuk

M. Miksis

I. Minis

L. Mondy

F. Moon

J. Moreland

I. Mudawar

E. Novikov

E. Oblow

L. Ong

A. Ortega

J. Ottino

T. Owano

L. Parker

D. Parks

T. Petrosky
Phone: (303) 497-3247; Fax: (303) 497-5044; e-mail: william.haynes@nist.gov . . . . . . . 28 Phone: (612) 625-4538; Fax: (612) 624-1398; e-mail: jvrh@me.umn.edu . . . . . . . . . 26 Phone: (301) 309-1125; Fax: (301) 309-8499; e-mail: james.j.hickman@cpmx.saic.com ... . 39 Phone: (317) 494-5688; Fax: (317) 494-0539; e-mail: fpi@ecn.purdue.edu . . . . . . . . . . 34 Phone: (765) 494-4587; Fax: (765) 494-9570; e-mail: grad@ecn.purdue.edu . . . . . . . . . 35 Phone: (617) 253-5548; Fax: (617) 253-5405; e-mail: iwasa@jokaku.pfc.mit.edu . . . . . . 23 Phone: (919) 684-7754; Fax: (919) 684-7122; e-mail: gaj@orion.mc.duke.edu . . . . . . . . 13 Phone: (208) 526-9021; Fax: (208) 526-0690; e-mail: jsq@inel.gov . . . . . . . . . . . . . 15 Phone: (612) 625-0309; Fax: (612) 626-1558; e-mail: joseph@aem.umn.edu . . . . . . . . . 25 Phone: (510) 486-7899; Fax: (510) 486-7550; e-mail: ankaufman@lbl.gov . . . . . . . . . 19 Phone: (301) 975-2483; Fax: (301) 869-4020; e-mail: richard.kayser@nist.gov . . . . . . . . 28 Phone: (510) 294-2390; Fax: (510) 294-1004; e-mail: kerstein@ca.sandia.gov . . . . . . . . . 38 Phone: (415) 723-0205; Fax: (415) 725-2533; e-mail: kino@ee.stanford.edu . . . . . . . . . 40 Phone: (206) 543-5488; Fax: (206) 685-8047; e-mail: ask@u.washington.edu . . . . . . . . 43 Phone: (414) 229-5639; Fax: (414) 229-6958; e-mail: kojasoy@csd.uwm.edu . . . . . . . . . . 44 Phone: (505) 986-3979; Fax: (505) 989-4737; e-mail: rhk@lanl.gov ................ 19 Phone: (602) 965-8656; Fax: (602) 965-1384; e-mail: krajcino@asuvax.eas.asu.edu . . . . . . 2 Phone: (518) 276-6985; Fax: (518) 276-6025; e-mail: krempe@rpi.edu . . . . . . . . . . . . 35 Phone: (415) 723-0977; Fax: (415) 725-1653; e-mail: kruger@soe.stanford.edu . . . . . . . . 41 Phone: (518) 276-6298; Fax: (518) 276-8788; e-mail: laheyr@rpi.edu . . . . . . . . . . . 36 Phone: (617) 253-4687; Fax: (617) 258-6774; e-mail: lang@mit.edu . . . . . . . . . . . 22 Phone: (609) 258-5271; Fax: (609) 258-6233; e-mail: cklaw@princeton.edu . . . . . . . . . 33 Phone: (619) 534-3168; Fax: (619) 534-5354; e-mail: libby@ames.ucsd.edu . . . . . . . . . 6 Phone: (516) 632-8159; Fax: (516) 632-8774; e-mail: klikharev@ccmail.sunysb.edu . . . . . . 30 Phone: (619) 534-3285; Fax: (619) 534-7244; e-mail: klindenberg@ucsd.edu . . . . . . . . . 6 Phone: (510) 486-7901; Fax: (510) 486-7550; e-mail: rglittlejohn@lbl.gov . . . . . . . . . . 19 Phone: (208) 526-0808; Fax: (208) 526-0690; e-mail: qri@inel.gov . . . . . . . . . . . . . 15 Phone: (617) 253-2219; Fax: (617) 258-8742 . . . . . . . . . . . . . . . . . 23 Phone: (219) 631-7146; Fax: (219) 631-8366; e-mail: mccready.1@nd.edu . . . . . . . . 32 Phone: (315) 268-6663; Fax: (315) 268-6654; e-mail: jmclau@sun.soe.clarkson.edu . . . . . . 11 Phone: (423) 574-5845; Fax: (423) 574-7860 . . . . . . . . . . . . . . . . . 32 Phone: (301) 405-3657; Fax: (301) 314-9281; e-mail: isaak@eng.umd.edu . . . . . . . . 20 Phone: (410) 455-3501; Fax: (410) 455-6500; e-mail: menyuk@umbc.edu . . . . . . . . . . . 21 Phone: (847) 491-5585: Fax: (847) 491-2178; e-mail: miksis@nwu.edu . . . . . . . . . . . 31 Phone: (301) 405-5310: Fax: (301) 314-9477; e-mail: minis@isr.umd.edu . . . . . . . . . 20 Phone: (505) 844-1755; Fax: (505) 844-8251; e-mail: lamondy@sandia.gov . . . . . . . . . . 24 Phone: (607) 255-7146; Fax: (607) 255-1222; e-mail: fcm3@cornell.edu .............. 11 Phone: (303) 497-3641; Fax: (303) 497-5316; e-mail: moreland@boulder.nist.gov . . . . . . 28 Phone: (765) 494-5705; Fax: (765) 494-0539; e-mail: mudawar@ecn.purdue.edu . . . . . . . . 35 Phone: (619) 534-0816; Fax: (619) 534-7664; e-mail: enovikov@ucsd.edu . . . . . . . . . . 7 Phone: (423) 574-6187; Fax: (423) 574-7860; e-mail: oblowem@ornl.gov . . . . . . . . . . 32 Phone: (301) 405-5343; Fax: (301) 314-9477; e-mail: lawrence@eng.umd.edu ........... 21 Phone: (520) 621-6787; Fax: (520) 621-8191; e-mail: ortega@ccit.arizona.edu . . . . . . . . . 1 Phone: (847) 491-3558; Fax: (847) 491-3728; e-mail: ottino@chem-eng.nwu.edu . . . . . . . 31 Phone: (415) 723-1295; Fax: (415) 723-1748; e-mail: owano@saha.stanford.edu . . . . . . 41 Phone: (423) 241-4959; Fax: (423) 574-7860; e-mail: parkerle@ornl.gov . . . . . . . . . 32 Phone: (617) 253-0033; Fax: (617) 258-8742 . . . . . . . . . . . . . . . . . . . 23 Phone: (512) 471-7253; Fax: (512) 471-9621; e-mail: petrosky@physics.utexas.edu . . . . . . 42 


\section{Principal Investigators (cont'd - pg. 3)}

J. Plawsky

G. Pomraning

I. Prigogine

A. Prosperetti

V. Protopopescu

S. Putterman

M. Rabinovich

E. Rabinowicz

N.S.V. Rao

W. Ray

D. Reister

W. Reuter

H. Riecke

I. Rinard

A. Rosakis

J. Ross

T. Rozzell

$H$. Sehitoglu

G. Settles

J. Sheridan

R. Shinnar

G. Sivashinsky

H. Smartt

R. Spanos

G. Stephanopoulos

D. Stoner

C. Surko

H. Swinney

S. Teitel

K. Telschow

C. Tien

S. Torquato

T. Troutt

L. Tsimring

J. Viñals

J. Wallace

G. Wallis

Z. Warhaft

P.C. Wayner

A. Westerberg

G. Wilemski

F. Williams

R. Winston

E. Wolf

I. Wygnanski

R. Zare
Phone: (518) 276-6049; Fax: (518) 276-4030; e-mail: plawsky@rpi.edu . . . . . . . . . . . . . . 36 Phone: (310) 825-1744; Fax: (310) 206-2302; e-mail: pom@seas.ucla.edu . . . . . . . . . . . . 5 Phone: (512) 471-7253; Fax: (512) 471-9621; e-mail: annie@physics.utexas.edu .......... 42 Phone: (410) 516-8534; Fax: (410) 516-7254; e-mail: prosper@titan.me.jhu.edu . . . . . . . . 18 Phone: (423) 574-4722; Fax: (423) 574-0405; e-mail: protopopesva@ornl.gov . . . . . . . . 32 Phone: (310) 825-2269; Fax: (310) 206-5668; e-mail: putterman@physics.ucla.edu ........ 5 Phone: (619) 534-0816; Fax: (619) 534-7664; e-mail: lev@gibbs.ucsd.edu ............... 7

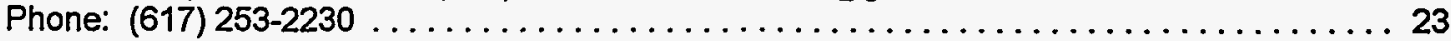
Phone: (423) 574-7517; Fax: (423) 574-7860; e-mail: raons@ornl.gov ............... 32 Phone: (608) 263-4732; Fax: (608) 262-0832; e-mail: ray@engr.wisc.edu . . . . . . . . . . . . 45 Phone: (423) 574-2272; Fax: (423) 574-7860; e-mail: dbr@ornl.gov.................... 32 Phone: (208) 526-1708; Fax: (208) 526-0690; e-mail: wgr2@inel.gov ................ 15 Phone: (847) 491-3345; Fax: (847) 491-2178; e-mail: h-riecke@nwu.edu . . . . . . . . . . . 31 Phone: (212) 650-7135; Fax: (212) 650-6686; e-mail: rinard@che-mail.engr.ccny.cuny.edu ... 29 Phone: (818) 395-4523; Fax: (818) 304-0175; e-mail: rosakis@atlantis.caltech.edu .......... 10 Phone: (415) 723-9203; Fax: (415) 723-4817; e-mail: ross@chemistry.stanford.edu ........ 42 Phone: (202) 334-2908; Fax: (202) 334-3419; e-mail: trozzell@nas.edu . . . . . . . . . . . . 26 Phone: (217) 333-4112; Fax: (217) 244-6534; e-mail: huseyin@ux1.cso.uiuc.edu ........... 18 Phone: (814) 863-1504; Fax: (814) 865-0118; e-mail: gss2@psu.edu . . . . . . . . . . . . . 33 Phone: (313) 995-4963; Fax. (313) 995-1150; e-mail: johns@ncms.org . . . . . . . . . . 27 Phone: (212) 650-6679; Fax: (212) 650-6686; e-mail: shinnar@che-mail.engr.ccny.cuny.edu .. 29 Phone: (212) 650-8157; Fax: (212) 650-6835 . . . . . . . . . . . . . . . . . . 29 Phone: (208) 526-8333; Fax: (208) 526-0690; e-mail: hbs@inel.gov................. 16 Phone: (713) 527-4909; Fax: (713) 285-5191; e-mail: spanos@rice.edu . . . . . . . . . . 37 Phone: (617) 253-4583; Fax: (617) 253-3122; e-mail: gregstep@mit.edu . . . . . . . . . . . . 24 Phone: (208) 526-8786; Fax: (208) 526-0828; e-mail: dls2@inel.gov ................. 15 Phone: (619) 534-6880; Fax: (619) 534-0173; e-mail: csurko@ucsd.edu ............... 8 Phone: (512)-471-4619; Fax: (512)-471-1558; e-mail-swinney@chaos.ph.utexas.edu........ 42 Phone: (716) 275-4039; Fax: (716) 275-8527; e-mail: stte@pas.rochester.edu . . . . . . . . . 37 Phone: (208) 526-1264; Fax: (208) 526-0690; e-mail: telsch@inel.gov . . .............. 16 Phone: (510) 642-7464; Fax: (510) 643-5499; e-mail: joycedev@uclink4.berkeley.edu ....... 4 Phone: (609) 258-3341; Fax: (609) 258-2685; e-mail: torquato@matter.princeton.edu ....... 34 Phone: (509) 335-4375; Fax: (509) 335-4662; e-mail: troutt@mme.wsu.edu .............. 44 Phone: (619) 534-0816; Fax: (619) 534-7664; e-mail: lev@gibbs.ucsd.edu ............. 8 Phone: (904) 644-1010; Fax: (904) 644-0098; e-mail: vinals@scri.fsu.edu .............. 13 Phone: (301) 405-5271; Fax: (301) 314-9477; e-mail: wallace@eng.umd.edu ............. 21 Phone: (603)646-2789; Fax: (603) 646-3856; e-mail: graham.b.wallis@dartmouth.edu ...... 12 Phone: (607) 255-3898; Fax: (607) 255-1222; e-mail: zw16@cornell.edu .............. 12 Phone: (518) 276-6199; Fax: (518) 276-4030; e-mail: wayner@rpi.edu ................ 36 Phone: (412) 268-2344; Fax: (412) 268-7139; e-mail: a.westerberg@cmu.edu ........... 10 Phone: (510) 422-7919; Fax: (510) 422.4982; e-mail: wilemski1@llnl.gov . . . . . . . . . . 19 Phone: (619) 534-5492; Fax: (619) 534-5354; e-mail: faw@ames.ucsd.edu...............6 6 Phone: (312) 702-7756; Fax: (312) 702-6317; e-mail: winston@rainbow.uchicago.edu ........ 11 Phone: $(716)$ 275-4397: Fax: $(716)$ 473-0687 ............................ 37 Phone: (520) 621-6089; Fax: (520) 621-8191; e-mail: wygy@bigdog.engr.arizona.edu ...... 1 Phone: (650) 723-3062; Fax: (650) 723-9262; e-mail: zare@stanford.edu ............... 41 